\title{
BASIS IN AN INVARIANT SPACE OF ENTIRE FUNCTIONS
}

\author{
A. S. KRIVOSHEEV AND O. A. KRIVOSHEEVA
}

\begin{abstract}
The existence of a basis is studied in a space of entire functions invariant under the differentiation operator. It is proved that every such space possesses a basis consisting of linear combinations of generalized eigenvectors. These linear combinations are formed within groups of exponents of arbitrarily small relative diameter. A complete description of the way to split the exponents into groups is obtained. Also, a criterion is found for the existence of a basis constructed by groups of zero relative diameter (so-called relatively small groups). In this connection a new criterion is obtained for the finiteness of the lower indicator of an entire function of exponential type.
\end{abstract}

\section{$\S 1$. INTRODUCTION}

Let $H(\mathbb{C})$ denote the space of entire functions with the topology of uniform convergence on compact sets, and let $\mathcal{W}$ be a nontrivial $(\mathcal{W} \neq H(\mathbb{C}),\{0\})$ subspace invariant under differentiation, i.e., containing the derivatives of all functions that belong to it. Spaces of solutions of homogeneous convolution equations and their systems are such, the homogeneous convolution equation being an equation of the form $\mu(g(z+w)) \equiv 0$, $g \in H(\mathbb{C})$, where $\mu$ is an element of the strong dual $H^{*}(\mathbb{C})$, called an analytic functional. Moreover, every subspace of $H(\mathbb{C})$ invariant under differentiation is the space of solutions of a system of at most two such equations (see, e.g., [1, Chapter II, $\S 7$, Theorem 7.5]).

Let $\Lambda=\left\{\lambda_{k}, n_{k}\right\}$ be the collection of all points of the spectrum and their multiplicities for the differentiation operator on $\mathcal{W}$. If $\mathcal{W}$ is nontrivial, there is a functional $\mu \neq 0$ that annihilates $\mathcal{W}$. Its Laplace transform $f(\lambda)=\mu(\exp \lambda z)$ is an entire function (see, e.g., [2. Chapter III, §12]), which, as can easily be realized, vanishes at the points $\lambda_{k}$ with multiplicity of an least $n_{k}$. Since the exponentials are dense in $H(\mathbb{C})$, we see that $f \not \equiv 0$. Thus, there are at most countably many points $\lambda_{k}$, and their multiplicities are finite. If the set of these points is countable, it accumulates only towards $\infty$. Note that the Laplace transformation establishes an isomorphism between $H^{*}(\mathbb{C})$ and the space of entire functions of exponential type (see, e.g., [2, Chapter III, §12, Theorem 12.3]). If $\mathcal{W}$ is the space of solutions of the convolution equation $\mu(g(z+w)) \equiv 0$, then $\Lambda$ coincides with the collection of zeros and their multiplicities for the Laplace transform of $\mu$ (this Laplace transform is called the characteristic function of the above convolution operator).

As examples of convolution equations, we can name linear differential, difference, and difference-differential equations of finite or infinite order and with constant coefficients, and also certain integral equations. If the spectrum of a space $\mathcal{W}$ is finite, then $\mathcal{W}$ coincides with the space of solutions of a linear homogeneous differential equation of finite order and with constant coefficients.

Let $\mathcal{E}(\Lambda)=\left\{z^{n} \exp \left(\lambda_{k} z\right)\right\}, k \geq 1, n=0, \ldots, n_{k}-1$, be the system of generalized eigenvectors for the differentiation operator on $\mathcal{W}$. It is natural to look for ways of representing all functions in $\mathcal{W}$ in terms of elements of $\mathcal{E}$. The first result in the framework

2010 Mathematics Subject Classification. Primary 30D10.

Key words and phrases. Entire function, basis, invariant subspace, interpolation. 
of this problem was Euler's fundamental principle for the spaces of solutions of linear homogeneous differential equations of finite order with constant coefficients. Every such solution can be written as a finite sum of the form $\sum z^{n} \exp \left(\lambda_{k} z\right)$, where the $\lambda_{k}$ are the roots of the characteristic polynomial whose multiplicities are $n_{k}$, and $n$ may vary from 0 to $n_{k}-1$. In the case where the spectrum of $\mathcal{W}$ is infinite, the representation problem becomes much more involved. Ideally, it would be desirable to represent a function $g \in \mathcal{W}$ as a series

$$
g(z)=\sum_{k=1, n=0}^{\infty, n_{k}-1} d_{k, n} z^{n} \exp \left(\lambda_{k} z\right)
$$

convergent uniformly on every compact subset of the plane. If every function $g \in \mathcal{W}$ is represented by a series (1.1), we say that the fundamental principle is fulfilled in $\mathcal{W}$. It should be noted that, whenever it exists, an expansion (1.1) is unique for every nontrivial subspace $\mathcal{W}$. Indeed (see, e.g., [3, Chapter I, §1, item 3]), in this case the system $\mathcal{E}(\Lambda)$ always admits a biorthogonal system of functionals $\left\{\mu_{k, n}\right\}_{k=1, n=0}^{\infty, n_{k}-1} \subset H^{*}(\mathbb{C})$ (i.e., $\mu_{k, n}\left(z^{l} \exp \left(\lambda_{j} z\right)\right)=1$ if $k=j, n=l$, and $\mu_{k, n}\left(z^{l} \exp \left(\lambda_{j} z\right)\right)=0$ otherwise). Then, clearly, $d_{k, n}=\mu_{k, n}(g), l \geq 1, n=0, \ldots, n_{k}-1$.

The first step towards the representation (1.1) is solution of the spectral synthesis problem, i.e., finding conditions under which the system $\mathcal{E}(\Lambda)$ is complete in $\mathcal{W}$. In 1947, L. Schwartz proved that an arbitrary closed invariant subspace $\mathcal{W} \subset H(\mathbb{C})$ admits spectral synthesis. Thus, $\mathcal{W}$ is uniquely determined by its multiple spectrum $\Lambda=\left\{\lambda_{k}, n_{k}\right\}$ as the closed linear span of the system $\mathcal{E}(\Lambda)$.

On the other hand, the subspace of $H(\mathbb{C})$ equal to the closure of the linear span of some system $\mathcal{E}(\Lambda)$ is closed and invariant under differentiation. In what follows, this subspace will be denoted by $\mathcal{W}(\Lambda)$ and will be called the subspace generated by $\Lambda$.

In 1951, A. O. Gelfond refined Schwartz's result in the case where $\mathcal{W}$ is the space of solutions of the convolution equation $\mu(g(z+w)) \equiv 0$. He showed that every function $g \in \mathcal{W}$ is representable by a series (1.1) "with brackets"

$$
g(z)=\sum_{m=1}^{\infty}\left(\sum_{\lambda_{k} \in U_{m}} \sum_{n=0}^{n_{k}-1} d_{k, n} z^{n} \exp \left(\lambda_{k} z\right)\right)
$$

that converges uniformly on compact subsets of $\mathbb{C}$. Here $U_{m}$ is the group of zeros $\lambda_{k}$ (with multiplicities $n_{k}$ ) for the characteristic function $f$ of the convolution operator that belong to the annulus $r_{m} \leq|\lambda|<r_{m+1}$, and $\left\{r_{m}\right\}$ is a monotone increasing sequence that tends to infinity and has the property that $\ln |f(\lambda)| \geq-a|\lambda|,|\lambda|=r_{m}, m \geq 1$, with some constant $a>0$. A. F. Leont'ev (see [1, Chapter II, §7, Theorem 7.2]) extended this result to arbitrary nontrivial invariant spaces of entire functions. Specifically, the role of $f$ (as far as we deal with lower estimates on circles, i.e., when we split $\Lambda$ into the groups $U_{m}$ ) can be played by an arbitrary entire function of exponential type that has a zero of multiplicity of at most $n_{k}$ at each $\lambda_{k}$.

Only the nontriviality of $\mathcal{W}(\Lambda)$ is required for the representation (1.2). Invoking the Laplace transformation, we see that this space is nontrivial if and only if there exists an entire function of exponential type that has a zero of multiplicity of at least $n_{k}$ at each $\lambda_{k}$. The Lindelöf theorem shows (see [1, Chapter I, §3, Theorem 3.9]) that such an $f$ exists if and only if the upper density of $\Lambda$ is finite. For example, such a function can be chosen as follows:

$$
L(\lambda)=\prod_{k=1}^{\infty}\left(1-\frac{\lambda^{2}}{\lambda_{k}^{2}}\right)^{n_{k}}, \quad \lambda \in \mathbb{C} .
$$


In distinction with (1.2), the representation (1.1) cannot be ensured by the mere finiteness of the upper density of $\Lambda$. There is an example of a series (1.2) convergent on $\mathbb{C}$ and such that the corresponding series (1.1) diverges everywhere. This is achieved when the $\lambda_{k}$ strongly approach one another near infinity; a similar example will be presented in $\S 8$ in a more general situation. Thus, for the representation (1.1) we must impose some "separation" condition on the points $\lambda_{k}$. For this, A. F. Leont'ev employed Bernstein's condensation index, extending its definition to complex sequences $\Lambda$ with simple (nonmultiple) $\lambda_{k}$ :

$$
\gamma(\Lambda)=\limsup _{k \rightarrow \infty} \frac{1}{\left|\lambda_{k}\right|} \ln \left|\frac{1}{L^{\prime}\left(\lambda_{k}\right)}\right|
$$

where $L$ is given by (1.3) with $n_{k}=1$. In this way, Leont'ev gave a criterion for the fundamental principle for invariant subspaces $\mathcal{W}(\Lambda) \subset H(\mathbb{C})$ in the case of simple $\lambda_{k}$, see [3, Chapter IV, $\S 2$, Theorem 4.2.12]). Specifically, every $g \in \mathcal{W}(\Lambda)$ is representable by a series (1.1) if and only if the indices $\gamma\left(\Lambda_{i}\right), i=1,2,3$, are finite; these indices are defined in terms of the functions $L_{i}(\lambda), i=1,2,3$, each of which corresponds to the points $\lambda_{k}$ that belong to one of three angles that cover the plane. Also, Leont'ev gave a simpler criterion for the fundamental principle in spaces of entire solutions of homogeneous convolution equations with simple $\lambda_{k}$ (see [3, Chapter IV, $\S 2$, Theorems 4.2.4-4.2.6]). Each of these solutions is representable by a series (1.1) if and only if the index $\gamma(\Lambda)$ defined in terms of the characteristic function $L(\lambda)$ for the convolution operator is finite.

In these results, the index $\gamma(\Lambda)$ for complex sequences is not uniquely defined. Also, it is of global nature because it arises as a limit of quantities whose calculation requires all $\lambda_{k}$ 's. It might be supposed that the local behavior of the $\lambda_{k}$ (their mutual separation) is influenced by their distribution on the plane. However, this is not so. In order to confirm this, a new condensation index $\mathcal{S}_{\Lambda}$ of sequences $\Lambda$ was introduced in [6]. It is defined as a limit of certain quantities depending on the points $\lambda_{j}$ that belong to an arbitrarily small (relative to $\left|\lambda_{k}\right|$ ) neighborhood of $\lambda_{k}$; see $\S 2$ for the definition of $\mathcal{S}_{\Lambda}$. In that paper, a criterion for the fundamental principle was found for arbitrary invariant subspaces of functions analytic in a convex domain on the complex plane. In particular, it was proved there that a representation (1.1) is possible for every $g \in \mathcal{W}(\Lambda) \subset H(\mathbb{C})$ if and only if $\mathcal{S}_{\Lambda}$ is finite.

It should be noted that the fundamental principle is equivalent to the solvability of a multiple interpolation problem in the space of entire functions of exponential type with the $\lambda_{k}$ in the role of interpolation nodes (see, e.g., [6, Proposition 2.10]). Largely, this relationship was established due to Leont'ev's work.

Thus, presently, the conditions of representability by series (1.1) for arbitrary invariant subspaces of entire functions are studied fully. But the situation when $\mathcal{S}_{\Lambda}=-\infty$ is still not quite clear. In this case, (1.1) is impossible. But (1.2) always occurs. In this connection, it is natural to ask about representations "intermediate" between (1.1) and (1.2). Is it possible to replace (1.2) (which is a series (1.1) "with brackets") by a "pure" series whose terms are linear combinations of functions belonging to $\mathcal{E}(\Lambda)$ with exponents within groups $U_{m}$ in such a way that these linear combinations be one and the same for all $g \in \mathcal{W}(\Lambda)$ ? In other words, does $\mathcal{W}(\Lambda)$ possess a basis consisting of linear combinations of generalized eigenvectors for the differentiation operator and constructed in accordance with a certain splitting $U=\left\{U_{m}\right\}$ of the sequence $\Lambda$ into groups? If such a basis exists, many other questions arise. How to find a partition $U$ and how to describe all suitable partitions? How to form linear combinations within a group and how to describe all suitable linear combinations? How small the diameter of $U_{m}$ can be made, i.e., to what extent the new "pure" series shares the properties of (1.1)? Finally, how to 
describe the space of coefficients of convergent series with respect to this basis? In other words, what space of numerical sequences is identifiable with $\mathcal{W}(\Lambda)$ ?

The present paper is devoted to answers to these questions. It consists of 9 sections. In $\S 2$, we study numerical characteristics of a complex sequence. In particular, for an arbitrary partition $U=\left\{U_{m}\right\}$ of the sequence $\Lambda$, we introduce a quantity $\mathcal{S}_{\Lambda}(U)$, which measures the asymptotic closeness of the groups $U_{m}$ to one another. In the sequel, this characteristic will allow us to describe all partitions suitable for constructing a basis. Some properties of functions involved in the definition of $\mathcal{S}_{\Lambda}(U)$ are studied. In Theorem 2.1, it is proved that the upper density of $\Lambda$ is finite if and only if $U$ can be split into groups $U_{m}$ with a prescribed asymptotic smallness (relative to the moduli of $\lambda_{k} \in U_{m}$ ) of the diameters.

In $\S 3$, we study splittings into the so-called relatively small groups $U_{m}$, i.e., into groups whose diameters and cardinalities are infinitesimal as $m \rightarrow \infty$ relative to the moduli of $\lambda_{k} \in U_{m}$. A characteristic $S_{\Lambda}^{1}$ is introduced, which leads to a criterion (namely, $S_{\Lambda}^{1}>-\infty$, Theorem 3.2) for the existence of a suitable splitting $U$ of $\Lambda$ into relatively small groups. Some properties of this characteristic are studied (Theorem 3.1). It has turned out that, if $\Lambda$ is the zero set (with multiplicities) for an entire function of exponential type, the finiteness of $S_{\Lambda}^{1}$ is equivalent to the finiteness of the lower indicator of this function. This fact will be established in $\S 6$ (Theorem 6.1). In distinction to an earlier finiteness criterion for the lower indicator (see [7]), the new criterion is of local nature. Next, we want to strongly emphasize its geometric essence.

$\S \S 4$ and 5 are auxiliary. The results proved there will be used in $\S 7$ for the solution of an interpolation problem (Theorem 7.1) required for the construction of a basis in $\mathcal{W}(\Lambda)$. In Theorem 4.1, a complex sequence is extended to the zero set of an entire function of exponential type in a special way, which makes it possible to retain the required separation of groups, whatever be the partition of the initial sequence. Also, certain lower estimates for functions of exponential type are obtained (Theorem 5.1).

In $\S 8$, we construct a function in $\mathcal{W}(\Lambda)$ that does not expand in a series (1.2) at any point of the plane provided the groups $U_{m}$ strongly approach one another as $m \rightarrow \infty$.

The principal results are collected in the last section. Given a partition $U=\left\{U_{m}\right\}$ of the sequence in question, we construct a special system $\mathcal{E}(\Lambda, U)$ of exponential polynomials that is intimately related to the interpolation problem mentioned above. On the basis of the solution of that problem, we shall show that, under the condition $\mathcal{S}_{\Lambda}(U)>-\infty$, the system $\mathcal{E}(\Lambda, U)$ is a Köthe basis in $\mathcal{W}(\Lambda)$. Moreover, the space of coefficients of expansions with respect to this basis will be described completely (Theorem 9.1). In Theorem 9.2, with the help of Theorem 8.1 we shall prove that the condition $\mathcal{S}_{\Lambda}(U)>-\infty$ is necessary for the existence of a basis constructed by the partition $U$. These results imply that, being a basis, the system $\mathcal{E}(\Lambda, U)$ turns out automatically to be a Köthe basis. They also imply that the above condition is a criterion for $\mathcal{E}(\Lambda, U)$ to be a basis (Theorem 9.5) and that the existence of a basis in $\mathcal{W}(\Lambda)$ implies that $\mathcal{E}(\Lambda, U)$ is a basis (Theorem 9.4). In particular, Theorem 9.5 covers the solution of the fundamental principle problem for an invariant space of entire functions, found in [6, Theorem 5.1].

The section is finished with the study of other bases (different from $\mathcal{E}(\Lambda, U)$ ) in $\mathcal{W}(\Lambda)$ that consist of linear combinations of generalized eigenvectors of the differential operator such that the exponents of each linear combination are within one of the groups $U_{m}$. In Theorem 9.6 all possible bases are constructed under the condition $\mathcal{S}_{\Lambda}(U)>-\infty$. By Theorem 9.2, there is no such bases if this condition is violated. All such bases are Köthe bases automatically. Also, the space of expansion coefficients is described. By using Theorem 2.1, it is shown that a nontrivial subspace $\mathcal{W}(\Lambda)$ always possesses a basis constructed by groups of arbitrarily small relative diameter (Theorem 9.7). Moreover, 
with the help of Theorem 3.2, in Theorem 9.8 we give a criterion for the existence of a basis with respect to groups of zero relative diameter (i.e., with respect to relatively small groups). In the last result of the paper (Theorem 9.9), we study the case where $\mathcal{W}(\Lambda)$ is the space of solutions of a homogeneous convolution equation. Here, with the help of Theorem 6.1, we manage to obtain yet another criterion of the existence of a basis with respect to relatively small groups, namely, the boundedness of the lower indicator for the characteristic function of the convolution operator.

To conclude the Introduction, we note that the existence problem for a basis with respect to relatively small groups in an invariant subspace was studied by the authors in [8]. In distinction with the present paper, in [8] we treated invariant spaces of functions analytic in a bounded convex domain. A criterion was found for the existence of such a basis in classical terms of the regular growth of a certain entire function of exponential type that vanishes at each $\lambda_{k}$ with multiplicity of at least $n_{k}$. A drawback of this criterion is an "intermediator" (an entire function) involved in the statement. In the present paper, for invariant spaces of entire functions, we give a criterion stated exclusively in terms of the geometry of the sequence $\Lambda=\left\{\lambda_{k}, n_{k}\right\}_{k=1}^{\infty}$ (i.e., the finiteness of the quantity $S_{\Lambda}^{1}$ ). Next, a principal difference of the case considered here from the case of a bounded convex domain lies in the fact that an invariant space of entire functions always possesses a basis (Theorem 9.7) with respect to groups of arbitrarily small relative diameter (but not with respect to relatively small groups).

\section{$\S 2$. Characteristics of a COMplex Sequence}

In this section, we study splittings of the sequence $\Lambda$ that are suitable for constructing a basis in $\mathcal{W}(\Lambda)$. Also, conditions ensuring the existence of such splittings are found.

Let $\Lambda=\left\{\lambda_{k}, n_{k}\right\}_{k=1}^{\infty}$ be a multiple sequence of complex numbers with the only limit point $\infty$. The symbol $B(w, r)$ denotes the open disk centered at $w$ and of radius $r>0$, and $M_{\Lambda}(z, \delta)$ stands for the number of $\lambda_{k}$ 's (counted with multiplicity) that belong to $B(z, \delta|z|)$. For a cone $\Gamma$ with vertex at the origin, we put

$$
M_{\Lambda, \Gamma}(\delta)=\limsup _{z \in \Gamma,|z| \rightarrow \infty} \frac{M_{\Lambda}(z, \delta)}{|z|}, \quad M_{\Lambda, \Gamma}=\lim _{\delta \rightarrow 0} M_{\Lambda, \Gamma}(\delta) .
$$

The second identity is consistent because the function $M_{\Lambda, \Gamma}(\delta)$ is monotone. We omit the symbol $\Gamma$ in the above notation if $\Gamma=\mathbb{C}$. But if $\Gamma$ is the ray $\{t \xi, t>0\}$, $\xi \in \partial B(0,1)$, we replace $\Gamma$ with $\xi$ (for example, we write $M_{\Lambda, \xi}(\delta)$ in place of $M_{\Lambda, \Gamma}(\delta)$ ). Let $\lambda_{k} \in B(z, \delta|z|)$. Then $B(z, \delta|z|) \subset B\left(\lambda_{k}, 2(1-\delta)^{-1} \delta\left|\lambda_{k}\right|\right)$. Therefore,

$$
M_{\Lambda}(z, \delta) /|z| \leq(1+\delta) M_{\Lambda}\left(\lambda_{k}, 2(1-\delta)^{-1} \delta\right) /\left|\lambda_{k}\right|
$$

From this it easily follows that

$$
M_{\Lambda}=\lim _{\delta \rightarrow 0} \limsup _{k \rightarrow \infty} M_{\Lambda}\left(\lambda_{k}, \delta\right) /\left|\lambda_{k}\right| .
$$

Let $N(\Lambda)$ be the upper density of $\Lambda$, i.e.,

$$
N(\Lambda)=\limsup _{l \rightarrow \infty} l /\left|\xi_{l}\right|
$$

where $\left\{\xi_{l}\right\}$ is the sequence composed of the points $\lambda_{k}$ repeated $n_{k}$ times each. We denote by $N(\Lambda, r)$ the number of points $\lambda_{k}$ that belong to $B(0, r)$ (the multiplicities $n_{k}$ are taken into account). It can easily be verified that

$$
N(\Lambda)=\limsup _{r \rightarrow+\infty} N(\Lambda, r) / r
$$

Lemma 2.1. We have $M_{\Lambda}(\delta) \leq(1+\delta) N(\Lambda), \delta>0$. Moreover, if $M_{\Lambda}<\infty$, then $N(\Lambda)<\infty$. 
Proof. We have

$$
\begin{aligned}
M_{\Lambda}(\delta) & =\limsup _{|z| \rightarrow \infty} \frac{M_{\Lambda}(z, \delta)}{|z|} \leq \limsup _{|z| \rightarrow \infty} \frac{N(\Lambda,(1+\delta)|z|)}{|z|} \\
& =(1+\delta) \limsup _{r \rightarrow+\infty} \frac{N(\Lambda, r)}{r}=(1+\delta) N(\Lambda) .
\end{aligned}
$$

If $M_{\Lambda}<\infty$, then there exist numbers $\delta \in(0,1)$ and $R>0$ such that $M_{\Lambda}(z, 2 \delta) \leq$ $2 M_{\Lambda}|z|, z \in \mathbb{C} \backslash B(0, r)$. Let $|\xi|=1$, let $\Gamma(\xi, \widetilde{\delta})=\{t z: z \in B(\xi, \widetilde{\delta}), t>0\}$, and let $N(\Lambda, r, \xi, \widetilde{\delta})$ be the number of $\lambda_{k}$ 's (counted with multiplicities) in the sector $\Gamma(\xi, \widetilde{\delta}) \cap$ $\partial B(0,1) \subset B(0, r)$. We choose $\widetilde{\delta}>0$ in such a way that $\Gamma(\xi, \widetilde{\delta}) \cap \partial B(0,1) \subset B(\xi, \delta)$. Then the disk $B((1+\delta) t \xi, 2(1+\delta) \delta t)$ includes the set $\Gamma(\xi, \widetilde{\delta}) \cap(B(0,(1+\delta) t) \backslash B(0, t))$. Indeed, every $w$ in this set satisfies

$$
|w-(1+\delta) t \xi| \leq|w-| w|\xi|+\| w|\xi-(1+\delta) t \xi| \leq|w| \delta+\delta t<2(1+\delta) \delta t .
$$

Put $z_{m}=(1+\delta)^{m} R \xi, m \geq 1$, and denote by $m(r)$ the smallest number $m$ with $\left|z_{m}\right| \geq r$. We have

$$
\begin{aligned}
\limsup _{r \rightarrow+\infty} \frac{N(\Lambda, r, \xi, \tilde{\delta})}{r} & =\limsup _{r \rightarrow+\infty} \frac{N(\Lambda, r, \xi, \tilde{\delta})-N(\Lambda, R, \xi, \tilde{\delta})}{r} \\
& \leq \limsup _{r \rightarrow+\infty} \sum_{m=1}^{m(r)} \frac{M_{\Lambda}\left(z_{m}, 2 \delta\right)}{r} \leq 2 M_{\Lambda} \limsup _{r \rightarrow+\infty} \sum_{m=1}^{m(r)} \frac{\left|z_{m}\right|}{r} \\
& =2 M_{\Lambda} \limsup _{r \rightarrow+\infty} \sum_{m=1}^{m(r)} \frac{(1+\delta)^{m}}{(1+\delta)^{m(r)-1}} \leq 2 M_{\Lambda}\left(2+\delta+\frac{1+\delta}{\delta}\right) .
\end{aligned}
$$

This easily implies the claim.

We shall denote by $U=\left\{U_{m}\right\}_{m=1}^{\infty}$ a splitting of the sequence $\left\{\lambda_{k}\right\}$ into finite groups $U_{m}, m \geq 1$. We shall use the alternative notation $\lambda_{m, l}$ for the points $\lambda_{k}$ belonging to $U_{m}$, and the notation $n_{m, l}$ for their multiplicities. Here the first index coincides with the number of the group, and the second varies from 1 to $M_{m}$, where $M_{m}$ is the number of points $\lambda_{k}$ (without multiplicity) in $U_{m}$. Let $N_{m}$ be the number of $\lambda_{k}$ 's in $U_{m}$ with multiplicity taken into account, i.e., $N_{m}=\sum_{l=1}^{M_{m}} n_{m, l}$. We introduce the quantities

$$
\begin{aligned}
& \mathcal{N}(\Lambda, U)=\limsup _{m \rightarrow \infty} \max _{1 \leq p \leq M_{m}} N_{m} \backslash\left|\lambda_{m, p}\right|, \\
& \mathcal{D}(\Lambda, U)=\limsup _{m \rightarrow \infty} \max _{1 \leq j, l, p \leq M_{m}}\left|\lambda_{m, j}-\lambda_{m, l}\right| /\left|\lambda_{m, p}\right| .
\end{aligned}
$$

The groups $U_{m}$ are said to be relatively small if $\mathcal{D}(\Lambda, U)=\mathcal{N}(\Lambda, U)=0$.

Consider the function

$$
q_{\Lambda}(z, w, \delta)=\prod_{\lambda_{k} \in B(w, \delta|w|)}\left(\frac{z-\lambda_{k}}{3 \delta\left|\lambda_{k}\right|}\right)^{n_{k}} .
$$

If none of the $\lambda_{k}$ belongs to $B(w, \delta|w|)$, we put $q_{\Lambda}(z, w, \delta) \equiv 1$. The modulus of $q_{\Lambda}(z, w, \delta)$ can be interpreted as concentration near $z$ for the points $\lambda_{k} \in B(w, \delta|w|)$. Heuristically, the quantity $\ln \left|q_{\Lambda}(z, w, \delta)\right| /|w|$ is similar to the logarithm of the geometric mean (the arithmetic mean of the logarithms) of the normed distances from $\lambda_{k} \in B(w, \delta|w|)$ to $z$. If $\delta \in(0,1)$, then the modulus of each factor $q_{\Lambda}$ is estimated in $B(w, \delta|w|)$ by $2(3(1-\delta))^{-1}$ from above. Thus, it does not exceed 1 if $\delta \in(0,1 / 3)$. Next, if $\delta_{1} \leq \delta_{2}$ and $B\left(w_{1}, \delta_{1}\left|w_{1}\right|\right) \subseteq B\left(w_{2}, \delta_{2}\left|w_{2}\right|\right)$, then the factors $q_{\Lambda}\left(z, w_{1}, \delta_{1}\right)$ cannot 
outnumber the factors $q_{\Lambda}\left(z, w_{2}, \delta_{2}\right)$, and each factor $q_{\Lambda}\left(z, w_{1}, \delta_{1}\right)$ does not exceed the corresponding factor $q_{\Lambda}\left(z, w_{2}, \delta_{2}\right)$ in modulus. Consequently,

$$
\left|q_{\Lambda}\left(z, w_{1}, \delta_{1}\right)\right| \geq\left|q_{\Lambda}\left(z, w_{2}, \delta_{2}\right)\right|, \quad z \in B\left(w_{2}, \delta_{2}\left|w_{2}\right|\right),
$$

if $0<\delta_{1} \leq \delta_{2}<1 / 3$ and $B\left(w_{1}, \delta_{1}\left|w_{1}\right|\right) \subseteq B\left(w_{2}, \delta_{2}\left|w_{2}\right|\right)$. We put

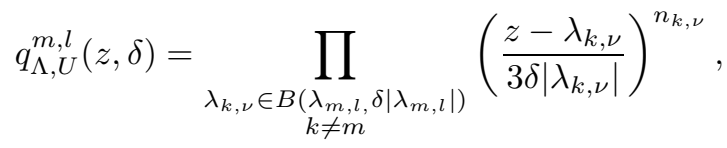

$$
\begin{aligned}
& m=1,2, \ldots, \quad l=1, \ldots, M_{m} .
\end{aligned}
$$

If the disk $B\left(\lambda_{m, l}, \delta\left|\lambda_{m, l}\right|\right)$ does not contain any of $\lambda_{k, \nu}, k \neq m$, we put $q_{\Lambda, U}^{m, l}(z, \delta) \equiv 1$. Let $m=1,2, \ldots, 1 \leq l$, and let $k \leq M_{m}$. As above, we obtain the inequality

$$
\left|q_{\Lambda, U}^{m, l}\left(z, \delta_{1}\right)\right| \geq\left|q_{\Lambda, U}^{m, k}\left(z, \delta_{2}\right)\right|, \quad z \in B\left(\lambda_{m, k}, \delta_{2}\left|\lambda_{m, k}\right|\right),
$$

if $0<\delta_{1} \leq \delta_{2}<1 / 3$ and $B\left(\lambda_{m, l}, \delta_{2}\left|\lambda_{m, l}\right|\right) \subseteq B\left(\lambda_{m, k}, \delta_{2}\left|\lambda_{m, k}\right|\right)$. We put

$$
\mathcal{S}_{\Lambda}(U)=\lim _{\delta \rightarrow 0} \liminf _{m \rightarrow \infty} \min _{1 \leq l \leq M_{m}} \frac{\ln \left|q_{\Lambda, U}^{m, l}\left(\lambda_{m, l}, \delta\right)\right|}{\left|\lambda_{m, l}\right|} .
$$

This definition is consistent because the limit in $\delta$ exists by (2.2). We observe that $\mathcal{S}_{\Lambda}(U) \leq 0$. Indeed, the quantity $\ln \left|q_{\Lambda, T}^{m, l}\left(\lambda_{m, l}, \delta\right)\right|$ is nonpositive for all $\delta \in(0,1 / 3)$. If the partition $U$ is trivial, i.e., each group $U_{m}$ is a singleton, then the quantity $\mathcal{S}_{\Lambda}(U)$ coincides with the quantity $\mathcal{S}_{\Lambda}$ introduced in [6]. If $M_{\Lambda}=0$, then $\mathcal{S}_{\Lambda}(U)$ does not change if the number 3 in the definition of $q_{\Lambda}$ is replaced with any other nonzero number (see the remark to Theorem 5.1 in [6]) in this connection). The coefficient 3 has been chosen for convenience only.

It can easily be observed that $\mathcal{N}(\Lambda, U) \leq M_{\Lambda}$ whenever $\mathcal{D}(\Lambda, U)=0$. But if $\mathcal{D}(\Lambda, U)<$ $\infty$, then $\mathcal{N}(\Lambda, U)$ is finite whenever $M_{\Lambda}$ is finite. Indeed, by Lemma 2.1, the condition $M_{\Lambda}<\infty$ implies $N(\Lambda)<\infty$. Then, invoking inequalities similar to those used at the beginning of the proof of that lemma, we deduce that $\mathcal{N}(\Lambda, U)<(1+\mathcal{D}(\Lambda, U)) N(\Lambda)$. The converse is not always true, i.e., $M_{\Lambda}$ may be infinite even if $\mathcal{N}(\Lambda, U)$ is finite. We introduce yet another quantity:

$$
\mathcal{N}_{1}(\Lambda, U)=\lim _{\delta \rightarrow 0} \limsup _{m \rightarrow \infty} \max _{1 \leq l \leq M_{m}} N_{m}\left(\lambda_{m, l}, \delta\right) /\left|\lambda_{m, l}\right|
$$

where $N_{m}(z, \delta)$ is the number (with multiplicity) of points of $U_{m}$ that lie in the disk $B(z, \delta|z|)$. Note that the quantities $\mathcal{N}(\Lambda, U)$ and $\mathcal{N}_{1}(\Lambda, U)$ coincide if $\mathcal{D}(\Lambda, U)=0$. We clearly have $\mathcal{N}_{1}(\Lambda, U) \leq M_{\Lambda}$. It turns out that the converse is also true if the groups are separated. More precisely, we have the following statement.

Lemma 2.2. Let the sequence $\Lambda=\left\{\lambda_{k}, n_{k}\right\}_{k=1}^{\infty}$ be split into groups $U=\left\{U_{m}\right\}_{m=1}^{\infty}$, where $U_{m}=\left\{\lambda_{m, l}\right\}_{l=1}^{M_{m}}$. Suppose that $\mathcal{S}_{\Lambda}(U)>-\infty$. Then

$$
\liminf _{\delta \rightarrow 0} \ln \delta \limsup _{m \rightarrow \infty} \max _{1 \leq l \leq M_{m}} \frac{M_{\Lambda}\left(\lambda_{m, l}, \delta\right)-N_{m}\left(\lambda_{m, l}, \delta\right)}{\left|\lambda_{m, l}\right|} \geq \mathcal{S}_{\Lambda}(U) .
$$

Proof. Fix $\varepsilon>0$. By the definition of $\mathcal{S}_{\Lambda}(U)$, we can choose $\alpha \in(0,1 / 3)$ and an index $m_{0}$ such that

$$
\min _{1 \leq l \leq M_{m}} \ln \left|q_{\Lambda, U}^{m, l}\left(\lambda_{m, l}, \alpha\right)\right| /\left|\lambda_{m, l}\right| \geq \mathcal{S}_{\Lambda}(U)-\varepsilon, \quad m \geq m_{0}
$$


Let $\delta \in(0, \alpha)$, and let $m \geq m_{0}$. If $M_{\Lambda}\left(\lambda_{m, l}, \delta\right)-N_{m}\left(\lambda_{m, l}, \delta\right)>0$, then, much as in the proof of inequality (2.1), we obtain

$$
\frac{1}{\left|\lambda_{m, l}\right|} \sum_{\substack{\lambda_{k, \nu} \in B\left(\lambda_{m, l} \delta\left|\lambda_{m, l}\right|\right), k \neq m}} n_{k, \nu} \ln \frac{\left|\lambda_{m, l}-\lambda_{k, \nu}\right|}{3 \alpha\left|\lambda_{k, \nu}\right|} \geq \frac{\ln \left|q_{\Lambda, U}^{m, l}\left(\lambda_{m, l}, \alpha\right)\right|}{\left|\lambda_{m, l}\right|} \geq \mathcal{S}_{\Lambda}(U)-\varepsilon .
$$

Consequently,

$$
\begin{aligned}
\mathcal{S}_{\Lambda}(U)-\varepsilon & \leq \frac{1}{\left|\lambda_{m, l}\right|} \sum_{\substack{\lambda_{k, \nu} \in B\left(\lambda_{m, l}, \delta\left|\lambda_{m, l}\right|\right) \\
k \neq m}} n_{k \nu} \ln \frac{\delta\left|\lambda_{m, l}\right|}{3 \alpha\left|\lambda_{k, \nu}\right|} \\
& \leq \frac{M_{\Lambda}\left(\lambda_{m, l}, \delta\right)-N_{m}\left(\lambda_{m, l}, \delta\right)}{\left|\lambda_{m, l}\right|} \ln \frac{\delta}{3 \alpha(1-\delta)}
\end{aligned}
$$

Thus,

$$
\ln \frac{\delta}{3 \alpha(1-\delta)} \limsup _{m \rightarrow \infty} \max _{1 \leq l \leq M_{m}} \frac{M_{\Lambda}\left(\lambda_{m, l}, \delta\right)-N_{m}\left(\lambda_{m, l}, \delta\right)}{\left|\lambda_{m, l}\right|} \geq \mathcal{S}_{\Lambda}(U)-\varepsilon, \quad \delta \in(0, \alpha) .
$$

Since $\varepsilon>0$ is arbitrary, the claim follows.

The inequality $\mathcal{S}_{\Lambda}(U)>-\infty$ means that the groups $U_{m}$ are mutually separated in a sense. The next statement clarifies the nature of this separation.

Lemma 2.3. Let $\Lambda$ be split into groups $U=\left\{U_{m}\right\}$, where $U_{m}=\left\{\lambda_{m, l}\right\}_{l=1}^{M_{m}}$. Suppose that $\mathcal{S}_{\Lambda}(U)>-\infty, \mathcal{N}(\Lambda, U)$ and $\mathcal{D}(\Lambda, U)$ are finite. Then there exists a sequence $\left\{\gamma_{m, l}\right\}_{l=1, m=1}^{M_{m}, \infty}$ of positive numbers such that

1)

$$
\begin{gathered}
\liminf _{m \rightarrow \infty} \min _{1 \leq l, j \leq M_{m}} \ln \gamma_{m, l} /\left|\lambda_{m, j}\right| \geq(1+\mathcal{D}(\Lambda, U)) \mathcal{S}_{\Lambda}(U) ; \\
\limsup _{m \rightarrow \infty} \max _{1 \leq l, j \leq M_{m}} \lambda_{m, l} /\left|\gamma_{m, j}\right| \leq \mathcal{N}(\Lambda, U)+\mathcal{D}(\Lambda, U)
\end{gathered}
$$

2) the sets $B_{m}=\bigcup_{l=1}^{M_{m}} B\left(\lambda_{m, l}, \gamma_{m, l}\right), m \geq 1$, are mutually disjoint;

3) the diameters $d_{m}$ of the sets $B_{m}$ satisfy the relation

$$
\limsup _{m \rightarrow \infty} \max _{1 \leq p \leq M_{m}} d_{m} /\left|\lambda_{m, p}\right| \leq 2(\mathcal{N}(\Lambda, U)+\mathcal{D}(\Lambda, U))
$$

4) for every $\varepsilon>0$ and $\theta \in(0,1)$ there exist quantities $R>0$ and $\delta \in(0,1 / 3)$ such that $\ln \left|q_{\Lambda}(z, w, \delta)\right| \geq\left(2 \mathcal{S}_{\Lambda}(U)-\varepsilon\right)|z|$ for $z \in\left(B_{m} \backslash \widetilde{B}_{m}(\theta)\right) \cap B(w, \delta|w|),|w| \geq R$, and $m \geq 1$, where $\widetilde{B}_{m}(\theta)=\bigcup_{l=1}^{M_{m}} B\left(\lambda_{m, l}, \theta \gamma_{m, l}\right)$.

Proof. We define $\gamma_{m, l}$. For every $m \geq 1, l=1, \ldots, M_{m}$, we denote by $\beta_{m, l}$ the minimal distance from $\lambda_{m, l}$ to the points $\lambda_{s, j}$ not belonging to $U_{m}$. Fix $m \geq 1$. If $N_{m} \leq \beta_{m, l} / 2$ for all $l=1, \ldots, M_{m}$, we put $\gamma_{m, l}=N_{m}, l=1, \ldots, M_{m}$. Otherwise, we put $\gamma_{m, l}=\beta_{m, l} / 2$, $l=1, \ldots, M_{m}$. We show that statements 1)-4) are fulfilled. By construction, the disks $B\left(\lambda_{m, l}, \gamma_{m, l}\right)$ and $B\left(\lambda_{s, j}, \gamma_{s, j}\right)$ do not intersect if $m \neq s$. Consequently, the sets $B_{m}$, $m \geq 1$, are mutually disjoint. Thus proves 2$)$. We verify 3$)$. Observe that the distance between arbitrary points $z_{1}$ and $z_{2}$ in $B_{m}$ is at most $\left|\lambda_{m, l}-\lambda_{m, j}\right|+\gamma_{m, l}+\gamma_{m, j}$, where $l$ and $j$ are such that $z_{1}$ and $z_{2}$ belong to $B\left(\lambda_{m, l}, \gamma_{m, l}\right)$ and $B\left(\lambda_{m, j}, \gamma_{m, j}\right)$, respectively. Then

$$
d_{m} \leq \max _{1 \leq l, s \leq M_{m}}\left|\lambda_{m, l}-\lambda_{m, s}\right|+2 \max _{1 \leq l \leq M_{m}} \gamma_{m, l} .
$$

The definition of $\gamma_{m, l}$ shows that either $\gamma_{m, l}=N_{m}$ or $\gamma_{m, l}=\beta_{m, l} / 2$ for all $l=1, \ldots, M_{m}$. In the first case we have

$$
\max _{1 \leq l \leq M_{m}} \gamma_{m, l}=N_{m}
$$


In the second case there is at least one index $j=1, \ldots, M_{m}$ with $N_{m}>\beta_{m, j} / 2$. Then the definition of $\beta_{m, l}$ and the triangle inequality show that

$$
\gamma_{m, l}=\beta_{m, l} / 2 \leq \beta_{m, j} / 2+\left|\lambda_{m, l}-\lambda_{m, j}\right| / 2 \leq N_{m}+\left|\lambda_{m, l}-\lambda_{m, j}\right| / 2 .
$$

Thus, in either case we have

$$
d_{m} \leq 2 \max _{1 \leq l, j \leq M_{m}}\left|\lambda_{m, l}-\lambda_{m, j}\right|+2 N_{m}, \quad m=1,2, \ldots
$$

This yields 3). We prove 1). By (2.3), (2.4), and the definitions of $\mathcal{N}(\Lambda, U)$ and $\mathcal{D}(\Lambda, U)$, we obtain

$$
\limsup _{m \rightarrow \infty} \max _{1 \leq l, j \leq M_{m}} \gamma_{m, l} /\left|\lambda_{m, j}\right| \leq \mathcal{N}(\Lambda, U)+\mathcal{D}(\Lambda, U) .
$$

On the other hand, if $\gamma_{m(p), l}=N_{m(p)}, p \geq 1$, then

$$
\liminf _{p \rightarrow \infty} \min _{1 \leq l, j \leq M_{m(p)}} \ln \gamma_{m(p), j} /\left|\lambda_{m(p), j}\right|=0 .
$$

Let $\gamma_{m(p), l}=\beta_{m(p), l} / 2, l=1, \ldots, M_{m(p)}$. Using the definition of $\beta_{m, l}$ and the fact that, for $\delta \in(0,1 / 3)$, the modulus of every factor involved in $\left.q_{\Lambda, U}^{m, l}(\lambda, \delta)\right)$ does not exceed 1 in the disk $B\left(\lambda_{m, l}, \delta\left|\lambda_{m, l}\right|\right)$, we see that

$$
\begin{aligned}
\liminf _{p \rightarrow \infty} \min _{1 \leq l, j \leq M_{m(p)}} \frac{\ln \gamma_{m(p), l}}{\left|\lambda_{m(p), j}\right|} & =\liminf _{p \rightarrow \infty} \min _{1 \leq l, j \leq M_{m}} \frac{\ln \beta_{m(p), l}}{\left|\lambda_{m(p), j}\right|} \\
& \geq \liminf _{p \rightarrow \infty} \min _{1 \leq l, j \leq M_{m(p)}}\left(\frac{\ln \left|q_{\Lambda, U}^{m(p), l}\left(\lambda_{m, l}, \delta\right)\right|}{\left|\lambda_{m(p), l}\right|}\right) \frac{\left|\lambda_{m(p), l}\right|}{\left|\lambda_{m(p), j}\right|} \\
& \geq\left(1+\mathcal{D}(\Lambda, U) \liminf _{m \rightarrow \infty} \min _{1 \leq l \leq M_{m}} \frac{\ln \left|q_{\Lambda, U}^{m, l}\left(\lambda_{m, l}, \delta\right)\right|}{\left|\lambda_{m, l}\right|}\right.
\end{aligned}
$$

for such $\delta$. By the definition of $\mathcal{S}_{\Lambda}(U)$, property 1) follows.

It remains to establish property 4). Suppose, to the contrary, that 4) is violated. Then for some $\varepsilon>0$ and $\theta \in(0,1)$ we can find numerical sequences $\left\{w_{k}\right\},\left\{z_{k}\right\}$ and a sequence of sets $B_{m(k)} \backslash \widetilde{B}_{m(k)}(\theta)$ in such a way that $m(k) \rightarrow \infty, z_{k} \in\left(B_{m(k)} \backslash \widetilde{B}_{m(k)}(\theta)\right) \cap$ $B\left(w_{k},\left|w_{k}\right| / k\right)$, and

$$
\ln \left|q_{\Lambda}\left(z_{k}, w_{k}, k^{-1}\right)\right| \leq\left(2 \mathcal{S}_{\Lambda}(U)-\varepsilon\right)\left|z_{k}\right|, \quad k=1,2, \ldots
$$

Combined with the definition of $q_{\Lambda}$, this shows that, for every $k \geq 1$, the set $B\left(w_{k},\left|w_{k}\right| / k\right)$ contains at least one point $\lambda_{m, l}$. We prove that (2.5) contradicts the assumptions of the lemma. For this, we must estimate two groups of factors that form the quantity $\left|q_{\Lambda}\left(z_{k}, w_{k}, k^{-1}\right)\right|$ from below. The first group corresponds to the points $\lambda_{m, l} \in$ $B\left(w_{k}\left|w_{k}\right| / k\right)$ with $m \neq m(k)$, and the second corresponds to the points $\lambda_{m(k), l} \in$ $B\left(w_{k},\left|w_{k}\right| / k\right)$. We estimate the first group. By assumption, $\mathcal{S}_{\Lambda}(U)>-\infty$. Then, by the definition of $\mathcal{S}_{\Lambda}(U)$, there exists $\delta \in(0,1 / 3)$ and an index $m_{0}$ such that

$$
\min _{1 \leq l \leq M_{m}} \ln \left|q_{\Lambda, U}^{m, l}\left(\lambda_{m, l}, \delta\right)\right| /\left|\lambda_{m, l}\right| \geq \mathcal{S}_{\Lambda}(U)-\varepsilon / 4, \quad m \geq m_{0}
$$

Since $z_{k} \in B_{m(k)}$, the definition of $B_{m}$ shows that for every $k \geq 1$ there exists an index $l(k)$ with $z_{k} \in B\left(\lambda_{m(k), l(k), l(k)}, \gamma_{m(k), l(k)}\right)$. We fix $k \geq 1$. Let $\lambda_{m, l} \in B\left(w_{k},\left|w_{k}\right| / k\right)$ fall out from $U_{m(k)}$. Then from the definitions of $\beta_{m(k), l(k)}$ and $\gamma_{m(k), l(k)}$ it follows that

$$
\begin{aligned}
\beta_{m(k), l(k)} & \leq\left|\lambda_{m(k), l(k)}-\lambda_{m, l}\right| \leq\left|\lambda_{m(k), l(k)}-z_{k}\right|+\left|z_{k}-\lambda_{m, l}\right| \\
& \leq \gamma_{m(k), l(k)}+2\left|w_{k}\right| / k \leq \beta_{m(k), l(k)} / 2+2\left|w_{k}\right| / k .
\end{aligned}
$$

This yields the estimate $\gamma_{m(k), l(k)} \leq \beta_{m(k), l(k)} / 2 \leq 2\left|w_{k}\right| / k$. Furthermore, we have

$$
\left|w_{k}\right| \leq\left|w_{k}-z_{k}\right|+\left|z_{k}-\lambda_{m(k), l(k)}\right|+\left|\lambda_{m(k), l(k)}\right| \leq\left|w_{k}\right| / k+\gamma_{m(k), l(k)}+\left|\lambda_{m(k), l(k)}\right| .
$$


Consequently, $\left|\lambda_{m(k), l(k)}-\lambda_{m, l}\right| \leq 4\left|w_{k}\right| / k$ and $\left|w_{k}\right| \leq 3\left|w_{k}\right| / k+\left|\lambda_{m(k), l(k)}\right|$. This means that the point $\lambda_{m, l}$ belongs to $B\left(\lambda_{m(k), l(k)}, a_{k}\left|\lambda_{m(k), l(k)}\right|\right)$, where $a_{k}=4 /(k-3)$.

We choose $k_{0}$ so as to have $a_{k}<\delta$ and $m(k) \geq m_{0}$ for all $k \geq k_{0}$. Fixing $k \geq k_{0}$, take $\lambda_{m, l} \in B\left(w_{k},\left|w_{k}\right| / k\right), m \neq m(k)$. Then $\lambda_{m, l} \in B\left(\lambda_{m(k), l(k)}, \delta\left|\lambda_{m(k), l(k)}\right|\right)$. Therefore, using (2.6), the definition of $q_{\Lambda, U}^{m(k), l(k)}$, the properties of the factors involved in the last quantity, and the fact that $\delta \in(0,1 / 3)$, we obtain

$$
\begin{aligned}
\prod_{\substack{\lambda_{m, l} \in B\left(w_{k},\left|w_{k}\right| / k\right) \\
m \neq m(k)}}\left(\frac{\left|\lambda_{m(k), l(k)}-\lambda_{m, l}\right|}{3 \delta\left|\lambda_{m, l}\right|}\right)^{n_{m, l}} & \geq\left|q_{\Lambda, U}^{m(k), l(k)}\left(\lambda_{m(k), l(k)}, \delta\right)\right| \\
& \geq \exp \left(\left|\lambda_{m(k), l(k)}\right|\left(\mathcal{S}_{\Lambda}(U)-\varepsilon / 4\right)\right) .
\end{aligned}
$$

The preceding estimates easily show that $\left|\lambda_{m(k), l(k)}\right| /\left|z_{k}\right| \rightarrow 1$ as $k \rightarrow \infty$. Thus, for some $k_{1} \geq k_{0}$ we have

$$
\prod_{\substack{\lambda_{m, l} \in B\left(w_{k},\left|w_{k}\right| / k\right) \\ m \neq m(k)}}\left(\frac{\left|\lambda_{m(k), l(k)}-\lambda_{m, l}\right|}{3 \delta\left|\lambda_{m, l}\right|}\right)^{n_{m, l}} \geq \exp \left(\left|z_{k}\right|\left(\mathcal{S}_{\Lambda}(U)-\varepsilon / 4\right)\right), \quad k \geq k_{1} .
$$

Let $m \neq m(k)$. Since $z_{k} \in B\left(\lambda_{m(k), l(k)}, \gamma_{m(k), l(k)}\right)$, from the definition of $\gamma_{m(k), l(k)}$ and $\beta_{m(k), l(k)}$ we deduce that

$$
\left|z_{k}-\lambda_{m, l}\right| \geq\left|\lambda_{m(k), l(k)}-\lambda_{m, l}\right|-\beta_{m(k), l(k)} / 2 \geq\left|\lambda_{m(k), l(k)}-\lambda_{m, l}\right| / 2 .
$$

Together with (2.7), this shows that if $k_{2} \geq \max \left\{k_{1}, 2 \delta^{-1}\right\}$, then

$$
\prod_{\substack{\lambda_{m, l} \in B\left(w_{k},\left|w_{k}\right| / k\right) \\ m \neq m(k)}}\left(\frac{\left|z_{k}-\lambda_{m, l}\right|}{3 k^{-1}\left|\lambda_{m, l}\right|}\right)^{n_{m, l}} \geq \exp \left(\left|z_{k}\right|\left(\mathcal{S}_{\Lambda}(U)-\varepsilon / 4\right)\right), \quad k \geq k_{2} .
$$

Now we estimate the second group of factors involved in $\left|q_{\Lambda}\left(z_{k}, w_{k}, k^{-1}\right)\right|$, namely, the factors corresponding to the points $\lambda_{m(k), l} \in B\left(w_{k},\left|w_{k}\right| / k\right)$. Passing to a subsequence, we may assume that one of the following two cases occurs:

1) $\gamma_{m(k), l}=N_{m(k)}, l=1, \ldots, M_{m(k)}, k \geq 1$;

2) $\gamma_{m(k), l}=\beta_{m(k), l} / 2, l=1, \ldots, M_{m(k)}, k \geq 1$.

Consider the first case. Since $z_{k} \notin \widetilde{B}_{m(k)}(\theta)$ and $z_{k} \in B\left(w_{k},\left|w_{k}\right| / k\right)$, we obtain

$$
\begin{gathered}
\prod_{\lambda_{m(k), l} \in B\left(w_{k},\left|w_{k}\right| / k\right)}\left|\left(\frac{z_{k}-\lambda_{m(k), l}}{3 k^{-1}\left|\lambda_{m(k), l}\right|}\right)\right|^{n_{m(k), l}} \geq \\
\geq \prod_{\lambda_{m(k), l} \in B\left(w_{k},\left|w_{k}\right| / k\right)}\left(\frac{\theta N_{m(k)}}{3 k^{-1}\left|\lambda_{m(k), l}\right|}\right)^{n_{m(k), l}} \\
\prod_{\lambda_{m(k), l} \in B\left(w_{k},\left|w_{k}\right| / k\right)}\left(\frac{\left(1-k^{-1}\right) \theta N_{m(k)}}{3 k^{-1}\left(1+k^{-1}\left|z_{k}\right|\right)}\right)^{n_{m(k), l}}, \quad k \geq 1 .
\end{gathered}
$$

It follows that

$$
\frac{1}{\left|z_{k}\right|} \sum_{\lambda_{m(k), l} \in B\left(w_{k},\left|w_{k}\right| / k\right)} \ln \left|\left(\frac{z_{k}-\lambda_{m(k), l}}{3 k^{-1}\left|\lambda_{m(k), l}\right|}\right)\right|^{n_{m(k), l}} \geq \inf _{x>0} x \ln \left(\frac{k \theta\left(1-k^{-1}\right) x}{3\left(1+k^{-1}\right)}\right) \rightarrow 0, \quad k \rightarrow \infty
$$

Thus, there exists $k_{3} \geq k_{2}$ such that

$$
\prod_{\lambda_{m(k), l} \in B\left(w_{k},\left|w_{k}\right| / k\right)}\left|\left(\frac{z_{k}-\lambda_{m(k), l}}{3 k^{-1}\left|\lambda_{m(k), l}\right|}\right)\right|^{n_{m(k), l}} \geq \exp \left(-\left|z_{k}\right| \varepsilon / 4\right), \quad k \geq k_{3} .
$$

Finally, we consider the case where $\gamma_{m(k), l}=\beta_{m(k), l} / 2$ for all $k$ and $l$. Fix $k \geq 1$. By using the definition of $\beta_{m(k), l(k)}$, we find indices $p(k) \neq m(k)$ and $j(k)$ such that 
$\left|\lambda_{m(k), l(k)}-\lambda_{p(k), j(k)}\right|=\beta_{m(k), l(k)}$. For every $l=1, \ldots, M_{m(k)}$, we have

$$
\begin{aligned}
\left|\lambda_{m(k), l}-\lambda_{p(k), j(k)}\right| & \leq\left|\lambda_{m(k), l}-z_{k}\right|+\left|z_{k}-\lambda_{m(k), l(k)}\right|+\left|\lambda_{m(k), l(k)}-\lambda_{p(k), j(k)}\right| \\
& \leq\left|\lambda_{m(k), l}-z_{k}\right|+\beta_{m(k), l(k)} / 2+\beta_{m(k), l(k)} \\
& =\left|\lambda_{m(k), l}-z_{k}\right|+3 \beta_{m(k), l(k)} / 2 .
\end{aligned}
$$

Take $\lambda_{m(k), l} \in B\left(w_{k},\left|w_{k}\right| / k\right)$ and $s(k) \neq m(k)$ and $i$ such that $\left|\lambda_{m(k), l}-\lambda_{s(k), i}\right|=\beta_{m(k), l}$. Then

$$
\begin{aligned}
\beta_{m(k), l(k)} \leq\left|\lambda_{m(k), l(k)}-\lambda_{s(k), i}\right| & \leq\left|z_{k}-\lambda_{m(k), l(k)}\right|+\left|\lambda_{m(k), l}-z_{k}\right|+\left|\lambda_{m(k), l}-\lambda_{s(k), i}\right| \\
& \leq \beta_{m(k), l(k)} / 2+\left|\lambda_{m(k), l}-z_{k}\right|+\beta_{m(k), l} .
\end{aligned}
$$

Since $z_{k} \notin \widetilde{B}_{m(k)}(\theta)$, we have $\left|\lambda_{m(k), l}-z_{k}\right| \geq \theta \beta_{m(k), l} / 2$. Suppose that $\left|\lambda_{m(k), l}-z_{k}\right| \leq$ $\theta \beta_{m(k), l(k)} / 6$. Then $\beta_{m(k), l} \leq \beta_{m(k), l(k)} / 3$. By the above, this implies the estimate $\beta_{m(k), l(k)} \leq \beta_{m(k), l(k)} / 2+\theta \beta_{m(k), l(k)} / 6+\beta_{m(k), l(k)} / 3$, a contradiction. Thus, $\mid \lambda_{m(k), l}-$ $z_{k} \mid>\theta \beta_{m(k), l(k)} / 6$, and by (2.10) we obtain

$$
\frac{\left|\lambda_{m(k), l}-\lambda_{p(k), j(k)}\right|}{\left|\lambda_{m(k), l}-z_{k}\right|} \leq 1+\frac{3 \beta_{m(k), l(k)}}{2\left|\lambda_{m(k), l}-z_{k}\right|}<\frac{9}{\theta} .
$$

Consequently, $\left|\lambda_{m(k), l}-\lambda_{p(k), j(k)}\right|<9\left|\lambda_{m(k), l}-z_{k}\right| / \vartheta<18\left|w_{k}\right| / \theta k$. Moreover,

$$
\left|w_{k}-\lambda_{p(k), j(k)}\right| \leq\left|w_{k}-\lambda_{m(k), l}\right|+\left|\lambda_{m(k), l}-\lambda_{p(k), j(k)}\right|<2\left|w_{k}\right| / k+18\left|w_{k}\right| / \theta k .
$$

This means that, for some $k_{4} \geq k_{3}$ and all $k \geq k_{4}$, the points $\lambda_{m(k), l}$ belonging to $B\left(w_{k},\left|w_{k}\right| / k\right)$ belong also to $B\left(\lambda_{p(k), j(k)}, \delta\left|\lambda_{p(k), j(k)}\right|\right)$. We may assume that $9(\theta k)^{-1}<\delta$. Therefore, by (2.11) and the properties of the factors that form $q_{\Lambda, U}^{p(k), j(k)}(z, \delta)$, we obtain

$$
\begin{aligned}
\prod_{\lambda_{m(k), l} \in B\left(w_{k},\left|w_{k}\right| / k\right)}\left|\left(\frac{z_{k}-\lambda_{m(k), l}}{3 k^{-1}\left|\lambda_{m(k), l}\right|}\right)\right|^{n_{m(k), l}} \\
\quad \geq \prod_{\lambda_{m(k), l} \in B\left(w_{k},\left|w_{k}\right| / k\right)} \mid\left(\frac{\lambda_{p(k), j(k)}-\lambda_{m(k), l}}{27(\theta k)^{-1}\left|\lambda_{m(k), l}\right|}\right)^{n_{m(k), l}} \\
\geq \prod_{\lambda_{m(k), l} \in B\left(w_{k},\left|w_{k}\right| / k\right)}\left|\left(\frac{\lambda_{p(k), j(k)}-\lambda_{m(k), l}}{3 \delta\left|\lambda_{m(k), l}\right|}\right)\right|^{n_{m(k), l}} \\
\geq\left|q_{\Lambda, U}^{p(k), j(k)}\left(\lambda_{p(k), j(k), \delta}\right)\right|, \quad k \geq k_{4} .
\end{aligned}
$$

Since $\left|z_{k}\right| /\left|\lambda_{p(k), j(k)}\right| \rightarrow 1$ as $k \rightarrow \infty$, by (2.6) there exists $k_{3} \geq k_{4}$ such that

$$
\prod_{\lambda_{m(k), l} \in B\left(w_{k},\left|w_{k}\right| / k\right)}\left|\left(\frac{z_{k}-\lambda_{m(k), l}}{3 k^{-1}\left|\lambda_{m(k), l}\right|}\right)\right|^{n_{m(k), l}} \geq \exp \left(\left|z_{k}\right|\left(\mathcal{S}_{\Lambda}(U)-\varepsilon / 2\right)\right), \quad k \geq k_{5} .
$$

Taking (2.8) and (2.9) into account, we obtain

$$
\ln \left|q_{\Lambda}\left(z_{k}, w_{k}, k^{-1}\right)\right| \geq\left|z_{k}\right|\left(2 \mathcal{S}_{\Lambda}(U)-3 \varepsilon / 4\right), \quad k \geq k_{5} .
$$

This contradicts (2.5). Consequently, we have proved property 4) and, with it, the lemma.

A converse to Lemma 2.3 also holds, in the following form.

Lemma 2.4. Let the sequence $\Lambda=\left\{\lambda_{k}, n_{k}\right\}_{k=1}^{\infty}$ be such that there exists a sequence of open sets $B_{m}, m \geq 1$, with the following properties.

1) The sets $B_{m}, m=1,2, \ldots$, are mutually disjoint, their union contains all $\lambda_{k}$, and each $B_{m}$ contains at least one of the $\lambda_{k}$. 
2) The diameters $d_{m}$ of the sets $B_{m}, m=1,2, \ldots$, satisfy the condition

$$
\limsup _{m \rightarrow \infty} \sup _{\lambda \in B_{m}} d_{m} /|\lambda|=d<\infty \text {. }
$$

3) There is $a>0$ such that for every $\varepsilon>0$ there exist numbers $R>0$ and $\delta \in$ $(0,1 / 3)$ for which $\ln \left|q_{\Lambda}(z, w, \delta)\right| \geq-(a+\varepsilon)|z|, z \in \partial B_{m} \cap B(w, \delta|w|), m=1,2, \ldots$, $w \in \mathbb{C} \backslash B(0, R)$.

Define a splitting $U=\left\{U_{m}\right\}_{m=1}^{\infty}$ of $\Lambda$, where $U_{m}=\left\{\lambda_{m, l}\right\}_{l=1}^{M_{m}}$ consists of all points $\lambda_{k}$ belonging to $B_{m}$. Then $\mathcal{S}_{\Lambda}(U)>-a, \mathcal{D}(\Lambda, U) \leq d$.

Proof. The inequality $\mathcal{D}(\Lambda, U) \leq d$ is a direct consequence of the definitions of $d$ and $\mathcal{D}(\Lambda, U)$. We prove that $\mathcal{S}_{\Lambda}(U) \geq-a$. Fixing $\varepsilon>0$, we find $R>0$ and $\delta \in(0,1 / 3)$ as in item 3) of the lemma. By the definition of the quantities $q_{\Lambda, U}^{m, l}$ and $q_{\Lambda}$ and the properties of the factors they involve, it follows that

$$
\ln \left|q_{\Lambda, U}^{m, l}(z, \tilde{\delta})\right| \geq \ln \left|q_{\Lambda, U}^{m, l}(z, 5 \tilde{\delta})\right| \geq \ln \left|q_{\Lambda}\left(z, \lambda_{m, l}, 5 \tilde{\delta}\right)\right| \geq \ln \left|q_{\Lambda}\left(z, \lambda_{m, l}, \delta\right)\right| \geq-(a+\varepsilon)|z|,
$$

where $z \in \partial B_{m} \cap B\left(\lambda_{m, l}, \delta\left|\lambda_{m, l}\right|\right)$. Moreover, the definition of $q_{\Lambda, U}^{m, l}$ shows that

$$
\ln \left|q_{\Lambda, U}^{m, l}(z, \tilde{\delta})\right| \geq 0, \quad z \in \partial B\left(\lambda_{m, l}, 5 \tilde{\delta}\left|\lambda_{m, l}\right|\right) .
$$

Let $B_{m, l}$ be the component of $B_{m} \cap B\left(\lambda_{m, l}, 5 \widetilde{\delta}\left|\lambda_{m, l}\right|\right)$ that contains $\lambda_{m, l}$. Then the last relations imply

$$
\ln \left|q_{\Lambda, U}^{m, l}(z, \widetilde{\delta})\right| \geq-(a+\varepsilon)|z| \geq-(a+\varepsilon)(1+5 \widetilde{\delta})\left|\lambda_{m, l}\right|, \quad z \in \partial B_{m, l}
$$

Since $q_{\Lambda, U}^{m, l}(z, \tilde{\delta})$ does not vanish on $B_{m, l}$, the above inequality extends to this set. In particular, $\ln \left|q_{\Lambda, U}^{m, l}\left(\lambda_{m, l}, \tilde{\delta}\right)\right| \geq-(a+\varepsilon)(1+5 \tilde{\delta})\left|\lambda_{m, l}\right|$. It follows that

$$
\mathcal{S}_{\Lambda}(U)=\lim _{\delta \rightarrow 0} \liminf _{m \rightarrow \infty} \min _{1 \leq l \leq M_{m}} \ln \left|q_{\Lambda, U}^{m, l}\left(\lambda_{m, l}, \tilde{\delta}\right)\right| /\left|\lambda_{m, l}\right| \geq-a-\varepsilon .
$$

Since $\varepsilon>0$ is arbitrary, we see that $\mathcal{S}_{\Lambda}(U) \geq-a$.

Now, we indicate certain conditions on $\Lambda$ that ensure the existence of a splitting $U$ with $\mathcal{S}_{\Lambda}(U)>-\infty$. We start with an auxiliary statement.

Lemma 2.5. Suppose that $\Lambda=\left\{\lambda_{k}, n_{k}\right\}_{k=1}^{\infty}$ has finite upper density $N(\Lambda)$. Then for every $d>0$ there exist two numbers $a>0$ and $\delta \in(0,1 / 3)$, a sequence of domains $B_{m}$, $m \geq 1$, and numbers $R_{p}>0, p \geq 1$, with the following properties.

1) The sets $B_{m}, m=1,2, \ldots$, are mutually disjoint, their union contains all $\lambda_{k}$, and each $B_{m}$ contains at least one $\lambda_{k}$.

2) The diameters $d_{m}$ of $B_{m}, m=1,2, \ldots$, satisfy the condition

$$
\limsup _{m \rightarrow \infty} \sup _{z \in B_{m}} d_{m} /|z| \leq d,
$$

and we have

$$
R_{p+1} \leq(1+d) R_{p}, \quad R_{p+2} \geq(1+d / 5) R_{p}, \quad p=1,2, \ldots
$$

3) $\ln \left|q_{\Lambda}(z, w, \delta)\right| \geq-a|z|$ whenever $z \in\left(\partial B_{m} \cup \partial B\left(0, R_{p}\right)\right) \cap B(w, \delta|w|), m, p=$ $1,2, \ldots,|w| \geq 2$.

Proof. We fix a positive number $\alpha<\min \{d / 5,1 / 30\}$. Since $N(\Lambda)<\infty$, there exists $c>0$ such that

$$
M_{\Lambda}(z, 8 \alpha) \leq c|z|, \quad|z| \geq 1
$$

We choose $\varphi \in(0, \pi / 2)$ such that the set

$$
\left\{z=r e^{i \tau}: \tau \in[-\varphi / 2, \varphi / 2], r \geq 1\right\}
$$


is included in the union $\bigcup_{p \geq 1} B\left((1+\alpha)^{p-1}, \alpha(1+\alpha)^{p-1}\right)$. Denote by $l_{0}$ the greatest positive integer $l$ with $l \varphi<2 \pi$. Put $z_{p, l}=(1+\alpha)^{p-1} e^{i l \varphi}, p \geq 1, l=0, \ldots, l_{0}$. Then the union of $B(0,1)$ and all disks $B\left(z_{p, l}, \alpha\left|z_{p, l}\right|\right)$ covers the plane.

Let $\tilde{a}=c\left(1+\ln \left(256\left(l_{0}+1\right)\right)\right)$. For every $p \geq 1$ and $l=0, \ldots, l_{0}$, put

$$
\widetilde{B}^{p, l}=\left\{z \in \mathbb{C}: \ln \left|q_{\Lambda}\left(z, z_{p, l}, 3 \alpha\right)\right|<-2 \widetilde{a}\left|z_{p, l}\right|\right\} .
$$

We define $B^{p, l}$ as the union of all connected components of $\widetilde{B}^{p, l}$ that intersect the disk $B\left(z_{p, l}, \alpha\left|z_{p, l}\right|\right)$ and contain at least one point $\lambda_{k}$. Set

$$
B=\bigcup_{p=1, l=0}^{\infty, l_{0}} B^{p, l} \cup B(0,1)
$$

If the disk $B(0,1)$ contains no points $\lambda_{k}$, we remove it from the union. Let $B_{m}, m \geq 1$, be all connected components of $B$. We show that the $B_{m}$ are the required sets. By construction, they are mutually disjoint, each containing at least one $\lambda_{k}$. Next, any point $\lambda_{k}$ not belonging to $B(0,1)$ lies in some disk $B\left(z_{p, l}, \alpha\left|z_{p, l}\right|\right)$. Then it lies in $B^{p, l}$ and, therefore, in some $B_{m}$. Thus, we have proved property 1$)$. Now, we verify 2$)$. Let $p \geq 1$, and let $l=0, \ldots, l_{0}$. For every $\lambda_{k} \in B\left(z_{p, l}, 8 \alpha\left|z_{p, l}\right|\right)$ we have $\left|\lambda_{k}\right|<(1+8 \alpha)\left|z_{p, l}\right|<$ $4\left|z_{p, l}\right| / 3$. By the Cartan lower estimate for polynomials (see, e.g., [1, Chapter I, $\S 4$, Theorem 4.1]), for every $H>0$ we have

$$
\ln \left|q_{\Lambda}\left(z, z_{p, l}, 8 \alpha\right)\right| \geq M_{\Lambda}\left(z_{p, l}, 8 \alpha\right) \ln \left(H / 32 e \alpha\left|z_{p, l}\right|\right)
$$

outside of certain exceptional disks $B_{p, l, i}$ with sum of radii of at most $2 H$. Put $H=$ $\alpha\left|z_{p, l}\right| /\left(2\left(l_{0}+1\right)\right)$. Since, for fixed $p$, the sum of diameters of all $B_{p, l, i}$ is $\alpha\left|z_{p, l}\right| / 2$, there exist $\tau_{p}, \widetilde{\tau}_{p} \in(1,2)$ such that the circle $\partial B\left(z_{p, l}, \alpha \tau_{p}\left|z_{p, l}\right|\right)$ does not intersect $B_{p, l, i}$ for every $i$, and the circle $\partial B\left(0, R_{p}\right)$ with $R_{p}=(1+\alpha)^{p-1}\left(1+\alpha \widetilde{\tau}_{p}\right)$ does not intersect $B_{p, l, i}$ for any $l$ and $i$. By (2.12), it follows that

$$
\ln \left|q_{\Lambda}\left(z, z_{p, l}, 8 \alpha\right)\right| \geq-\widetilde{a}\left|z_{p, l}\right|, \quad z \in \partial B\left(z_{p, l}, \alpha \tau_{p}\left|z_{p, l}\right|\right) \cup \partial B\left(0, R_{p}\right) .
$$

By (2.1), this inequality is also fulfilled for $q_{\Lambda}\left(z, z_{p, l}, 3 \alpha\right)$. Taking the definition of $B^{p, l}$ into account, we deduce that

$$
B^{p, l} \subset B\left(z_{p, l}, \alpha \tau_{p}\left|z_{p, l}\right|\right), \quad p=1,2, \ldots, \quad l=0, \ldots, l_{0} .
$$

Let $B_{m}$ intersect a disk $B\left(z_{p, l}, \alpha\left|z_{p, l}\right|\right)$ that contains no points of $B(0,1)$. We show that $B_{m} \subset B\left(z_{p, l}, \alpha \tau_{p, l}\left|z_{p, l}\right|\right)$. Suppose the contrary: there exists $z \in B_{m} \cap \partial B\left(z_{p, l}, \alpha \tau_{p}\left|z_{p l}\right|\right)$. Then $z \in B^{j, s}$ for some $j$ and $s$. By (2.14), we have

Consequently,

$$
z \in B\left(z_{j, s}, \alpha \tau_{j}\left|z_{j, s}\right|\right) \cap \partial B\left(z_{p, l}, \alpha \tau_{p}\left|z_{p, l}\right|\right) .
$$

$$
(1-2 \alpha)\left|z_{j, s}\right| \leq|z| \leq(1+2 \alpha)\left|z_{p, l}\right|, \quad(1-2 \alpha)\left|z_{p, l}\right| \leq|z| \leq(1+2 \alpha)\left|z_{j, s}\right| .
$$

Therefore, for every $w \in B\left(z_{j, s}, 3 \alpha\left|z_{j, s}\right|\right)$ we obtain

$\left|w-z_{p, l}\right| \leq|w-z|+\left|z-z_{p, l}\right|<\alpha\left(5\left|z_{j, s}\right|+2\left|z_{p, l}\right|\right) \leq \alpha\left(\frac{5(1+2 \alpha)}{(1-2 \alpha)}+2\right)\left|z_{p, l}\right|<8 \alpha\left|z_{p, l}\right|$.

This means that $B\left(z_{j, s}, 3 \alpha\left|z_{j, s}\right|\right) \subset B\left(z_{p, l}, 8 \alpha\left|z_{p, l}\right|\right)$. Using (2.1), (2.13), and (2.15), we deduce that

$$
\ln \left|q_{\Lambda}\left(z, z_{j, s}, 3 \alpha\right)\right| \geq \ln \left|q_{\Lambda}\left(z, z_{p, l}, 8 \alpha\right)\right| \geq-\widetilde{a}\left|z_{p, l}\right| \geq-2 \widetilde{a}\left|z_{j, s}\right|,
$$

i.e., $x \notin B^{j, s}$, a contradiction. Thus, we have $B_{m} \subset B\left(z_{p, l}, \alpha \tau_{p, l}\left|z_{p, l}\right|\right)$ for the components indicated above. In particular,

$$
\sup _{z \in B_{m}} d_{m} /|z| \leq 4 \alpha(1-2 \alpha)^{-1} \leq 5 \alpha<d, \quad m \geq m_{0}
$$


This yields the first part of property 2). The second part of it is fulfilled by construction.

Finally, we prove property 3). Let $a=4 \widetilde{a}$. We fix $\delta \in(0, \alpha / 3), w \in \mathbb{C} \backslash B(0,2)$, and $z \in \partial B_{m} \cap B(w, \delta|w|)$. Since the disks $B(w, \delta|w|)$ and $B(0,1)$ do not intersect, there exist indices $p$ and $l$ with $z \in \partial B^{p, l}$. By (2.14), also $z \in B\left(z_{p, l}, 2 \alpha\left|z_{p, l}\right|\right)$. Then, as above, we obtain the inclusion $B(w, \delta|w|) \subset B\left(z_{p, l}, 3 \alpha\left|z_{p, l}\right|\right)$ and, with it, the inequalities

$$
\ln \left|q_{\Lambda}(z, w, \delta)\right| \geq \ln \left|q_{\Lambda}\left(z, z_{p, l}, 3 \alpha\right)\right| \geq-2 \widetilde{a}\left|z_{p, l}\right| \geq-a|z| .
$$

Now, let $z \in \partial B\left(0, R_{p}\right) \cap B(w, \delta|w|)$. By construction, the point $\widetilde{z}=(1+\alpha)^{p-1} z /|z|$ belongs to some disk $B\left(z_{p, l}, \alpha\left|z_{p, l}\right|\right)$. Then it can easily be observed that $B(w, \delta|w|) \subset$ $B\left(z_{p, l}, 8 \alpha\left|z_{p, l}\right|\right)$. Taking (2.1) and (2.13) into account, we obtain

$$
\ln \left|q_{\Lambda}(z, w, \delta)\right| \geq \ln \left|q_{\Lambda}\left(z, z_{p, l}, 8 \alpha\right)\right| \geq-\widetilde{a}\left|z_{p, l}\right| \geq-a|z| .
$$

This proves the lemma.

Theorem 2.1. Suppose that $\Lambda=\left\{\lambda_{k}, n_{k}\right\}_{k=1}^{\infty}$ has finite upper density $N(\Lambda)$. Then for every $d>0$ there exists a partition $U$ of $\Lambda$ such that $\mathcal{S}_{\Lambda}(U)>-\infty, \mathcal{N}(\Lambda, U)<+\infty$, and $\mathcal{D}(\Lambda, U)<d$. Conversely, if there exists a partition $U$ such that $\mathcal{S}_{\Lambda}(U)>-\infty$ and $\mathcal{N}_{1}(\Lambda, U)<+\infty$, then $N(\Lambda)<+\infty$.

Proof. Let $N(\Lambda)<+\infty$. Applying Lemmas 2.5 and 2.4, we find a partition $U$ of $\Lambda$ with $\mathcal{S}_{\Lambda}(U)>-\infty$ and $\mathcal{D}(\Lambda, U)<d$. As was mentioned before Lemma 2.2, moreover, we have $\mathcal{N}(\Lambda, U)<(1+\mathcal{D}(\Lambda, U)) N(\Lambda)$. Conversely, suppose that there exists a partition $U$ with $\mathcal{S}_{\Lambda}(U)>-\infty$ and $\mathcal{N}_{1}(\Lambda, U)<+\infty$. Then, by Lemma 2.2 , the quantity $M_{\Lambda}$ is also finite. Then $N(\Lambda)$ is finite by Lemma 2.1.

\section{§3. Relatively SMALl GROUPS}

In this section, we provide some conditions on the sequence $\Lambda$ that ensure the existence of its partitions into mutually separated relatively small groups.

Let $\alpha>\delta>0$. Consider the functions

$$
\tilde{q}_{\Lambda}(z, w, \alpha, \delta)=\prod_{\lambda_{k} \in B(w, \alpha|w|) \backslash B(w, \delta|w|)}\left(\frac{z-\lambda_{k}}{3 \alpha\left|\lambda_{k}\right|}\right)^{n_{k}} .
$$

If the annulus $B(w, \alpha|w|) \backslash B(w, \delta|w|)$ does not contain any $\lambda_{k}$, we put $\widetilde{q}_{\Lambda} \equiv 1$. Observe that the function $\ln \left|\widetilde{q}_{\Lambda}(z, w, \alpha, \delta)\right|$ is monotone nonincreasing as a function of $\alpha \in(0,1 / 3)$ and monotone nondecreasing as a function of $\delta \in(0, \alpha)$. For a cone $\Gamma$ with vertex at the origin, we define the quantities

$$
S_{\Lambda, \Gamma}^{1}(\alpha)=\lim _{\delta \rightarrow 0} \liminf _{w \in \Gamma,|w| \rightarrow \infty} \frac{\ln \left|\widetilde{q}_{\Lambda}(w, w, \alpha, \delta)\right|}{|w|}, \quad S_{\Lambda, \Gamma}^{1}=\lim _{\alpha \rightarrow 0} S_{\Lambda, \Gamma}^{1}(\alpha) .
$$

They are nonpositive for the same reasons as $\mathcal{S}_{\Lambda}(U)$. We also put

$$
M_{\Lambda, \Gamma}^{0}=\int_{0}^{1} \frac{M_{\Lambda, \Gamma}(\delta)}{\delta} d \delta, \quad M_{\Lambda, \Gamma}^{1}=\liminf _{\delta \rightarrow 0} \ln \delta M_{\Lambda, \Gamma}(\delta) .
$$

If $\Gamma$ is the plane or a ray, we apply the same notational agreements as before. Also, for every $\alpha$ we put

$$
S_{\Lambda, \Gamma}^{2}(\alpha)=\inf _{\xi \in \partial B(0,1) \cap \Gamma} S_{\Lambda, \xi}^{1}(\alpha), \quad S_{\Lambda, \Gamma}^{2}=\inf _{\xi \in \partial B(0,1) \cap \Gamma} S_{\Lambda, \xi}^{1} .
$$

Note that the functions $S_{\Lambda, \xi}^{1}(\alpha)$ and $S_{\Lambda, \Gamma}^{2}$ are monotone nonincreasing for $\alpha \in(0,1 / 3)$. 
Theorem 3.1. Let $\Lambda=\left\{\lambda_{k}, n_{k}\right\}$, and let $\Gamma$ be a cone with vertex at zero. The following statements hold true.

1) If $\Gamma$ is a closed cone and $S_{\Lambda, \Gamma}^{2}(\alpha)>-\infty$ for some $\alpha \in(0,1 / 3)$, then $M_{\Lambda, \Gamma}^{1} \geq$ $S_{\Lambda, \Gamma}^{2}(\alpha)$.

2) If $M_{\Lambda, \Gamma}^{0}<\infty$, then $S_{\Lambda, \Gamma}^{1}=0$.

Proof. 1) Fix $\varepsilon>0$ and choose $\delta \in(0, \alpha / 3)$ with

$$
(1+2 \delta)^{-1}\left(1-\frac{\ln (\alpha(1-3 \delta))}{\ln \delta}\right)\left(S_{\Lambda, \Gamma}^{2}(\alpha)-2 \varepsilon\right)<-S-\varepsilon .
$$

Suppose that $M_{\Lambda, \Gamma}^{1} \leq-S-3 \varepsilon$. Then there exists a sequence $\left\{z_{p}\right\} \subset \Gamma$ with $\left|z_{p}\right| \rightarrow \infty$ such that

$$
\ln \delta M_{\Lambda}\left(z_{p}, \delta\right) /\left|z_{p}\right| \leq S_{\Lambda, \Gamma}^{2}(\alpha)-2 \varepsilon, \quad p \geq 1 .
$$

Suppose the sequence $\left\{z_{p} /\left|z_{p}\right|\right\}$ converges to $\xi \in \partial B(0,1) \cap \Gamma$ and $\left|\xi-z_{p} /\right| z_{p}||<\delta$, $p \geq 1$. For $p \geq 1$, let $w_{p}$ be the point of intersection of the ray $\{t \xi, t \geq 0\}$ and the circle $\partial B\left(z_{p}, 2 \delta\left|z_{p}\right|\right)$ with $\left|w_{p}\right| \geq\left|z_{p}\right|$. Then

$$
B\left(z_{p}, \delta\left|z_{p}\right|\right) \subset\left(B\left(w_{p}, 3 \delta\left|w_{p}\right|\right) \backslash B\left(w_{p}, \widetilde{\delta}\left|w_{p}\right|\right)\right),
$$

where $\widetilde{\delta}=\delta(1+2 \delta)^{-1}$. Since the summands of the series expansion for $\ln \left|\widetilde{q}_{\Lambda}\right|$ are positive, by (3.2) and (3.1) it follows that

$$
\begin{aligned}
& \frac{\ln \left|\widetilde{q}_{\Lambda}\left(w_{p}, w_{p}, \alpha, \widetilde{\delta}\right)\right|}{\left|w_{p}\right|} \leq \frac{1}{\left|w_{p}\right|} \sum_{\lambda_{k} \in B\left(z_{p}, \delta\left|z_{p}\right|\right)} n_{k} \ln \frac{\left|w_{p}-\lambda_{k}\right|}{3 \alpha\left|\lambda_{k}\right|} \\
& \leq \frac{1}{(1+2 \delta)\left|z_{p}\right|} \sum_{\lambda_{k} \in B\left(z_{p}, \delta\left|z_{p}\right|\right)} n_{k} \ln \frac{\delta}{\alpha(1-3 \delta)}<S_{\Lambda, \Gamma}^{2}(\alpha)-\varepsilon, \quad p \geq 1 .
\end{aligned}
$$

Since $\ln \left|\widetilde{q}_{\Lambda}\left(w_{p}, w_{p}, \alpha, \delta^{\prime}\right)\right|$ is monotone nondecreasing as a function of $\delta^{\prime}$, we have

$$
\liminf _{p \rightarrow \infty} \ln \left|\widetilde{q}_{\Lambda}\left(w_{p}, w_{p}, \alpha, \widetilde{\delta}\right)\right| /\left|w_{p}\right| \geq S_{\Lambda, \xi}^{1}(\alpha) \geq S_{\Lambda, \Gamma}^{2}(\alpha) .
$$

This contradicts (3.3). Thus, $M_{\Lambda, \Gamma}^{1} \geq S_{\Lambda, \Gamma}^{2}(\alpha)$, and statement 1) is proved.

We prove statement 2). Suppose that $M_{\Lambda, \Gamma}^{0}<\infty$ and $\varepsilon>0$. Then there exists $\alpha_{0} \in(0,1 / 3)$ such that for all $0<\delta<\alpha<\alpha_{0}$ we have

$$
\int_{\beta}^{\alpha} \frac{M_{\Lambda, \Gamma}(\tilde{\delta})}{\widetilde{\delta}} d \tilde{\delta} \leq \varepsilon .
$$

Fixing positive numbers $\delta<\alpha<\alpha_{0}$, we choose a natural number $s(\delta, \alpha)$ such that for $h=(\alpha-\delta) / s(\delta, \alpha) \leq \delta$ we have

$$
\sum_{s=1}^{s(\delta, \alpha)} \frac{M_{\Lambda, \Gamma}(\delta+s h)}{\delta+s h} h-\int_{\delta}^{\alpha} \frac{M_{\Lambda, \Gamma}(\widetilde{\delta})}{\widetilde{\delta}} d \widetilde{\delta} \leq \varepsilon .
$$

Find $R>0$ such that

$$
\begin{array}{r}
M_{\Lambda}(w, \delta+s h) \leq\left(M_{\Lambda, \Gamma}(\delta+s h)+\varepsilon / \ln (\alpha / \delta)\right)|w|, \\
s=1, \ldots, s(\delta, \alpha), w \in \Gamma \backslash B(0, R) .
\end{array}
$$


Taking (3.5) and (3.4) into account, we obtain

$$
\begin{aligned}
& \frac{1}{|w|} \int_{\delta|w|}^{\alpha|w|} \frac{M_{\Lambda}(w, t /|w|)}{t} d t=\frac{1}{|w|} \int_{\delta}^{\alpha} \frac{M_{\Lambda}(w, \widetilde{\delta})}{\widetilde{\delta}} d \widetilde{\delta} \\
& \quad \leq \frac{1}{|w|} \sum_{s=1}^{s(\delta, \alpha)} \int_{\delta+(s-1) h}^{\delta+s h} \frac{M_{\Lambda}(w, \delta+s h)}{\widetilde{\delta}} d \widetilde{\delta} \\
& \quad \leq \sum_{s=1}^{s(\delta, \alpha)}\left(\frac{M_{\Lambda, \Gamma}(\delta+s h)}{\delta+(s-1) h} h+\int_{\delta+(s-1) h}^{\delta+s h} \frac{\varepsilon / \ln (\alpha / \delta)}{\widetilde{\delta}} d \widetilde{\delta}\right) \leq 5 \varepsilon, \quad w \in \Gamma \backslash B(0, R) .
\end{aligned}
$$

We put $g(t)=0, t \in(0, \delta|w|), g(t)=\left(M_{\Lambda}(w, t /|w|)-M_{\Lambda}(w, \delta)\right) / t, t \in[\delta|w|, \alpha|w|)$, and $g(t)=\left(M_{\Lambda, \Gamma}(z, \alpha)-M_{\Lambda, \Gamma}(w, \delta 0) / t, t \geq \alpha|w|\right.$. The above calculations show that

$$
\begin{aligned}
\frac{1}{|w|} \int_{0}^{5 \alpha|w|} g(t) d t & =\frac{1}{|w|} \int_{\delta|w|}^{\alpha|w|} \frac{M_{\Lambda}(w, t /|w|)-M_{\Lambda}(w, \delta)}{t} d t+\frac{\left(M_{\Lambda}(w, \alpha)-M_{\Lambda}(w, \delta)\right) \ln 5}{|w|} \\
& \leq 5 \varepsilon+M_{\Lambda}(w, \alpha) \ln 5 /|w|, \quad w \in \Gamma \backslash B(0, R) .
\end{aligned}
$$

Since $\ln \left|\widetilde{q}_{\Lambda}(z, w, \alpha, \delta)\right| \geq 0$ for $z \in \partial B(w, 5 \alpha|w|)$, the Jensen formula yields

$$
\begin{aligned}
\frac{\ln \left|\widetilde{q}_{\Lambda}(w, w, \alpha, \delta)\right|}{|w|} & =\frac{1}{2 \pi|w|} \int_{0}^{2 \pi} \ln \left|\widetilde{q}_{\Lambda}(w+5 \alpha|w| \exp (i \theta), w, \alpha, \delta)\right| d \theta-\frac{1}{|w|} \int_{0}^{5 \alpha|w|} g(t) d t \\
& \geq-5 \varepsilon-M_{\Lambda}(w, \alpha) \ln 5 /|w|, \quad w \in \Gamma \backslash B(0, R) .
\end{aligned}
$$

It follows that $S_{\Lambda, \Gamma}^{1} \geq-M_{\Lambda, \Gamma} \ln 5$. Since $M_{\Lambda, \Gamma}^{0}<\infty$, we have $M_{\Lambda, \Gamma}=0$. Therefore, $S_{\Lambda, \Gamma}^{1}=0$, which completes the proof of the theorem.

Remarks. 1. If $S_{\Lambda, \Gamma}^{1}>-\infty$, then for every $\varepsilon>0$ there exists $\alpha \in(0,1 / 3)$ with $S_{\Lambda, \Gamma}^{1}(\alpha) \geq$ $S_{\Lambda, \Gamma}^{1}-\varepsilon$. In particular, $S_{\Lambda, \xi}^{1}(\alpha) \geq S_{\Lambda, \Gamma}^{1}-\varepsilon, \xi \in \partial B(0,1) \cap \Gamma$. Then $M_{\Lambda, \Gamma}^{1} \geq S_{\Lambda, \Gamma}^{1}-\varepsilon$ by Theorem 3.1. Since $\varepsilon>0$ is arbitrary, we obtain $M_{\Lambda, \Gamma}^{1} \geq S_{\Lambda, \Gamma}^{1}$. It can be shown that the last inequality is also true for not necessarily closed angles $\Gamma$.

2. The converse to statement 1 ) of Theorem 3.1 is not true. Moreover, the condition $M_{\Lambda, \Gamma}^{1}>-\infty$ does not even imply that the quantities $S_{\Lambda, \xi}^{1}(\alpha)$ are finite. We consider the corresponding example. Let $\Lambda=\bigcup_{p, l=1}^{\infty, p} \Lambda_{p, l}$, where $\Lambda_{p, p}=\left\{2^{p}\right\}$, and

$$
\begin{aligned}
\Lambda_{p, l} & =\left\{2^{p}\left(1-\exp \left(-2^{l+1}\right)\right)-s h_{l}, s=0,1, \ldots, 2^{p-l}-1\right\}, \\
h_{l} & =\left(\exp \left(-2^{l}\right)-\exp \left(-2^{l+1}\right)\right) 2^{l}
\end{aligned}
$$

for $l<p$. We show that $M_{\Lambda}^{1}>-\infty$. Fix $\delta \in\left(0,3^{-1} \exp (-2)\right)$ and choose $p_{0}$ with $\exp \left(-2^{p_{0}}\right)<3 \delta$. Let $|z|>(1-\delta)^{-1} 2^{p_{0}}$, and let the interval $V=B(z, \delta|z|) \cap \mathbb{R}$ contain a point of $\Lambda_{p, l}$. Then $p>p_{0}$ and $(1+\delta)^{-1}(1-\exp (-2)) 2^{p}<|z|<(1-\delta)^{-1} 2^{p}$. Consequently, the length of $V$ is of at most $2 \delta(1-\delta)^{-1} 2^{p}<3 \delta 2^{p}$, and this interval has no points in common with $\Lambda_{s, j}$ if $s \neq p$. Since $\exp \left(-2^{p}\right)<h_{p, p-1}<\cdots<h_{p, 1}$, we see that the number of points of $\Lambda$ that lie in $V$ does not exceed the number of points of $\Lambda$ that lie in the half-open interval $\left((1-3 \delta) 2^{p}, 2^{p}\right]$. We choose an index $l=1, \ldots, p-1$ such that $\exp \left(-2^{l+1}\right) \leq 3 \delta \leq \exp \left(-2^{l}\right)$. Then we have

$$
\ln \delta \frac{M_{\Lambda}(z, \delta)}{|z|} \geq \ln \left(\frac{\exp \left(-2^{l+1}\right)}{3}\right) \frac{M_{\Lambda}\left(2^{p}, \exp \left(-2^{l}\right)\right)}{(1+\delta)^{-1} 2^{p-1}}=\frac{\left(-2^{l+1}-\ln 3\right) 2^{p-l+1}}{(1+\delta)^{-1} 2^{p-1}} \geq-32 .
$$


Thus, $M_{\Lambda}^{1} \geq-32$. Now, we show that $S_{\Lambda}^{1}=-\infty$. Put $a_{j}=\exp \left(-2^{j}\right), \delta_{m}=\exp \left(-2^{m}\right)$, and let $p>m>j$. Then

$$
\begin{aligned}
& \frac{\ln \left|\widetilde{q}_{\Lambda}\left(2^{p}, 2^{p}, \alpha_{j}, \delta_{m}\right)\right|}{2^{p}}=\frac{1}{2^{p}} \sum_{l=j}^{m-1} \sum_{\lambda_{k} \in \Lambda_{p, l}} \ln \frac{\left|2^{p}-\lambda_{k}\right|}{3 \alpha_{j}\left|\lambda_{k}\right|} \leq \sum_{l=j}^{m-1} \frac{1}{2^{l}} \ln \frac{\exp \left(-2^{l}\right) 2^{p}}{3 \alpha_{j}\left|\lambda_{k}\right|} \\
& \leq \sum_{l=j}^{m-1} \frac{1}{2^{l}} \ln \frac{\exp \left(-2^{l}\right)}{3 \alpha_{j}\left(1-\exp \left(-2^{j}\right)\right)}=-(m-1-j)+\sum_{l=j}^{m-1} \frac{1}{2^{l-j}} \leq-(m-1-j)+2 .
\end{aligned}
$$

It follows that $S_{\Lambda}^{1}\left(\alpha_{j}\right)=-\infty$. Therefore, $S_{\Lambda}^{1}=-\infty$.

3. The converse to statement 2 of Theorem 3.1 also fails. To show this, we need to slightly modify the preceding example.

In a standard way, we enumerate the pairs $\{(l, j)\}_{l, j=1}^{\infty}$ of positive integers by indices starting with 2 , and denote by $p(l, j)$ the index of the pair $(l, j)$. Thus, $p(1,1)=2$, $p(1,2)=3, p(2,1)=4$, and so on. Let $\Lambda=\bigcup_{p=2}^{\infty} \Lambda_{p}$, and let $a_{l} \in[1 / l, 1]$. Denote by $m_{l, j}$ the integral part of $2^{p(l, j)-l}$. For all $p(l, j) \geq 2$, we put

$$
\Lambda_{p(l, j)}=\left\{2^{p(l, j)}\left(1-\exp \left(-2^{l+1}\right)\right)-s h_{l, j}, s=0,1, \ldots, m_{l, j}-1\right\},
$$

where

$$
h_{l, j}=2^{p(l, j)}\left(\exp \left(-2^{l}\right)-\exp \left(-2^{l+1}\right)\right) / m_{l, j} .
$$

It can easily be observed that the quantities $h_{l, j} \geq 0$ have a common upper bound, and that the function $M_{\Lambda}(\delta)$ can only become smaller if $a_{l}$ increases. If $a_{l}=1, l \geq 1$, then $\Lambda$ is part of the sequence from the preceding example. Thus, $M_{\Lambda}^{1} \geq-32$ for every choice of $a_{l} \in[2 / l, 1]$. In particular, $M_{\Lambda}=0$.

Now, we estimate the quantity $S_{\Lambda}$ from below $\left(S_{\Lambda}=S_{\Lambda}(U)\right.$, where $U=\left\{U_{k}\right\}$ is the trivial splitting, i.e., $\left.U_{k}=\left\{\lambda_{k}\right\}, k \geq 1\right)$. Fixing $\varepsilon>0$, we choose $\delta_{0} \in(0,1 / 24)$ with $M_{\Lambda}(\delta)<\varepsilon$ for $\delta \in\left(0, \delta_{0}\right)$. Next, for every $\delta \in\left(0, \delta_{0}\right)$, we find $R(\delta)>0$ satisfying $M_{\Lambda}(z, \delta) /|z|<2 \varepsilon,|z|>R(\delta)$. Let $\lambda_{k}>R(\delta)$, and let $p(l, j)$ be the index with $\lambda_{k} \in$ $\Lambda_{p(l, j)}$. Two cases are possible.

1) $\delta \lambda_{k} \leq 2^{p(l, j)}\left(\exp \left(-2^{l}\right)-\exp \left(-2^{l+1}\right)\right)$. Among the points of $\Lambda$, only those in $\Lambda_{p(l, j)}$ may belong to $B\left(\lambda_{k}, \delta \lambda_{k}\right)$. Thus, the number of points of $\Lambda_{p(l, j)}$ belonging to the interval $\left(\lambda_{k}-\delta \lambda_{k}, \lambda_{k}+\delta \lambda_{k}\right)$ is equal to $M_{\Lambda}\left(\lambda_{k}, \delta\right)$. Let $n$ be the maximal natural number with $n h_{l, j}<\delta \lambda_{k}$. Then $M_{\Lambda}\left(\lambda_{k} \delta\right) \geq n$. Since $n h_{l j} /\left(3 \delta(1+\delta) \lambda_{k}\right)<1$, we have

$$
\begin{aligned}
\frac{\ln \left|q_{\Lambda, U}^{k}\left(\lambda_{k}, \delta\right)\right|}{\lambda_{k}} & =\sum_{\substack{\lambda_{s} \in B\left(\lambda_{k}, \delta\left|\lambda_{k}\right|\right) \\
s \neq k}} \frac{1}{\lambda_{k}} \ln \frac{\left|\lambda_{k}-\lambda_{s}\right|}{3 \delta \lambda_{s}} \geq \frac{1}{\lambda_{k}} \ln \frac{(n !)^{2} h_{j, j}^{2 n}}{\left(3 \delta(1+\delta) \lambda_{k}\right)^{2 n}} \\
& \geq \frac{2 n}{\lambda_{k}} \ln \frac{n h_{l, j}}{9 \delta(1+\delta) \lambda_{k}} \geq 4 \varepsilon \ln \frac{\alpha \lambda_{k}-h_{l, j}}{9 \delta(1+\delta) \lambda_{k}}=4 \varepsilon \ln \frac{1-h_{l j} / \delta \lambda_{k}}{9(1+\delta)} .
\end{aligned}
$$

2) $\delta \lambda_{k}>2^{p(l, j)}\left(\exp \left(-2^{l}\right)-\exp \left(-2^{l+1}\right)\right)$. Since $m_{l, j} h_{l, j} /\left(3 \delta(1+\delta) \lambda_{k}\right)<1$, we have

$$
\begin{aligned}
& \frac{\ln \left|q_{\Lambda, U}^{k}\left(\lambda_{k} \delta\right)\right|}{\lambda_{k}}=\sum_{\substack{\lambda_{s} \in B\left(\lambda_{k}, \delta\left|\lambda_{k}\right|\right) \\
s \neq k}} \frac{1}{\lambda_{k}} \ln \frac{\left|\lambda_{k}-\lambda_{s}\right|}{3 \delta \lambda_{s}} \geq \frac{1}{\lambda_{k}} \ln \frac{\left(m_{l, j} !\right)^{2} h^{2 m_{j, j}}}{\left(3 \delta(1+\delta) \lambda_{k}\right)^{2 m_{l, j}}} \\
& \geq \frac{2 m_{l, j}}{\lambda_{k}} \ln \frac{m_{l, j} h_{l, j}}{9 \delta(1+\delta) 2^{p(l, j)}}=\frac{2 m_{l, j}}{\lambda_{k}} \ln \frac{2^{p(l, j)}\left(\exp \left(-2^{l}\right)-\exp \left(-2^{l+1}\right)\right)}{9 \delta(1+\delta) 2^{p(l, j)}} \\
& =\frac{2 m_{l, j}}{\lambda_{k}}\left(-2^{l}+\ln \frac{\left(1-\exp \left(-2^{l}\right)\right)}{2 \delta(1+\delta)}\right) \geq \frac{2 m_{l, j}}{\lambda_{k}}\left(-2^{l}-\ln (24 \delta)\right) \geq-\frac{2^{l+1} m_{l, j}}{2^{p(l, j)}(1-\exp (-2))} .
\end{aligned}
$$


Note that $h_{l, j} / \delta \lambda_{k} \rightarrow 0$ as $k \rightarrow \infty$, and $p(l, j)-l \rightarrow \infty$ as $p(l, j) \rightarrow \infty$. Therefore, in both cases we obtain

$$
\liminf _{k \rightarrow \infty} \ln \left|q_{\Lambda, U}^{k}\left(\lambda_{k}, \delta\right)\right| / \lambda_{k} \geq-4 \varepsilon \ln (2(1+\delta))-3 \sup _{1 \geq l(\delta)} a_{l} .
$$

In follows that

$$
\mathcal{S}_{\Lambda}=\mathcal{S}_{\Lambda}(U)=\lim _{\delta \rightarrow 0} \liminf _{k \rightarrow \infty} \ln \left|q_{\Lambda, U}^{k}\left(\lambda_{k}, \delta\right)\right| / \lambda_{k} \geq-4 \varepsilon \ln 9-3 \limsup _{l \rightarrow \infty} a_{l} .
$$

Since $\varepsilon>0$ is arbitrary, we have $\mathcal{S}_{\Lambda} \geq-3 \lim \sup _{l \rightarrow \infty} a_{l}$. It will be proved in Theorem 3.2 that $S_{\Lambda}^{1} \geq 2 \mathcal{S}_{\Lambda}$. Therefore, $S_{\Lambda}^{1} \geq-6 \lim \sup _{l \rightarrow \infty} a_{l}$. By the arguments in the first example, it is easy to deduce that $S_{\Lambda}^{1} \leq-\lim \sup _{l \rightarrow \infty} a_{l}$. Thus, the vanishing of $S_{\Lambda}^{1}$ is determined by the numbers $a_{l}$. Let $a_{l}=1 / l$. Then $S_{\Lambda}^{1}=0$. We show that $M_{\Lambda}^{0}=\infty$ despite of this. We have

$$
M_{\Lambda}\left(\exp \left(-2^{l}\right)\right) \geq \limsup _{j \rightarrow \infty} 2^{-p(l, j)} M_{\Lambda}\left(2^{p(l, j)}, \exp \left(-2^{l}\right)\right)=2^{-l} l^{-1}, \quad l \geq 1 .
$$

Consequently,

$$
M_{\Lambda}\left(\delta_{l}\right) \geq-\ln 2 /\left(\ln \delta_{l} \ln \left|\ln \delta_{l}\right|\right), \quad \delta_{l}=\exp \left(-2^{l}\right), \quad l \geq 1 .
$$

Since the function $M_{\Lambda}(\delta)$ is monotone nondecreasing, we deduce that

$$
M_{\Lambda}(\delta) \geq M_{\Lambda}\left(\delta_{l+1}\right) \geq-\frac{\ln 2}{\ln \delta_{l+1} \ln \left|\ln \delta_{l+1}\right|} \geq-\frac{\ln 2}{4 \ln \delta_{l} \ln \left|\ln \delta_{l}\right|} \geq \frac{\ln 2}{4 \ln \delta \ln |\ln \delta|}
$$

for all $\delta \in\left(\delta_{l+1} \delta_{l}\right)$. This yields the required relation $M_{\Lambda}^{0}=\infty$.

We are going to indicate some conditions on the sequence $\Lambda$ under which it admits a splitting $U$ into relatively small groups with $\mathcal{S}_{\Lambda}(U)>-\infty$. But before we prove several auxiliary statements.

Lemma 3.1. Let $\Lambda=\left\{\lambda_{k}, n_{k}\right\}$. Suppose that $S_{\Lambda}^{2}(\widetilde{\alpha})>-\infty$ for some $\widetilde{\alpha} \in(0,1 / 3)$. Then $M_{\Lambda}^{1}>-\infty$ and there exist open sets $B_{m}, m \geq 1$, with the following properties.

1) The sets $B_{m}, m \geq 1$, are mutually disjoint, their union contains all $\lambda_{k}$, and each $B_{m}$ contains at least one $\lambda_{k}$.

2) The diameters $d_{m}$ of the sets $B_{m}$ satisfy the condition

$$
\limsup _{m \rightarrow \infty} \sup _{z \in B_{m}} d_{m} /|z|=0 .
$$

3) For every $\varepsilon>0$ and $\alpha \in(0, \widetilde{\alpha}]$ there exists $R>0$ with

$$
\begin{aligned}
& \ln \left|q_{\Lambda}(z, w, \alpha / 6)\right| \geq\left(S_{\Lambda}^{2}(\alpha)+M_{\Lambda}^{1}-M_{\Lambda}(\alpha) \ln 3-\varepsilon\right)|z|, \\
& z \in \partial B_{m} \cap B(w, \alpha|w| / 6), \quad m \geq 1, \quad|w| \geq R .
\end{aligned}
$$

Proof. Theorem 3.1 shows that $M_{\Lambda}^{1} \geq S_{\Lambda}^{2}(\widetilde{a})>-\infty$. Therefore, $M_{\Lambda}=0$. By the definition of $M_{\Lambda}$ and $M_{\Lambda}^{1}$, for every $p \geq 1$ we can find $\delta_{p} \in(0, \tilde{\alpha} / 8 p)$ such that

$$
\begin{aligned}
& \ln (256 e \widetilde{\alpha}) \limsup _{|w| \rightarrow \infty} M_{\Lambda}\left(w, 8 \delta_{p}\right) /|w| \leq 1 / p, \\
& \ln \left(8 \delta_{p}\right) \limsup _{|w| \rightarrow \infty} M_{\Lambda}\left(w, 8 \delta_{p}\right) /|w| \geq M_{\Lambda}^{1}-1 / p .
\end{aligned}
$$

Since $\ln \left|\widetilde{q}_{\Lambda}(w, w, \alpha, \beta)\right|$ is monotone nondecreasing with respect to $\delta$, for every $\xi \in$ $\partial B(0,1)$ we have

$$
\liminf _{t \rightarrow+\infty} \ln \left|\widetilde{q}_{\Lambda}\left(t \xi, t \xi, \widetilde{\alpha} / s, 3 \delta_{p}\right)\right| / t \geq S_{\Lambda, \xi}^{1}(\widetilde{\alpha} / s) \geq S_{\Lambda}^{2}(\widetilde{\alpha} / s), \quad s=1, \ldots, p .
$$


Choose $\varphi_{p} \in(0, \pi / 2)$ such that the set $\left\{z=r e^{i \tau}: \tau \in\left[-\varphi_{l} / 2, \varphi_{p} / 2\right], r \geq 1\right\}$ is included in the union of the disks $B\left(\left(1+\delta_{p}\right)^{\mu}, \delta_{p}\left(1+\delta_{p}\right)^{\mu}\right), \mu=0,1, \ldots$ Let $l_{p}$ denote the maximal natural number $l$ with $l \varphi_{p}<2 \pi$. We choose $R_{p}>p$ in such a way that

$$
\begin{aligned}
& \ln \left|\widetilde{q}_{\Lambda}\left(t e^{i l \varphi_{p}}, t e^{i l \varphi_{p}}, \widetilde{\alpha} / s, 3 \delta_{p}\right)\right| \geq\left(S_{\Lambda}^{2}(\widetilde{\alpha} / s)-2 / p\right) t, \quad l=\overline{0, l_{p}}, \quad s=\overline{1, p}, \quad t \geq R_{p} \\
& \ln (256 e \widetilde{\alpha}) \frac{M_{\Lambda}\left(w, 8 \delta_{p}\right)}{|w|} \leq \frac{2}{p}, \quad \ln \left(8 \delta_{p}\right) \frac{M_{\Lambda}\left(w, 8 \delta_{p}\right)}{|w|} \geq\left(M_{\Lambda}^{1}-\frac{2}{p}\right), \quad|w| \geq R_{p} .
\end{aligned}
$$

We may assume, that, moreover,

$$
\delta_{p+1} \leq \delta_{p}, \quad\left(1+2 \delta_{p}\right) R_{p+1} \leq \frac{\left(1-2 \delta_{p+1}\right)^{2}}{\left(1+2 \delta_{p+1}\right)} R_{p+2}, \quad R_{p+1}=\left(1+\delta_{p}\right)^{\mu_{p}} R_{p},
$$

where $\mu_{p}$ is some positive integer. Put $z_{p, l, \mu}=R_{p}\left(1+\delta_{p}\right)^{\mu} e^{i l \varphi_{p}}, p \geq 1, l=0, \ldots, l_{p}, \mu=$ $0, \ldots, \mu_{p}$. By the identity in $(3.8)$, the union of $B\left(0, R_{1}\right)$ and the disks $B\left(z_{p, l, \mu}, \delta_{p}\left|z_{p, l, \mu}\right|\right)$ covers the plane. For every $p, l, \mu$, and $b=8-M_{\Lambda}^{1}$, consider the set

$$
\widetilde{B}^{p, l, \mu}=\left\{z \in \mathbb{C}: \sum_{\lambda_{k} \in B\left(z_{p, l, \mu}, 3 \delta_{p}\left|z_{p, l, \mu}\right|\right)} n_{k} \ln \frac{\left|z-\lambda_{k}\right|}{3 \widetilde{\alpha}\left|\lambda_{k}\right|}<\left(M_{\Lambda}^{1}-b / p\right)\left|z_{p, l, \mu}\right|\right\} .
$$

Let $B^{p, l, \mu}$ be the union of all its connected components that intersect the disk

$$
B\left(z_{p, l, \mu}, \delta_{p}\left|z_{p, l, \mu}\right|\right)
$$

and contain at least one of the points $\lambda_{k}$. Put

$$
B=\bigcup_{p=1, l=0, \mu=0}^{\infty, l(p), s(p)} B^{p, l, \mu} \cup B\left(0, R_{1}\right) .
$$

If $B\left(0, R_{1}\right)$ does not contain any of the $\lambda_{k}$, we remove this disk from the union. Denote by $B_{m}, m \geq 1$, all connected components of $B$. We show that $B_{m}$ is the required sequence of sets. Property 1 ) is satisfied for the same reasons as in Lemma 2.5. We verify property 2 ). Fixing $p, l$, and $\mu$, we argue as in Lemma 2.5 to obtain the following inequality with the help of the Cartan theorem:

$$
\sum_{\lambda_{k} \in B\left(z_{p, l, \mu}, 8 \delta_{p}\left|z_{p, l, \mu}\right|\right)} n_{k} \ln \frac{\left|z-\lambda_{k}\right|}{3 \widetilde{\alpha}\left|\lambda_{k}\right|} \geq M_{\Lambda}\left(z_{p, l, m}, 8 \delta_{p}\right) \ln \frac{\delta_{p}}{32 e \widetilde{\alpha}},
$$

where $\tau_{p, l, \mu} \in(1,2)$ and $z \in \partial B\left(z_{p, l, \mu}, \tau_{p, l, \mu} \delta_{p}\left|z_{p, l, \mu}\right|\right)$. By (3.7), we have

$$
\begin{aligned}
M_{\Lambda}\left(z_{p, l, \mu}, 8 \delta_{p}\right) \ln \frac{\delta_{p}}{32 e \widetilde{\alpha}} & \geq\left(M_{\Lambda}^{1}-2 / p\right)\left|z_{p, l, \mu}\right|-M_{\Lambda}\left(z_{p, l, \mu}, 8 \delta_{p}\right) \ln (256 e \tilde{\alpha}) \\
& \geq\left(M_{\Lambda}^{1}-4 / p\right)\left|z_{p, l, \mu}\right| .
\end{aligned}
$$

Thus, we arrive at

$$
\begin{array}{r}
\sum_{\lambda_{k} \in B\left(z_{p, l, \mu}, 8 \delta_{p}\left|z_{p, l, \mu}\right|\right)} n_{k} \ln \frac{\left|z-\lambda_{k}\right|}{3 \widetilde{\alpha}\left|\lambda_{k}\right|} \geq\left(M_{\Lambda}^{1}-4 / p\right)\left|z_{p, l, \mu}\right|, \\
z \in \partial B\left(z_{p, l, \mu} \tau_{p, l, \mu} \delta_{p}\left|z_{p, l, \mu}\right|\right) .
\end{array}
$$

As in Lemma 2.5, it follows that

$$
B^{p, l, \mu} \subset B\left(z_{p, l, \mu}, \tau_{p, l, \mu} \delta_{p}\left|z_{p, l, \mu}\right|\right) .
$$

Let $B_{m}$ contain some points outside $B\left(0, R_{3}\right)$, and let $\tilde{z}$ be one of them. We choose the maximal $p$ with $R_{p} \leq|\widetilde{z}|$ and pick a disk $B\left(z_{p, l, \mu}, \delta_{p}\left|z_{p, l, \mu}\right|\right)$ containing $\tilde{z}$. The component $B_{m}$ either is included in $B\left(z_{p, l, \mu}, \tau_{p, l, \mu} \delta_{p}\left|z_{p, l, \mu}\right|\right)$ or contains a point of the circle $\partial B\left(z_{p, l, \mu}, \tau_{p, l, \mu} \delta_{p}\left|z_{p, l, \mu}\right|\right)$. By construction, $z \in B^{j, n, \nu}$ for some $j, n$, and $\nu$ (because $z \notin B\left(0, R_{1}\right)$ by $\left.(3.8)\right)$. Suppose that $j \geq p$. Then $\delta_{j} \leq \delta_{p}$ by (3.8). Therefore, as in 
Lemma 2.5, we have $B\left(z_{j, n, \nu}, 3 \delta_{j}\left|z_{j, n, \nu}\right|\right) \subset B\left(z_{p, l, \mu}, 8 \delta_{p}\left|z_{p, l, \mu}\right|\right)$. Since $8 \delta_{p}<1 / p$, we can argue as in (2.1) with the use of (3.9) and (3.10) to obtain

$$
\begin{aligned}
\sum_{\lambda_{k} \in B\left(z_{j, n, \nu}, 3 \delta_{j}\left|z_{j, n, \nu}\right|\right)} n_{k} \ln \frac{\left|z-\lambda_{k}\right|}{3 \widetilde{\alpha}\left|\lambda_{k}\right|} \geq \sum_{\lambda_{k} \in B\left(z_{p, l, \mu}, 8 \delta_{p}\left|z_{p, l, \mu}\right|\right)} n_{k} \ln \frac{\left|z-\lambda_{k}\right|}{3 \widetilde{\alpha}\left|\lambda_{k}\right|} \\
\geq\left(M_{\Lambda}^{1}-4 / p\right)\left|z_{p, l, \mu}\right| \geq\left(M_{\Lambda}^{1}-4 / p\right) \frac{1+2 \delta_{j}}{1-2 \delta_{p}}\left|z_{j, n, \nu}\right| \\
>\left(M_{\Lambda}^{1}-4 / p+8\left(M_{\Lambda}^{1}-4\right) \delta_{p}\right)\left|z_{p, l, \mu}\right|>\left(M_{\Lambda}^{1}-b / p\right)\left|z_{p, l, \mu}\right| .
\end{aligned}
$$

This contradicts the relation $z \in B^{j, n, \nu}$. Consequently, $j<p$. Since $|z| \geq(1-$ $\left.2 \delta_{p}\right)\left|z_{p, l, \mu}\right| \geq\left(1-2 \delta_{p}\right) R_{p}$, by (3.10) and (3.8) we obtain $j=p-1$. The component $B_{m}$ either is included in $B\left(z_{j, n, \nu}, \tau_{j, n, \nu} \delta_{j}\left|z_{j, n, \nu}\right|\right)$ or contains a point of the circle $\partial B\left(z_{j, n, \nu}, \tau_{j, n, \nu} \delta_{j}\left|z_{j, n, \nu}\right|\right)$. In the second case we have $|y| \geq\left(1+2 \delta_{p-1}\right)^{-1}\left(1-2 \delta_{p-1}\right)^{2} R_{p}$. Therefore, by (3.10) and (3.8), the index $i$ for the set $B^{i, s, \tau}$ that contains $y$ (such set exists because $\left.y \notin B\left(0, R_{1}\right)\right)$ is greater than or equal to $j$. So, we encounter a situation already considered, which yields a contradiction. Thus,

$$
B_{m} \subset B\left(z_{p, l, \mu}, \tau_{p, l, \mu} \delta_{p}\left|z_{p, l, \mu}\right|\right)
$$

for the components $B_{m}$ mentioned above (here $p, l$, and $\mu$ depend on $m$ ). This yields property 2) in the lemma.

Finally, we verify property 3$)$. Fixing $\varepsilon>0$ and $\alpha \in(0, \widetilde{\alpha})$, we choose the minimal number $p_{0}$ with $\widetilde{\alpha} / p_{0} \leq \alpha$. Now, we find $p \geq p_{0}$ with

$$
\begin{gathered}
\frac{b}{j}<\frac{\varepsilon}{4}, \quad \frac{2 \delta_{j}\left(M_{\Lambda}^{1}+S_{\Lambda}^{2}(\alpha)-M_{\Lambda}(\alpha) \ln 3-3 \varepsilon / 4\right)}{1-2 \delta_{j}}>-\frac{\varepsilon}{4}, \\
M_{\Lambda}\left(z_{j, l, \mu}, \widetilde{\alpha} / p_{0}\right) \ln 3 \leq\left(M_{\Lambda}\left(\widetilde{\alpha} / p_{0}\right) \ln 3+\varepsilon / 4\right)\left|z_{j, l, \mu}\right| \\
\leq\left(M_{\Lambda}(\alpha) \ln 3+\varepsilon / 4\right)\left|z_{j, l, \mu}\right|, \quad j \geq p .
\end{gathered}
$$

Put $R=(1-\alpha)^{-1} R_{p+1}$ and suppose that $w \in \mathbb{C} \backslash B(0, R)$ and $z \in \partial B_{m} \cap B(w, \alpha|w| / 6)$. By (3.10) and (3.8), we have $z \in \partial B^{j, l, \mu} \subset B\left(z_{j, l, \mu}, 2 \delta_{j}\left|z_{j, l, \mu}\right|\right)$ for some $j \geq p, l, \mu$. It can easily be seen that $B(w, \alpha|w| / 6) \subset B\left(z_{j, l, \mu}, \tilde{a l}\left|z_{j, l, \mu}\right| / p_{0}\right)$. Then

$$
\left|q_{\Lambda}(z, w, \alpha / 6)\right| \geq \mid q_{\Lambda}\left(z, z_{j, l, \mu}, \widetilde{\alpha} / p_{0} \mid\right.
$$

by (2.1). We estimate $\left|q_{\Lambda}\left(z, z_{j, l, \mu}, \widetilde{a} / p_{0}\right)\right|$ from below. By the definition of $B^{j, l, \mu}$ and the first inequality in (3.11), we have

$$
\begin{aligned}
\sum_{\lambda_{k} \in B\left(z_{j, l, \mu}, 3 \delta_{j}\left|z_{j, l, \mu}\right|\right)} & n_{k} \ln \frac{p_{0}\left|z-\lambda_{k}\right|}{3 \widetilde{\alpha}\left|\lambda_{k}\right|} \\
& \geq \sum_{\lambda_{k} \in B\left(z_{j, l, \mu}, 3 \delta_{j}\left|z_{j, l, \mu}\right|\right)} n_{k} \ln \frac{\left|z-\lambda_{k}\right|}{3 \widetilde{\alpha}\left|\lambda_{k}\right|} \geq\left(M_{\Lambda}^{1}-\frac{\varepsilon}{4}\right)\left|z_{j, l, \mu}\right| .
\end{aligned}
$$

Furthermore, by (3.6) and again the first inequality in (3.11), we obtain

$$
\ln \left|\widetilde{q}_{\Lambda}\left(z_{j, l, \mu}, z_{j, l, \mu}, \widetilde{\alpha} / p_{0}, 3 \delta_{j}\right)\right| \geq\left(S_{\Lambda}^{2}\left(\widetilde{\alpha} / p_{0}\right)-\varepsilon / 4\right)\left|z_{j, l, \mu}\right| .
$$

Let $\left|z_{j, l, \mu}-\lambda_{k}\right| \geq 3 \delta_{j}\left|z_{j, l, \mu}\right|$. Then

$$
\left|z-\lambda_{k}\right| \geq\left|z_{j, l, \mu}-\lambda_{k}\right|-\left|z-z_{j, l, \mu}\right| \geq\left|z_{j, l, \mu}-\lambda_{k}\right|-2 \delta_{j}\left|z_{j, l, \mu}\right| \geq 3^{-1}\left|z_{j, l, \mu}-\lambda_{k}\right| .
$$

By (3.14) and (3.11), it follows that

$$
\begin{aligned}
\ln \left|\widetilde{q}_{\Lambda}\left(z, z_{j, l, \mu}, \tilde{\alpha} / p_{0}, 3 \delta_{j}\right)\right| & \geq \ln \left|\widetilde{q}_{\Lambda}\left(z_{j, l, \mu}, z_{j, l, \mu}, \widetilde{\alpha} / p_{0}, 3 \delta_{j}\right)\right|-M_{\Lambda}\left(z_{j, l, \mu}, \widetilde{\alpha} / p_{0}\right) \ln 3 \\
& \geq\left(S_{\Lambda}^{2}\left(\widetilde{\alpha} / p_{0}\right)-M_{\Lambda}(\alpha) \ln 3-\varepsilon / 2\right)\left|z_{j, l, \mu}\right| \\
& \geq\left(S_{\Lambda}^{2}(\alpha)-M_{\Lambda}(\alpha)-\varepsilon / 2\right)\left|z_{j, l, \mu}\right| .
\end{aligned}
$$


Thus, taking (3.12), (3.13), and (3.11) into account, we arrive at

$$
\begin{aligned}
\ln \left|q_{\Lambda}(z, w, \alpha / 6)\right| & \geq\left(M_{\Lambda}^{1}+S_{\Lambda}^{2}(\alpha)-M_{\Lambda}(\alpha) \ln 3-3 \varepsilon / 4\right)\left|z_{j, l, \mu}\right| \\
& \geq\left(M_{\Lambda}^{1}+S_{\Lambda}^{2}(\alpha)-M_{\Lambda}(\alpha) \ln 3-3 \varepsilon / 4\right)\left(1-2 \delta_{j}\right)^{-1}|z| \\
& \geq\left(M_{\Lambda}^{1}+S_{\Lambda}^{2}(\alpha)-M_{\Lambda}(\alpha) \ln 3-\varepsilon\right)|z| .
\end{aligned}
$$

Remarks. 1. Theorem 3.1 shows that $M_{\Lambda}^{1} \geq S_{\Lambda}^{2}(\alpha)$. Therefore, the quantity $S_{\Lambda}^{2}(\alpha)+M_{\Lambda}^{1}$ can be replaced by $2 S_{\Lambda}^{2}(\alpha)$ in the inequality of item 3) in Lemma 3.1.

2. Clearly, the inequality $S_{\Lambda}^{2}(\widetilde{\alpha})>-\infty$ in Lemma 3.1 is fulfilled whenever $S_{\Lambda}^{1}>-\infty$. Yet it may fail if merely $S_{\Lambda}^{2}$ is finite. Consider the corresponding example. We enumerate all pairs of natural numbers in the same way as in Remark 3 to Theorem 3.1. Then $p(s, l) \geq 2 s, s \geq 1$. Choose a sequence $\left\{\xi_{s}\right\} \subset \partial B(0,1)$ with $\left|\xi_{s}-1\right|=1 / s, s \geq 1$. Next, put $\Lambda=\bigcup_{p=2}^{\infty} \Lambda_{p}$ and

$$
\Lambda_{p(s, l)}=\bigcup_{j=s}^{2 s-1}\left\{\left(2^{p(s, l)}\left(1-\exp \left(-2^{j+1}\right)\right)-m h_{j}\right) \xi_{s}, m=0,1, \ldots, 2^{p(s, l)-j}-1\right\},
$$

where $h_{j}=\left(\exp \left(-2^{j}\right)-\exp \left(-2^{j+1}\right)\right) 2^{j}$. We note that the sequence $\Lambda^{\prime}$ composed of the numbers $\left|\lambda_{k}\right|, k \geq 1$ (where the $\lambda_{k}$ are the points of $\Lambda$ ) is a part of the sequence occurring in Remark 2 to Theorem 3.1. Thus, $M_{\Lambda}^{1} \geq-32$. In particular, $M_{\Lambda}=0$. If $\xi \neq 1, \xi_{s}, s \geq 1$, then for some $\alpha>0$ the disk $B(t \xi, \alpha t), t>0$, does not contain any $\lambda_{k}$. Consequently, $S_{\Lambda, \xi}^{1}=0$. Let $\xi=\xi_{s}$. Then there exists $\alpha \in\left(0, \exp \left(-2^{2 s-1}\right)-\exp \left(-2^{2 s}\right)\right)$ such that the disk $B(t \xi, \alpha t), t>0$, may contain only points of the group $\Lambda_{p(s, l)}$, the distances between which are at least $h_{2 s-1}$. Arguing as in case 1) in Remark 3 to Theorem 3.1, it is easy to show that $S_{\Lambda, \xi}^{1}=0$. Finally, we also have $S_{\Lambda, 1}^{1}=0$. Indeed, for sufficiently small $\alpha \in(0,1 / 3)$, the disk $B(t, \alpha t), t>0$, may only contain points of one group $\Lambda_{p(s, l)}$, whose distances to $t$ are at least $t / 2 s$. Furthermore, $1 / 2 s \leq \alpha, t \geq 2^{p(s, l)-1}$, and the number of points in the group $\Lambda_{p(s, l)}$ is at most $2^{p(s, l)-s+1}$. Then

$$
\begin{aligned}
\ln \left|\widetilde{q}_{\Lambda}(t, t, \alpha, \delta)\right| & \geq \ln \left|q_{\Lambda}(t, t, \alpha)\right| \geq-\frac{2^{p(s, l)-s+1}}{t} \ln (8 s \alpha) \\
& \geq-2^{-s+2} \ln (8 s \alpha) \geq-2^{-s+2} \ln (8 s) .
\end{aligned}
$$

This yields the required identity. Thus, $S_{\Lambda}^{2}=0$. At the same time, as in Remark 2 to Theorem 3.1 , it can be shown that $S_{\Lambda, \xi_{s}}^{1}\left(\exp \left(-2^{j}\right)\right) \leq-s, s \geq j$. Consequently, $S_{\Lambda}^{2}(\alpha) \equiv-\infty$.

Lemma 3.2. Let $|\xi|=1$, and let $\Lambda=\left\{\lambda_{k}, n_{k}\right\}$ be such that $S_{\Lambda, \xi}^{1}(\widetilde{\alpha})>-\infty$ for some $\tilde{\alpha} \in(0,1 / 3)$. Then $M_{\Lambda, \xi}^{1}>-\infty$ and there exists an infinitely growing sequence $r_{m}>0$, $m \geq 1$, with the following properties:

1) $\lim _{m \rightarrow \infty} r_{m+1} / r_{m}=1$;

2) for every $\varepsilon>0$ and $\alpha \in(0, \widetilde{\alpha}]$, there exists $R>0$ with

$$
\begin{aligned}
& \ln \left|q_{\Lambda}(z, w, \alpha / 6)\right| \geq\left(S_{\Lambda, \xi}^{1}(\alpha)+M_{\Lambda, \xi}^{1}-M_{\Lambda, \xi}(\alpha) \ln 3-\varepsilon\right)|z|, \\
& z \in\left\{r_{m} \xi\right\}_{m=1}^{\infty} \cap B(w, \alpha|w| / 6), \quad w \in \mathbb{C} \backslash B(0, R) .
\end{aligned}
$$

Proof. We argue as in the preceding lemma. As in that proof, we choose $\delta_{p}, R_{p}$, and $\mu_{p}$ that satisfy (3.8) with $S_{\Lambda}^{2}(\widetilde{\alpha} / s)$ and $M_{\Lambda}^{1}$ replaced by $S_{\Lambda, \xi}^{1}(\widetilde{\alpha} / s)$ and $M_{\Lambda, \xi}^{1}$, and with the points $t e^{i l \varphi_{p}}$ and $w$ both replaced by $t \xi$. Put $z_{p, \mu}=R_{p}\left(1+\delta_{p}\right)^{\mu} \xi, p \geq 1, \mu=0, \ldots, \mu_{p}$. As in Lemma 3.1, we invoke the Cartan theorem. This will result in formula (3.9) in which the index $l$ is dropped and $M_{\Lambda}^{1}$ is replaced by $M_{\Lambda, \xi}^{1}$. Let $\left\{r_{m}\right\}$ be the sequence of the numbers $R_{p}\left(1+\delta_{p}\right)^{\mu}\left(1+\tau_{p, \mu} \delta_{p}\right), p \geq 1, \mu=0, \ldots, \mu_{p}$, enumerated in the increasing 
order. Since $\delta_{p} \rightarrow 0$, statement 1 ) of the lemma follows. To obtain 2), we argue as in Lemma 3.1 by using (3.9) and (3.6).

A converse to Lemma 3.2 holds in the following sharp form.

Lemma 3.3. Let $|\xi|=1$, and let $\Lambda=\left\{\lambda_{k}, n_{k}\right\}$. Suppose that, for some $\widetilde{\alpha} \in(0,1 / 3)$, $a>0$, and a sequence $\left\{w_{m}\right\}$ with unboundedly growing moduli and with $w_{m} /\left|w_{m}\right| \rightarrow \xi$, $\left|w_{m+1}\right| /\left|w_{m}\right| \rightarrow 1$ as $m \rightarrow \infty$, we have

$$
\ln \left|q_{\Lambda}\left(w_{m}, w_{m}, \widetilde{\alpha}\right)\right| \geq-a\left|w_{m}\right|, \quad m \geq 1 .
$$

Then for every $\varepsilon>0, \alpha \in(0, \widetilde{a}], \delta \in(0, \alpha)$, and an arbitrary sequence $\left\{z_{p}\right\}$ such that $\left|z_{p}\right| \rightarrow \infty$ and $z_{p} /\left|z_{p}\right| \rightarrow \xi$ as $p \rightarrow \infty$, there exists an index $p_{0}$ with

$$
\ln \left|\widetilde{q}_{\Lambda}\left(z_{p}, z_{p}, \alpha, \beta\right)\right| \geq-(a+\varepsilon)\left|z_{p}\right|, \quad p \geq p_{0} .
$$

In particular, $S_{\Lambda, \xi}^{1}(\alpha) \geq-a$.

Proof. Fix $\varepsilon>0, \alpha \in(0, \tilde{\alpha} / 2]$, and $\delta \in(0, \alpha)$. By assumption and the definition of $q_{\Lambda}$, we obtain

$$
-a\left|w_{m}\right| \leq \ln \left|q_{\Lambda}\left(w_{m}, w_{m}, \widetilde{\alpha}\right)\right| \leq-M_{\Lambda}\left(w_{m}, \widetilde{\alpha}\right) \ln 3, \quad m \geq 1 .
$$

Therefore, for some $\gamma \in(1 / 2,1)$ we have $M_{\Lambda}\left(w_{m}, 2 \alpha\right) \ln \gamma \geq-\varepsilon\left|w_{m}\right|, m \geq 1$. Put $\beta=(1-\gamma) / \gamma$. We choose $m_{0}$ such that $\left|w_{m} /\right| w_{m}|-\varepsilon|<\beta \delta / 3,|| w_{m+1}|/| w_{m}|-1|<\beta \delta / 3$ for $m \geq m_{0}$. Let $\left\{z_{p}\right\}$ be a sequence with $z_{p} \rightarrow \infty$ and $z_{p} /\left|z_{p}\right| \rightarrow \xi$ as $p \rightarrow \infty$. We choose $p_{0}$ in such a way that $\left|z_{p} /\right| z_{p}|-\varepsilon|<\beta \delta / 3$ and $\left|z_{p}\right| \geq\left|w_{m_{0}}\right|$ for $p \geq p_{0}$. Fixing $p \geq p_{0}$, we choose $m \geq m_{0}$ with $\left|w_{m}\right| \leq\left|z_{p}\right|<\left|w_{m+1}\right|$. Then $w_{m} \in B\left(z_{p}, \beta \delta\left|z_{p}\right|\right)$ and $B\left(z_{p}, \alpha\left|z_{p}\right|\right) \subset B\left(w_{m}, \widetilde{\alpha}\left|w_{m}\right|\right)$. The last inclusion and the properties of the factors forming $q_{\Lambda}$ (see (2.1)) show that

$$
\ln \left|\widetilde{q}_{\Lambda}\left(w_{m}, z_{p}, \alpha, \beta\right)\right| \geq \ln \left|q_{\Lambda}\left(w_{m}, z_{p}, \alpha\right)\right| \geq \ln \left|q_{\Lambda}\left(w_{m}, w_{m}, \widetilde{\alpha}\right)\right| \geq-a\left|w_{m}\right| \geq-a\left|z_{p}\right| .
$$

Let $\left|\lambda_{k}-z_{p}\right| \geq \delta\left|z_{p}\right|$. Since $w_{m} \in B\left(z_{p}, \beta \delta\left|z_{p}\right|\right)$, we have $\left|\lambda_{k}-z_{p}\right|>\gamma\left|\lambda_{k}-2_{m}\right|$. Therefore,

$$
\begin{aligned}
\ln \left|\widetilde{q}_{\Lambda}\left(z_{p}, z_{p}, \alpha, \delta\right)\right| & \geq \ln \left|\widetilde{q}_{\Lambda}\left(w_{m}, z_{p}, \alpha, \delta\right)\right|+M_{\Lambda}\left(w_{m}, 2 \alpha\right) \ln \gamma \\
& \geq-a\left|z_{p}\right|-\varepsilon\left|w_{m}\right| \geq-(a+\varepsilon)\left|z_{p}\right| .
\end{aligned}
$$

In particular, it follows that $S_{\Lambda, \xi}^{1}(\alpha) \geq-a$.

Theorem 3.2. Let $\Lambda=\left\{\lambda_{k}, n_{k}\right\}$. If $U=\left\{U_{m}\right\}$ is a splitting of $\Lambda$ into relatively small groups, then $S_{\Lambda}^{1} \geq 2 \mathcal{S}_{\Lambda}(U)$. Conversely, there exists a splitting $U$ with $\mathcal{N}(\Lambda, U)=$ $\mathcal{D}(\Lambda, U)=0$ and $\mathcal{S}_{\Lambda}(U) \geq S_{\Lambda}^{2}(\alpha)-M_{\Lambda}(\alpha) \ln 3, \alpha \in(0,1 / 3)$.

Proof. We prove the first claim. If $\mathcal{S}_{\Lambda}(U)=-\infty$, the claim is trivial. Let $\mathcal{S}_{\Lambda}(U)>-\infty$. Fix $\varepsilon>0$. Lemma 2.3 implies that for some family $B_{m}, m \geq 1$, of mutually disjoint open sets we have the following:

$U_{m}=\left\{\lambda_{m, p}\right\} \subset B_{m}$, the diameters $d_{m}$ of $B_{m}$ satisfy

$$
\limsup _{m \rightarrow \infty} \max _{1 \leq p \leq M_{m}} d_{m} /\left|\lambda_{m, p}\right| \leq 2(\mathcal{N}(\Lambda, U)+\mathcal{D}(\Lambda, U))=0
$$

and there exist $R>0$ and $\widetilde{\delta} \in(0,1 / 3)$ with

(3.16) $\ln \left|q_{\Lambda}(z, w, \widetilde{\delta})\right| \geq\left(2 \mathcal{S}_{\Lambda}(U)-\varepsilon\right)|z|, \quad z \in \partial B_{m} \cap B(w, \widetilde{\delta}|w|), \quad|w| \geq R, \quad m \geq 1$.

Let $\alpha \in(0, \tilde{\delta} / 5)$, and let $\delta \in(0, \alpha)$. By (3.15), there is an index $m_{0}$ such that

$$
\sup _{w \in B_{m}} d_{m} /|w|<\delta, \quad m \geq m_{0}
$$

We choose $R_{1} \geq R$ such that every disk $B(w, \alpha|w|), w \in \mathbb{C} \backslash B\left(0, R_{1}\right)$, may only intersect $B_{m}$ with $m \geq m_{0}$. Take $w \in \mathbb{C} \backslash B\left(0, R_{1}\right)$. By (2.1) and (3.16), we have

$$
\ln \left|q_{\Lambda}(z, w, \alpha)\right| \geq\left(2 \mathcal{S}_{\Lambda}(U)-\varepsilon\right)|z|, \quad z \in \partial B_{m} \cap B(w, 5 \alpha|w|), \quad m \geq 1 .
$$


Furthermore, by the definition of $q_{\Lambda}$ we also have

$$
\ln \left|q_{\Lambda}(z, w, \alpha)\right| \geq 0, \quad z \in \partial B(w, 5 \alpha|w|),
$$

Thus, recalling the properties of the factors that form $q_{\Lambda}$, we obtain

$$
\ln \left|\widetilde{q}_{\Lambda}(z, w, \alpha, \delta)\right| \geq \ln \left|q_{\Lambda}(z, w, \alpha)\right| \geq\left(2 \mathcal{S}_{\Lambda}(U)-\varepsilon\right)|w|(1+5 \alpha),
$$

where

$$
z \in\left(\partial B_{m} \cap B(w, 5 \alpha|w|)\right) \cup \partial B(w, 5 \alpha|w|), \quad m \geq 1 .
$$

Since $\widetilde{q}_{\Lambda}(z, w, \alpha, \delta)$ has no zeros on $B(w, 5 \alpha|w|) \backslash \bigcup B_{w}$, inequality (3.18) extends to this set. If $w$ belongs to it, then

$$
\ln \left|\widetilde{q}_{\Lambda}(w, w, \alpha, \delta)\right| \geq\left(2 \mathcal{S}_{\Lambda}(U)-\varepsilon\right)|w|(1+5 \alpha) .
$$

Now, suppose $w \in B_{m}$. By (3.17), we have $B_{m} \subset B(w, \delta|w|)$. Consequently, $\widetilde{q}_{\Lambda}(z, w, \alpha, \delta)$ does not have zeros on $B_{m}$, and again we arrive at (3.19), which implies that $\left.S_{\Lambda}^{1}(\alpha) \geq\left(2 \mathcal{S}_{\Lambda}(U)-\varepsilon\right)(1+5 \alpha)\right)$. Since $\varepsilon$ is arbitrary, we obtain $S_{\Lambda}^{1} \geq 2 \mathcal{S}_{\Lambda}(U)$.

Now, we prove the second claim. If $S_{\Lambda}^{2}(\alpha)=-\infty$, the trivial partition fits. Suppose that $S_{\Lambda}^{2}(\alpha)>-\infty$. Then $M_{\Lambda}^{1} \geq S_{\Lambda}^{2}(\alpha)$ by Theorem 3.1. We apply Theorems 3.1 and 2.4 to obtain a partition $U$ with

$$
\mathcal{S}_{\Lambda}(U) \geq S_{\Lambda}^{2}(\alpha)+M_{\Lambda}^{1}-M_{\Lambda}(\alpha) \ln 3 \geq 2 S_{\Lambda}^{2}(\alpha)-M_{\Lambda}(\alpha) \ln 3
$$

and $\mathcal{D}(\Lambda, U)=0$. It remains to observe that the last identity entails the estimate $\mathcal{N}(\Lambda, U) \leq M_{\Lambda}$, and $M_{\Lambda}=0$ because $M_{\Lambda}^{1}$ is finite.

Remarks. 1. Theorem 3.2 implies that the quantities $S_{\Lambda}^{1}$ and $S_{\Lambda}^{2}(\alpha)$ are finite or infinite simultaneously.

2. Theorem 3.2 shows also that the condition $\mathcal{S}_{\Lambda}=0$ implies $S_{\Lambda}^{1}=0$. The converse is not true. Indeed, let $\Lambda=\left\{\lambda_{k}, 1\right\}$, where $\lambda_{2 k}=2^{k}$ and $\lambda_{2 k+1}=2^{k}+b_{k}, b_{k}>0$, $\ln b_{k} / 2^{k} \rightarrow-\infty$. Then

$$
\mathcal{S}_{\Lambda} \leq \lim _{\delta \rightarrow 0} \liminf _{k \rightarrow \infty} 2^{-k} \ln \left(b_{k} / 3 \delta 2^{k}\right)=\lim _{k \rightarrow \infty} 2^{-k} \ln b_{k}=-\infty .
$$

At the same time, for the splitting in which $U_{m}=\left\{\lambda_{2 m}, \lambda_{2 m+1}\right\}, m \geq 1$, for sufficiently small $\delta$ we have $q_{\Lambda, U}^{m, l}(z, \delta) \equiv 1$. Therefore, $\mathcal{S}_{\Lambda}(U)=0$. But then $S_{\Lambda}^{1}=0$ by Theorem 3.2.

3. In the example in Remark 2 to Theorem 3.1 it was shown that $S_{\Lambda}^{1}=-\infty$. Consequently, by Theorem 3.2, the sequence $\Lambda$ constructed in that example cannot be split into relatively small groups $U=\left\{U_{m}\right\}$ in such a way that $\mathcal{S}_{\Lambda}(U)>-\infty$. The same can be said about the sequence $\Lambda$ in Remark 2 to Lemma 3.1, because $S_{\Lambda}^{2}(\alpha)=-\infty$ for $\alpha \in(0,1 / 3)$ in that situation.

We give yet another example, which illustrates Theorem 3.2 and which will be used in the next section. Let a sequence $\Lambda=\left\{\lambda_{k}, n_{k}\right\}_{k=1}^{\infty}$ be such that

$$
M_{\Lambda}^{2}=\limsup _{\delta \rightarrow 0} \limsup _{|z| \rightarrow \infty} M_{\Lambda}(w, \delta) / \delta|w|<\infty .
$$

This condition is fulfilled if $\Lambda$ has angular density (see [9, Chapter II, §1]) or is a part of a sequence with finite angular density, i.e., if $\Lambda$ is a part of the zero set for a function of completely regular growth (see [9, Chapter III, $\S 3$, Theorem 4]).

Since $M_{\Lambda}^{2}<\infty$, we have $M_{\Lambda}^{0}<\infty$. Then $S_{\Lambda}^{1}=0$ by Theorem 3.1 , and Theorem 3.2 implies the existence of a splitting $U$ into relatively small groups with $\mathcal{S}_{\Lambda}(U)=0$. Note that the condition $M_{\Lambda}^{2}<\infty$ may be fulfilled for a sequence $\Lambda$ that is not necessarily a part of the zero set of some function of regular growth. Let $\Lambda=\bigcup \Lambda_{p}$, where each $\Lambda_{p}$ is a certain subset of the circle $|\lambda|=R_{p}$ and $R_{p+1} \geq(1+\widetilde{\delta}) R_{p}, p \geq 1, \widetilde{\delta}>0$. More specifically, $\Lambda_{p}$ is defined as follows. Choose some chord $[z(p), \widetilde{z}(p)]$ of the circle $|\lambda|=R_{p}$ 
that is orthogonal to the radius-vector $\bar{r}_{p}=R_{p} e^{i \varphi_{p}}$, intersects this radius-vector, and contracts an angle $\varphi_{p} \geq \varphi$. Let $h>0$, and let $z_{l}(p), l=1, \ldots, s(p)$, be points of the chord $[z(p), \widetilde{z}(p)]$ dividing this chord into equal segments of length at least $h . \Lambda_{p}$ is defined as the set of points of intersection of the circle $|\lambda|=R_{p}$ and the straight lines parallel to $\bar{r}_{p}$ and passing through $z_{l}(p)$. If $\varphi>0$, then the maximal density of $\Lambda$ is $+\infty$ :

$$
\limsup _{\delta \rightarrow 0} \limsup _{|z| \rightarrow \infty}(N(\Lambda, R)-N(\Lambda,(1-\delta) R)) / \delta R=\limsup _{\delta \rightarrow 0} \limsup _{p \rightarrow \infty} l(p) / \delta R_{p}=+\infty .
$$

Therefore, $\Lambda$ is not a part of a sequence with angular density, i.e., it is not a part of the zero set of a function of regular growth. At the same time, it can easily be observed that $M_{\Lambda}^{2}<\infty$. Then, as above, there exists a splitting $U$ into relatively small groups with $\mathcal{S}_{\Lambda}(U)=0$. We show that the trivial splitting can be taken for $U$. Let $U=\left\{U_{k}\right\}$, where $U_{k}=\left\{\lambda_{k}\right\}$ and $\tilde{l}(p)$ is the maximal of the natural numbers that are strictly smaller than $\delta R_{p} / h$. If $\delta>0$ is sufficiently small, then the disk $B\left(\lambda_{k}, \delta R_{p}\right)$ contains only points of $\Lambda$ (among all points of $\Lambda_{p}$ ). Then for $\delta$ as indicated above we have

$$
\begin{aligned}
\frac{\ln \left|q_{\Lambda, U}^{k}\left(\lambda_{k}, \delta\right)\right|}{\left|\lambda_{k}\right|} & =\sum_{\substack{\lambda_{S} \in B\left(\lambda_{k}, \delta\left|\lambda_{k}\right|\right) \\
S \neq k}} \frac{1}{R_{p}} \ln \frac{\left|\lambda_{k}-\lambda_{S}\right|}{3 \delta\left|\lambda_{S}\right|} \geq \frac{1}{R_{p}} \ln \frac{(\widetilde{l}(p) !)^{2} h^{2 \tilde{l}(p)}}{\left(3 \delta R_{p}\right)^{2 \tilde{l}(p)}} \\
& \geq \frac{2 \tilde{l}(p)}{R_{p}} \ln \frac{h \tilde{l}(p)}{9 \delta R_{p}} \geq \frac{2 \delta}{h} \ln \frac{\delta R_{p}-h}{9 \delta R_{p}} .
\end{aligned}
$$

It follows that $\mathcal{S}_{\Lambda}=\mathcal{S}_{\lambda}(U)=0$.

\section{$\S 4$. Completion of a complex sequence}

In this section we construct a special completion of the sequence $\Lambda$ (split into groups) up to the zero set of an entire function of exponential type (i.e., a function $f$ satisfying $\ln |f(w)| \leq A+B|w|, w \in \mathbb{C}$, with some $A$ and $B)$.

Theorem 4.1. Let a sequence $\Lambda=\left\{\lambda_{k}, n_{k}\right\}_{k=1}^{\infty}$ be split into groups $U=\left\{U_{m}\right\}_{m=1}^{\infty}$ in such a way that $\mathcal{S}_{\Lambda}(U)>-\infty, \mathcal{N}(\Lambda, U), \mathcal{D}(\Lambda, U)<+\infty$. There exists a sequence $\Lambda^{\prime}=\left\{\xi_{l}, 1\right\}_{l=1}^{\infty}$ having no common points with $\Lambda$ and satisfying the following conditions:

1) $\widetilde{\Lambda}=\Lambda \cup \Lambda^{\prime}$ is the zero set (with multiplicities) of an entire function of exponential type;

2) for the splitting $\widetilde{U}=U \cup U^{\prime}$ of the sequence $\widetilde{\Lambda}$, where $U^{\prime}$ is the trivial splitting of $\Lambda^{\prime}$, we have $\mathcal{S}_{\widetilde{\Lambda}}(\widetilde{U})>-\infty, \mathcal{N}(\widetilde{\Lambda}, \widetilde{U})=\mathcal{N}(\Lambda, U), \mathcal{D}(\widetilde{\Lambda}, \widetilde{U})=\mathcal{D}(\Lambda, U)$.

Proof. By Lemma 2.2, the quantity $M_{\Lambda}$ is finite. So, $N(\Lambda)$ is finite by Lemma 2.1. Then Lemma 2.5 implies the existence of $a>0, \delta_{1} \in(0,1 / 3)$, and of numbers $R_{p}>0$ (where $p \geq 1$ ) such that

$$
\begin{aligned}
(1+1 / 30) R_{p} & \leq R_{p+1} \leq(1+1 / 2) R_{p}, \quad p \geq 1, \\
\ln \left|q_{\Lambda}\left(z, w, \delta_{1}\right)\right| & \geq-a|z|, \quad z \in \partial B\left(0, R_{p}\right) \cap B\left(w, \delta_{1}|q|\right), \quad p \geq 1, \quad|w| \geq 2 .
\end{aligned}
$$

The last inequality implies the absence of the $\lambda_{k}$ on the circle $|z|=R_{p}$. Put

$$
A_{p}=\sum_{R_{p}<\left|\lambda_{k}\right|<R_{p+1}} \frac{n_{k}}{\lambda_{k}}=t_{p} e^{-i \varphi_{p}}, \quad p \geq 1 .
$$

Since $N(\Lambda)<\infty$, for some $c>0$ we have $N\left(\Lambda, R_{p}\right) \leq c R_{p}, p \geq 1$. Taking (4.1) into account, we obtain

$$
t_{p} \leq N\left(\Lambda, R_{p+1}\right) / R_{p} \leq c R_{p+1} / R_{p} \leq 3 c / 2, \quad p \geq 1 .
$$


Let $[z(p), \widetilde{z}(p)]$ be the chord of the circle $|\xi|=R_{p}$ that is orthogonal to the radius-vector $\bar{r}_{p}=-R_{p} e^{i \varphi_{p}}$, intersects it at the point $z_{0}(p)$, and contracts an angle of opening $\pi / 3$ (the length of this chord is $\left.R_{p}\right)$. Fix $h \in(0,1 / 3 c)$. We denote by $s(p)$ the maximal natural number not exceeding $R_{p} / 2 h$, and by $z_{l}(p), l=1, \ldots, s(p)-1$, the points of the interval $\left(z(p), z_{0}(p)\right)$ that split it into intervals of length $R_{p} / 2 s(p)$. Let $z_{l}(p), l=$ $-1, \ldots,-s(p)+1$, be the points of the interval $\left(z_{0}(p), \widetilde{z}(p)\right)$ symmetric to the above points with respect to $z_{0}(p)$. Also, we put $z_{s(p)}(p)=z(p)$ and $z_{-s(p)}(p)=\widetilde{z}(p)$. The points of intersection of the straight lines parallel to the vector $\bar{r}_{p}=R_{p}$ and passing through $z_{l}(p)$, $-s(p) \leq l \leq s(p)$, with the circle $|\xi|=R_{p}$, will be denoted by $\xi_{l}(p)$.

We define sets $\Lambda^{\prime}(p), p \geq 2$. If $t_{p-1} R_{p} \leq 1$, we put $\Lambda^{\prime}(p)=\varnothing$. Suppose that $t_{p-1}>1 / R_{p}=1 /\left|\xi_{0}(p)\right|$. Since $1 / \xi_{1}(p)+1 / \xi_{-l}(p)=-\rho_{p, l} e^{-i \varphi_{p}}$, where $\rho_{p, l} \geq 1 / R_{p}$, the choice of $h$ and $s(p)$ together with formula $(4.3)$ for $l(p)=s(p)$ shows that

$$
\left|\sum_{-l(p) \leq l \leq l(p)} \frac{1}{\xi_{l}(p)}\right| \geq \frac{s(p)+1}{R_{p}} \geq \frac{1}{2 h} \geq \frac{3 c}{2} \geq t_{p-1}>\frac{1}{\left|\xi_{0}(p)\right|} .
$$

The quantity on the left in these inequalities decreases when $l(p)$ decreases. Therefore, there exists an index $1 \leq l(p) \leq s(p)$ such that

$$
\left|\sum_{-l(p) \leq l \leq l(p)} \frac{1}{\xi_{l}(p)}\right| \geq t_{p-1} \geq\left|\sum_{-l(p)+1 \leq l \leq l(p)-1} \frac{1}{\xi_{l}(p)}\right| .
$$

Also, we have

$$
\left|A_{p-1}+\sum_{-l(p)+1 \leq l \leq l(p)-1} \frac{1}{\xi_{l}(p)}\right| \leq\left|\frac{1}{\xi_{-l(p)}(p)}+\frac{1}{\xi_{l(p)}(p)}\right| \leq \frac{2}{R_{p}} .
$$

We take the collection $\xi_{l}(p),-l(p)+1 \leq l \leq l(p)-1$, for $\Lambda^{\prime}(p)$. Thus, if $\Lambda^{\prime}(p)=\varnothing$, then

$$
\left|A_{p-1}\right|=t_{p-1} \leq 1 / R_{p} \leq 2 / R_{p}
$$

Otherwise we have (4.4).

Put $\Lambda^{\prime}=\bigcup_{p \geq 2} \Lambda^{\prime}(p)=\left\{\xi_{l}, 1\right\}_{l=1}^{\infty}$. Let $R>R_{2}$ and $j \geq 2$ satisfy the inequalities $R_{j} \leq R<R_{j+1}$. Relations (4.4), (4.5), and (4.1) imply

$$
\begin{aligned}
\left|\sum_{\substack{R_{1}<\left|\lambda_{k}\right| \\
\left|\xi_{l}\right| \leq R}}\left(\frac{n_{k}}{\lambda_{k}}+\frac{1}{\xi_{l}}\right)\right| & \leq \sum_{1 \leq p \leq j}\left|\sum_{\substack{R_{p-1}<\left|\lambda_{k}\right| \\
\left|\xi_{l}\right| \leq R_{p}}}\left(\frac{n_{k}}{\lambda_{k}}+\frac{1}{\xi_{l}}\right)\right|+\left|\sum_{R_{j}<\left|\lambda_{k}\right| \leq R} \frac{n_{k}}{\lambda_{k}}\right| \\
& \leq \sum_{2 \leq p \leq j} \frac{2}{R_{p}}+\sum_{R_{j}<\left|\lambda_{k}\right| \leq R} \frac{n_{k}}{\left|\lambda_{k}\right|} \\
& \leq \sum_{2 \leq p \leq j} \frac{2}{R_{p}}+\frac{N\left(\Lambda, R_{j+1}\right)}{R_{j}} \leq \frac{2}{R_{1}} \sum_{p=0}^{\infty}(1+1 / 30)^{-p}+\frac{3 c}{2} .
\end{aligned}
$$

Next, the choice of $l(p)$ and $s(p)$ implies

$$
\frac{N\left(\Lambda^{\prime}, R\right)}{R}=\sum_{2 \leq p \leq j} \frac{2 l(p)-1}{R} \leq \sum_{2 \leq p \leq j} \frac{2 s(p)}{R_{j}} \leq \sum_{2 \leq p \leq j} \frac{R_{p}}{h R_{j}} \leq \frac{1}{h} \sum_{p=0}^{\infty}(1+1 / 30)^{-p} .
$$

This means that $N\left(\Lambda^{\prime}\right)<\infty$. Therefore, $N\left(\tilde{\Lambda}^{\prime}\right) \leq N(\Lambda)+N\left(\Lambda^{\prime}\right)<\infty$. By (4.6) and the Lindelöf theorem (see, e.g., [1, Chapter I, $\S 3$, Theorem 3.9]), it follows that $\widetilde{\Lambda}$ is the zero set of an entire function of exponential type.

Thus, we have verified statement 1$)$. Now, we prove statement 2). The formulas $\mathcal{N}(\widetilde{\Lambda}, \widetilde{U})=\mathcal{N}(\Lambda, U)$ and $\mathcal{D}(\widetilde{\Lambda}, \widetilde{U})=\mathcal{D}(\Lambda, U)$ are clear because $U^{\prime}$ is trivial. We show 
that $\mathcal{S}_{\widetilde{\Lambda}}(\widetilde{U})>-\infty$. For this, we must obtain appropriate lower estimates for the functions defining $\mathcal{S}_{\widetilde{\Lambda}}(\tilde{U})$ at the points of the groups $U_{m}=\left\{\lambda_{m, \nu}\right\}$ and $U_{l}^{\prime}=\left\{\xi_{l}\right\}$. First, we consider $U_{l}^{\prime}$. In the last example in the previous section we proved that $\mathcal{S}_{\Lambda^{\prime}}=0$. Thus, there exists $\delta_{2} \in\left(0, \delta_{1}\right)$ and an index $l_{0}$ such that

$$
\ln \left|q_{\Lambda^{\prime}, U^{\prime}}^{l}\left(\xi_{l}, \delta_{2}\right)\right|=\sum_{\substack{\xi_{i} \in B\left(\xi_{l}, \delta_{2}\left|\xi_{l}\right|\right) \\ i \neq l}} \ln \left|\frac{\xi_{i}-\xi_{l}}{3 \delta_{2} \xi_{i}}\right| \geq-\left|\xi_{l}\right|, \quad l \geq l_{0}
$$

Taking (4.2), (2.2), and (2.1) into account, we deduce that

$$
\begin{aligned}
\ln \left|q_{\widetilde{\Lambda}, \widetilde{U}}^{l}\left(\xi_{l}, \delta\right)\right| & \geq \ln \left|q_{\widetilde{\Lambda}, \widetilde{U}}^{l}\left(\xi_{l}, \delta_{2}\right)\right| \\
& =\ln \left|q_{\Lambda^{\prime}, U^{\prime}}^{l}\left(\xi_{l}, \delta_{2}\right)\right|+\ln \left|q_{\Lambda}\left(\xi_{l}, \xi_{l} \delta_{2}\right)\right| \geq-(a+1)\left|\xi_{l}\right|,
\end{aligned}
$$

where $l \geq l_{0},\left|\xi_{l}\right| \geq 2$, and $\delta \in\left(0, \delta_{2}\right)$. Now, we consider the group $U_{m}$. Since $\mathcal{S}_{\Lambda}(U)>$ $-\infty$, there exists $\delta_{3} \in\left(0, \delta_{2}\right), b>0$, and an index $m_{0}$ with

$$
\ln \left|q_{\Lambda, U}^{m, l}\left(\lambda_{m, l}, \delta_{3}\right)\right| \geq-b\left|\lambda_{m, l}\right|, \quad m \geq m_{0}, \quad 1 \leq l \leq M_{m}
$$

Let $\delta \in\left(0, \delta_{3}\right)$, and let $m \geq m_{0}$. If the circle $B\left(\lambda_{m, l}, \delta\left|\lambda_{m, l}\right|\right)$ does not contain any $\xi_{l}$, then by $(2.2)$ we obtain

$$
\ln \left|q_{\widetilde{\Lambda}, \widetilde{U}}^{m, l}\left(\lambda_{m, l}, \delta\right)\right| \geq \ln \left|q_{\widetilde{\Lambda}, \widetilde{U}}^{m, l}\left(\lambda_{m, l}, \delta_{3}\right)\right|=\ln \left|q_{\Lambda, U}^{m, l}\left(\lambda_{m, l}, \delta_{3}\right)\right| \geq-b\left|\lambda_{m, l}\right| .
$$

In the opposite case, by (4.1), for $\delta_{3}$ sufficiently small, the disk $B\left(\lambda_{m, l}, \delta\left|\lambda_{m, l}\right|\right)$ contains points of only one collection $\Lambda^{\prime}(p)$. Let $\xi_{l}(p) \in B\left(\lambda_{m, l}, \delta\left|\lambda_{m, l}\right|\right)$. By (4.2) and (4.1), we obtain

$$
\ln \left|\frac{\xi_{l}(p)-\lambda_{m, l}}{3 \delta\left|\lambda_{m, l}\right|}\right| \geq \ln \left|q_{\Lambda}\left(\xi_{l}(p), \lambda_{m, l}, \delta\right)\right| \geq \ln \left|q_{\Lambda}\left(\xi_{l}(p), \lambda_{m, l}, \delta_{1}\right)\right| \geq-a\left|\xi_{l}(p)\right| .
$$

It follows that

$$
\ln \left|\frac{\xi_{l}(p)-\lambda_{m, l}}{3 \delta\left|\lambda_{l}(p)\right|}\right| \geq \ln \left|\frac{\xi_{l}(p)-\lambda_{m, l}}{3 \delta(1+\delta)\left|\lambda_{m, l}\right|}\right| \geq-\ln (1+\delta)-a(1+\delta)\left|\lambda_{m, l}\right| .
$$

By construction, except for at most two points $\xi_{l}(p)$, we have $\left|\xi_{l}(p)-\lambda_{m, l}\right| \geq i h$, where $i$ is a positive integer depending on $l$. Let $i(p)$ be the maximal positive integer strictly smaller than $\delta\left|\lambda_{m, l}\right| / h$. Then

$$
\begin{aligned}
\ln \left|q_{\Lambda^{\prime}}\left(\lambda_{m, l}, \lambda_{m, l}, \delta\right)\right| & \geq-2 \ln (1+\delta)-2 a(1+\delta)\left|\lambda_{m, l}\right|+\ln \frac{(i(p) !)^{2} h^{2 i(p)}}{\left(3 \delta(1+\delta)\left|\lambda_{m, l}\right|\right)^{2 i(p)}} \\
& \geq-2 \ln (1+\delta)-2 a(1+\delta)\left|\lambda_{m, l}\right|+2 i(p) \ln \left(h i(p) / 9 \delta\left|\lambda_{m, l}\right|\right) \\
& \geq-2 \ln (1+\delta)-2 a(1+\delta)\left|\lambda_{m, l}\right|+\frac{2 \delta\left|\lambda_{m, l}\right|}{h} \ln \frac{\delta\left|\lambda_{m, l}\right|-h}{9 \delta\left|\lambda_{m, l}\right|}
\end{aligned}
$$

Invoking (4.8) and (2.2), we obtain

$$
\begin{aligned}
\ln \left|q_{\widetilde{\Lambda}, \widetilde{U}}^{m, l}\left(\lambda_{m, l} \delta\right)\right| & =\ln \left|q_{\Lambda, U}^{m, l}\left(\lambda_{m, l}, \delta\right)\right|+\ln \left|q_{\Lambda^{\prime}}\left(\lambda_{m, l}, \lambda_{m, l}, \delta\right)\right| \\
& \geq-b\left|\lambda_{m, l}\right|-2 \ln (1+\delta)-2 a(1+\delta)\left|\lambda_{m, l}\right|+\frac{2 \delta\left|\lambda_{m, l}\right|}{h} \ln \frac{\delta\left|\lambda_{m, l}\right|-h}{9 \delta\left|\lambda_{m, l}\right|} .
\end{aligned}
$$

By (4.7) and (4.9), it follows that $\mathcal{S}_{\widetilde{\Lambda}}(\widetilde{U})>-b-2 a$. This finishes the proof of the theorem.

Remarks. 1. It can be seen from the proof that the completion of $\Lambda$ does depend on $\Lambda$ but is independent of a splitting $U$. 
2. If $U$ is a splitting into relatively small groups, then $\widetilde{U}$ is also a splitting into relatively small groups. Moreover, if $\mathcal{S}_{\Lambda}>-\infty$, then $\mathcal{S}$ is also finite.

\section{§5. LOWER ESTIMATES FOR AN ENTIRE FUNCTION OF EXPONENTIAL TYPE}

In this section, we establish a relationship between lower estimates for an entire function of exponential type and splittings of its zero set into groups.

Theorem 5.1. Let $f$ be an entire function of exponential type, and $\Lambda=\left\{\lambda_{k}, n_{k}\right\}$ its (multiple) zero set. Suppose we are given a splitting $U=\left\{U_{m}\right\}, U_{m}=\left\{\lambda_{m, l}\right\}_{l=1}^{M_{m}}$ of $\Lambda$ with $\mathcal{S}_{\Lambda}(U)>-\infty$ and $\mathcal{N}(\Lambda, U), \mathcal{D}(\Lambda, U)<+\infty$. Then there exist positive numbers $\left\{\gamma_{m, l}\right\}_{l=1, m=1}^{M_{m}, \infty}$ such that

1)

$$
\begin{gathered}
\liminf _{m \rightarrow \infty} \min _{1 \leq l, j \leq M_{m}} \ln \gamma_{m, l} /\left|\lambda_{m, j}\right| \geq(1+\mathcal{D}(\Lambda, U)) \mathcal{S}_{\Lambda}(U) \\
\limsup _{m \rightarrow \infty} \max _{1 \leq l, j \leq M_{m}} \gamma_{m, l} /\left|\lambda_{m, j}\right| \leq \mathcal{N}(\Lambda, U)+\mathcal{D}(\Lambda, U)
\end{gathered}
$$

2) the sets $B_{m}=\bigcup_{l=1}^{M_{m}} B\left(\lambda_{m, l}, \gamma_{m, l}\right), m=1,2, \ldots$, are mutually disjoint;

3) the diameters $d_{m}$ of the sets $B_{m}$ satisfy the relation

$$
\limsup _{m \rightarrow \infty} \max _{1 \leq p \leq M_{m}} d_{m} /\left|\lambda_{m, p}\right| \leq 2(\mathcal{N}(\Lambda, U)+\mathcal{D}(\Lambda, U)) ;
$$

4) for every $\theta \in(0,1)$ there exist $b, b_{1}>0$ such that $\ln |f(z)| \geq-b_{1}-b|z|$, $z \in$ $\left(B_{m} \backslash \widetilde{B}_{m}(\theta)\right), m \geq 1$, where $\widetilde{B}_{m}(\theta)=\bigcup_{l=1}^{M_{m}} B\left(\lambda_{m, l}, \theta \gamma_{m, l}\right)$.

Proof. By Lemma 2.3, there exist $\gamma_{m, l}>0$ such that statements 1)-3) are fulfilled and for every $\theta \in(0,1)$ there exists $R>0$ and $\delta \in(0,1 / 3)$ such that

$$
\begin{aligned}
& \ln \left|q_{\Lambda}(z, w, \delta)\right| \geq\left(2 S_{\Lambda}(U)-1\right)|z|, \\
& z \in\left(B_{m} \backslash \widetilde{B}_{m}(\theta)\right) \cap B(w, \delta|w|), \quad|w| \geq R, \quad m \geq 1 .
\end{aligned}
$$

We prove statement 4$)$. By (2.1), we may assume that $\delta<1 / 5$. Since $f$ is an entire function of exponential type, for some $A>0$ we have

$$
\ln |f(\lambda)| \leq A|\lambda|, \quad|\lambda| \geq(1-5 \delta) R .
$$

By a theorem on a lower estimate on circles for an entire function of exponential type (see, e.g., [1, Chapter I, §4, Theorem 4.3]), there exists $a>0$ and an unboundedly growing sequence $\left\{R_{p}\right\}$ of positive numbers such that $R_{p+1} \leq(1+\delta / 2) R_{p}$ and

$$
\ln |f(\lambda)| \geq-a|\lambda|, \quad|\lambda|=R_{p}, \quad p \geq 1 .
$$

Let $z \in\left(B_{m} \backslash \widetilde{B}_{m}\right)$ satisfy $|z| \geq \max \left\{R, R_{1}\right\}$. We choose $p$ with $R_{p}<|z| \leq R_{p+1}$. Then $z$ belongs to $B(w, \delta|w| / 2)$ for some $w \in \partial B\left(0, R_{p+1}\right)$. Consider the function $h(\lambda)=$ $f(\lambda)\left(q_{\Lambda}(\lambda, w, \delta)\right)^{-1}$. This function is entire and has no zeros in that disk $B(w, \delta|w|)$. Since $\left|q_{\Lambda}(\lambda, w, \delta)\right| \leq 1$ in this disk, we have $|h(w)| \geq|f(w)|$. The definition of $q_{\Lambda}$ shows that $\left|q_{\Lambda}(\lambda, w, \delta)\right| \geq 1, \lambda \in \partial B(w, 5 \delta|w|)$. Taking (5.3) and (5.2) into account, we see that

$$
\begin{aligned}
\ln \left|h(\lambda) h^{-1}(w)\right| & \leq \ln |h(\lambda)|+a|w| \leq \ln |f(\lambda)|+a|w| \leq A|\lambda|+a|w| \\
& \leq(A(1+5 \delta)+a)|w|, \quad \lambda \in \partial B(w, 5 \delta|w|) .
\end{aligned}
$$

A lower estimate for an analytic function without zeros (see, e.e., [1, Chapter I, §4, Lemma 4.3]) implies

$$
\ln \left|h(\lambda) h^{-1}(w)\right| \geq-2(A(1+5 \delta)+a)|w|, \quad \lambda \in B(w, \delta|w| / 2) .
$$


By (5.3) and (5.1), it follows that

$$
\begin{aligned}
\ln |f(z)| & =\ln |h(z)|+\ln \left|q_{\Lambda}(z, w, \delta)\right| \\
& \geq-2(A(1+5 \delta)+a)|w|+\ln |h(w)|+\left(2 \mathcal{S}_{\Lambda}(U)-1\right)|z| \\
& \geq-(2 A(1+5 \delta)+3 a)|w|+\left(2 \mathcal{S}_{\Lambda}(U)-1\right)|z| \geq-b|z|,
\end{aligned}
$$

where $b=(2 A(1+5 \delta)+3 a)(1-\delta / 2)^{-1}-2 S_{\Lambda}(U)+1$. Choosing an appropriate $b_{1}>0$, we obtain 4$)$. This proves the theorem.

A converse to Theorem 5.1 also holds in the following form.

Theorem 5.2. Let $f$ be an entire function of exponential type, and let $\Lambda=\left\{\lambda_{k}, n_{k}\right\}$ be its multiple set of zeros. Suppose we are given a sequence of open sets $B_{m}, m \geq 1$, with the following properties:

1) the sets $B_{m}, m \geq 1$, are mutually disjoint, their union contains all $\lambda_{k}$, and each $B_{m}$ contains at least one $\lambda_{k}$;

2) the diameters $d_{m}$ of the sets $B_{m}, m=1,2, \ldots$, satisfy the relation

$$
\limsup _{m \rightarrow \infty} \sup _{\lambda \in B_{m}} d_{m} /|\lambda|=d<\infty
$$

3) there exist $b, R>0$ with $\ln |f(z)| \geq-b|z|, z \in \partial B_{m} \backslash B(0, R), m \geq 1$.

Then the splitting $U=\left\{U_{m}\right\}$ of $\Lambda$ such that $U_{m}$ consists of all $\lambda_{k}$ belonging to $B_{m}$ satisfies the conditions $\mathcal{S}_{\Lambda}(U)>-\infty$ and $\mathcal{D}(\Lambda, U) \leq d$.

Proof. Choose $A>0$ such that $\ln |f(z)| \leq A|z|,|z| \geq R$. Let $\delta \in(0,1 / 3)$, and let $w \in \mathbb{C} \backslash B(0,(1+\delta) R)$. Since $\left|q_{\Lambda}(z, w, \delta)\right| \geq 1$ and $z \in \partial B(w, 5 \delta|w|)$, we have

$$
\ln |f(z)|-\ln \left|q_{\Lambda}(z, w, \delta)\right| \leq \ln |f(z)| \leq A|z| \leq A(1+5 \delta)|w|, \quad z \in \partial B(w, 5 \delta|w|) .
$$

By the maximum principle, the last inequality extends to the disk $B(w, 5 \delta|w|)$. By item 3 ) in the theorem, it follows that

$$
\ln \left|q_{\Lambda}(z, w, \delta) \geq-\left(A(1+5 \delta)(1-\delta)^{-1}+b\right)\right| z \mid, \quad z \in \partial B_{m} \cap B(w, \delta|w|), \quad m \geq 1 .
$$

Application of Lemma 2.4 finishes the proof.

\section{$\S 6$. LOWER INDICATOR}

Let $f$ be an entire function of exponential type. Its lower indicator (see [10, Chapter 4, the Introduction]) is defined to be the function

$$
\underline{h}_{f}(\lambda)=\lim _{\delta \rightarrow 0} \liminf _{t \rightarrow \infty} \frac{1}{\pi \delta^{2}} \int_{B(\lambda, \delta)} \frac{\ln |f(t z)|}{t} d x d y, \quad z=x+i y .
$$

This function is positive homogeneous of degree one, i.e., $\underline{h}_{f}(t \lambda)=t \underline{h}_{f}, t \geq 0$. We signalize that there is a different equivalent definition of the lower indicator, see, e.g., [11].

We present some conditions ensuring the finiteness of the lower indicator. But before we formulate two auxiliary statements, which were proved in a more general form in [12, Proposition 9.3] and [13, Lemma 2.7].

Lemma 6.1. Let $f$ be an entire function of exponential type, let $|\xi|=1$, and let $a \in \mathbb{R}$. If $\underline{h}_{f}(\xi)<a$, then there exists $\delta>0$ and an unboundedly growing sequence $r_{j}>0$ such that $\ln \left|f\left(r_{j} \lambda\right)\right| \leq a r_{j}, \lambda \in B(\xi, \delta), j \geq 1$.

Lemma 6.2. Let $f$ be an entire function of exponential type, let $|\xi|=1$, and let $a \in \mathbb{R}$. If $\underline{h}_{f}(\xi) \geq a$, then there exists a sequence $\left\{\xi_{m}\right\}$ with unboundedly growing moduli and such that $\xi_{m} /\left|\xi_{m}\right| \rightarrow \xi,\left|\xi_{m+1}\right| /\left|\xi_{m}\right| \rightarrow 1$ as $m \rightarrow \infty$ and $\liminf _{m \rightarrow \infty} \ln \left|f\left(\xi_{m}\right)\right| /\left|\xi_{m}\right| \geq a$. 
Remark. Lemma 6.1 implies the converse to Lemma 6.2. Indeed, let $\left\{\xi_{m}\right\}$ be as in Lemma 6.2. Suppose that $\underline{h}_{f}(\xi)<a-2 \varepsilon$ for some $\varepsilon>0$. By Lemma 6.1, we have

$$
\ln |f(\lambda)| \leq(a-\varepsilon) r_{j} \leq(a-2 \varepsilon)(1 \pm \widetilde{\delta})^{-1}|\lambda|, \quad \lambda \in B\left(r_{j} \xi, \widetilde{\delta} r_{j}\right), \quad j \geq 1, \quad \widetilde{\delta} \in(0, \delta)
$$

(the sign in parentheses is opposite to the sign of $a-2 \varepsilon$ ). We choose $\widetilde{\varepsilon} \in(0, \delta)$ in such a way that $(a-2 \varepsilon)(1 \pm \widetilde{\delta})^{-1} \leq a-\varepsilon$. Then, by the last property of the sequence $\left\{\xi_{m}\right\}$, there exists an index $p_{0}$ such that for all $p \geq p_{0}$ the point $\xi_{m}$ does not belong to any disk $B\left(r_{j}, \xi, \widetilde{\delta} r_{j}\right), j \geq 1$. Clearly, this contradicts the properties of $\left\{\xi_{m}\right\}$. Therefore, $\underline{h}_{j}(\xi) \geq a$.

Theorem 6.1. Let $f$ be an entire function of exponential type, $\Lambda=\left\{\lambda_{k}, n_{k}\right\}$ its multiple set of zeros, and $\Gamma$ a cone with vertex at zero. The following statements are equivalent:

1) there exists $\alpha \in(0,1 / 3)$ with $S_{\Lambda, \Gamma}^{2}(\alpha)>-\infty$;

2) there exists $b>0$ with $\underline{h}_{f}(\lambda) \geq-b|\lambda|, \lambda \in \Gamma$.

Proof. 1) $\Longrightarrow 2$ ). We argue in accordance with the pattern of Theorem 5.1. Let $\xi \in$ $\Gamma \cap B(0,1)$. By Lemma 3.2 combined with Theorem 3.1 and Lemma 2.1, we find $R>0$ and an unboundedly growing sequence $r_{m}>0, m \geq 1$, such that $r_{m+1} / r_{m} \rightarrow 1$ as $m \rightarrow \infty$ and

(6.1) $\ln \left|q_{\Lambda}(z, w, \alpha / 6)\right| \geq\left(2 S_{\Lambda, \Gamma}^{2}(\alpha)-(1+\alpha) N(\Lambda) \ln 3-1\right)|z|, \quad z \in\left\{r_{m} \xi\right\} \cap B(w, \alpha|w| / 6)$ for all $w \in \mathbb{C} \backslash R(0, R)$.

As in Theorem 5.1, we have

$$
\begin{aligned}
& \ln |f(\lambda)| \leq A|\lambda|, \quad|\lambda| \geq(1-5 \alpha / 6) R, \\
& \ln |f(\lambda)| \geq-a|\lambda|, \quad|\lambda|=R_{p}, \quad p \geq 1,
\end{aligned}
$$

where $A, a>0$ and $R_{p+1} \leq(1+\alpha / 12) R_{p}$. Let $r_{m} \geq \max \left\{R, R_{1}\right\}$. We choose an index $p$ such that $R_{p}<r_{m} \leq R_{p+1}$. Then $r_{m} \xi \in B\left(R_{p+1} \xi, \alpha R_{p+1} / 12\right)$. Consider the function $h(\lambda)=f(\lambda)\left(q_{\Lambda}\left(\lambda, R_{p+1} \xi, \alpha / 6\right)\right)^{-1}$. As in Theorem 5.1, we obtain

$$
\ln \left|h\left(r_{m} \xi\right) h^{-1}\left(R_{p+1} \xi\right)\right| \geq-2(A(1+5 \alpha / 6)+a) R_{p+1} .
$$

By (6.3) and (6.1), it follows that

$$
\begin{aligned}
& \ln \left|f\left(\xi_{m}\right)\right|=\ln \left|h\left(\xi_{m}\right)\right|+\ln \left|q_{\Lambda}\left(\xi_{m}, R_{p+1}, \xi, \alpha / 6\right)\right| \\
& \geq-2(A(1+5 \alpha / 6)+a) R_{p+1}+\ln \left|h\left(R_{p+1} \xi\right)\right|+\left(2 S_{\Lambda, \Gamma}^{2}(\alpha)-(1+\alpha) N(\Lambda) \ln 3-1\right)\left|\xi_{m}\right| \\
& \geq-b\left|\xi_{m}\right|,
\end{aligned}
$$

where $\xi_{m}=r_{m} \xi$ and $b+(2 A(1+5 \alpha / 6)+3 a)(1-\alpha / 12)^{-1}-2 S_{\Lambda, \Gamma}^{2}(\alpha)+(1+\alpha) N(\Lambda) \ln 3+1$. By the remark to Lemma 6.2 , we have $\underline{h}_{f}(\xi) \geq-b$. Since the indicator is positive homogeneous, statement 2) follows.

$2) \Longrightarrow 1)$. Let $\xi \in \Gamma \cap \partial B(0,1)$. By Lemma 6.2 , there exists a sequence $\left\{\xi_{m}\right\}$ with unboundedly growing moduli and such that $\xi_{m} /\left|\xi_{m}\right| \rightarrow \xi$ and $\left|\xi_{m+1}\right| /\left|\xi_{m}\right| \rightarrow 1$ as $m \rightarrow \infty$, and

$$
\ln \left|f\left(\xi_{m}\right)\right| \geq-(b+1)\left|\xi_{m}\right|, \quad m \geq 1 .
$$

Fix $\alpha \in(0,1 / 5)$. As before, using the properties of $q_{\Lambda}$ and the maximum principle, we obtain

$$
\ln \left|f(\lambda)\left(q_{\Lambda}(\lambda, w, \alpha)\right)^{-1}\right| \leq B|\lambda|, \quad \lambda \in B(w, 5 \alpha|w|), \quad w \in \mathbb{C} \backslash B(0, R)
$$

with some $B, R>0$. By (6.4), it follows that

$$
\ln \left|q_{\Lambda}\left(\xi_{m}, \xi_{m}, \alpha\right)\right| \geq-(b+B+1)\left|\xi_{m}\right|, \quad\left|\xi_{m}\right| \geq R .
$$


Then $S_{\Lambda, \xi}^{1}(\alpha) \geq-b-B-1$ by Lemma 3.3. Consequently, $S_{\Lambda, \Gamma}^{2}(\alpha)>-\infty$, and the theorem follows.

Corollary 1. Let $f$ be an entire function of exponential type, let $\Lambda=\left\{\lambda_{k}, n_{k}\right\}$ be its multiple set of zeros, and let $|\xi|=1$. The indicator $\underline{h}_{f}$ is finite at $\xi$ if and only if $S_{\Lambda, \xi}^{1}>-\infty$.

Recalling Remark 1 to Theorem 3.2, we obtain another corollary.

Corollary 2. Let $f$ be an entire function of exponential type and $\Lambda=\left\{\lambda_{k}, n_{k}\right\}$ its multiple zero set. The indicator $\underline{h}_{f}$ is bounded from below on the unit circle if and only if $S_{\Lambda}^{1}>-\infty$.

Remark. 1. Consider some examples. Let $\Lambda=\left\{\lambda_{k}, n_{k}\right\}$ have finite upper density, and let $|\xi|=1$. Suppose that $M_{\Lambda, \xi} \neq 0$ or, more generally, $M_{\Lambda, \xi}^{1}=-\infty$. Then, by Theorem 3.1 and Corollary 1, we have $\underline{h}_{f}(\xi)=-\infty$ for every entire function $f$ of exponential type that has a zero of multiplicity at least $n_{k}$ at each $\lambda_{k}$. For example, if $\lim _{\sup _{k \rightarrow \infty}} n_{k} /\left|\lambda_{k}\right| \neq 0$, then there exists $\xi$ with $M_{\Lambda, \xi} \neq 0$ and, therefore, $M_{\Lambda, \xi}^{1}=-\infty$.

Let $\Lambda$ be as in Remark 2 to Theorem 3.1. Then $M_{\Lambda}^{1}=M_{\Lambda, 1}^{1}>-\infty$. Nevertheless, $S_{\Lambda}^{1}=S_{\Lambda, 1}^{1}=-\infty$ and, consequently, $\underline{h}_{f}(1)$ is infinite for any $f$ as indicated.

Let $L(\lambda)$ be the entire function of exponential type defined by (1.3), and let $\widetilde{\Lambda}=$ $\Lambda \cup(-\Lambda)$ be its zero set. Then $S_{\Lambda, \Gamma}^{1}(\alpha) \geq S_{\widetilde{\Lambda}, \Gamma}^{1}(\alpha) \geq 2 S_{\Lambda, \Gamma}^{1}(\alpha)$. In the example in Remark 2 to Lemma 3.1, the function $S_{\Lambda, \xi}^{1}$ is identically zero. Therefore, the indicator $\underline{h}_{L}$ is finite everywhere. However, $S_{\Lambda}^{2}(\alpha) \equiv-\infty$. Consequently, $\underline{h}_{L}$ is not bounded below on the unit circle $\left(\underline{h}_{L}\left(\xi_{s}\right) \rightarrow-\infty, s \rightarrow \infty\right)$.

Assume that $\Lambda$ is the zero set of a function $f$. If $M_{\Lambda}^{0}<\infty$, then, by Theorem 3.1 and Corollary 2, the lower indicator of $f$ is bounded on $\partial B(0,1)$. This happens if $M_{\Lambda}^{2}<\infty$. For example, if $\Lambda$ is a part of the zero set of a function of regular growth, then $\inf _{\xi \in \partial B(0,1)} \underline{h}_{L}(\xi)>-\infty$.

In the example in Remark 3 to Theorem 3.1, the quantity $M_{\Lambda}^{0}$ is infinite. At the same time, $S_{\Lambda}^{1}>-\infty$. Consequently, $\underline{h}_{L}$ is bounded below on $\partial B(0,1)$.

If $\mathcal{S}_{\Lambda}>-\infty$ (or, more generally, $\Lambda$ is split into relatively small groups $U=\left\{U_{m}\right\}$ in such a way that $\left.\mathcal{S}_{\Lambda}(U)>-\infty\right)$, then $\inf _{\xi \in \partial B(0,1)} \underline{h}_{L}(\xi)>-\infty$ by Theorem 3.2 and Corollary 2.

2. We compare Theorem 6.1 with the result of the paper [7], where some finiteness conditions for the lower indicator were obtained. Let $f$ be an entire function of exponential type and $\Lambda=\left\{\lambda_{k}, n_{k}\right\}$ its zero set. In [7] it was proved that $\underline{h}_{f}$ is bounded below on $\partial B(0,1) \cap \Gamma$ if and only if

$$
I_{\Lambda, \Gamma}=\lim _{\delta \rightarrow 0} \limsup _{z \rightarrow \infty, z \in \Gamma} \frac{1}{|z|} \int_{\delta}^{1} \frac{M_{\Lambda}(z, \sigma)}{\sigma} d \sigma<\infty .
$$

Thus, by Theorem 6.1, the quantities $S_{\Lambda, \Gamma}^{2}(\alpha)$ and $I_{\Lambda, \Gamma}(1)$ are finite or infinite simultaneously. We look at $I_{\Lambda, \xi}$ more closely. By Lemma 2.1, for large $|z|$ we have

$$
\frac{1}{|z|} \int_{\alpha}^{1} \frac{M_{\Lambda}(z, \sigma)}{\sigma} d \sigma \leq \int_{\alpha}^{1} \frac{2 N(\Lambda)+1}{\sigma} d \sigma=-(2 N(\Lambda)+1) \ln \alpha .
$$

Theorem 3.1 shows that $M_{\Lambda, \xi}^{1} \geq S_{\Lambda, \Gamma}^{2}(\alpha)$. Consequently,

$$
\limsup _{\delta \rightarrow 0} \limsup _{t \rightarrow \infty} \frac{1}{t} \int_{\alpha}^{1} \frac{M_{\Lambda}(t \xi, \delta)}{\sigma} d \sigma=-\limsup _{\delta \rightarrow 0} M_{\Lambda, \xi}(\delta) \ln \delta=-M_{\Lambda, \xi}^{1} \leq-S_{\Lambda, \Gamma}^{2}(\alpha) .
$$


The last estimates mean that the condition $I_{\Lambda, \xi}<\infty$ is equivalent to the finiteness of the quantity

$$
\tilde{I}_{\Lambda, \xi}(\alpha)=\lim _{\delta \rightarrow 0} \limsup _{t \rightarrow \infty} \frac{1}{t} \int_{\delta}^{\alpha} \frac{M_{\Lambda}(t \xi, \sigma)-M_{\Lambda}(t \xi, \delta)}{\sigma} d \sigma .
$$

Using the Jensen formula (as in Theorem 3.1) and the inequalities $\ln \left|\widetilde{q}_{\Lambda}(z, w, \alpha, \delta)\right| \geq 0$ for $z \in \partial B(w, 5 \alpha|w|)$ and $\ln \left|\widetilde{q}_{\Lambda}(w, w, \alpha, \delta)\right| \leq 0$ for $z \in \partial B(w, \alpha|w|)$, it can easily be shown that

$$
\begin{aligned}
\int_{\delta}^{\alpha} \frac{M_{\Lambda}(z, \sigma)-M_{\Lambda}(z, \beta)}{\sigma|z|} & \leq-\frac{\ln \left|\widetilde{q}_{\Lambda}(z, z, \alpha, \beta)\right|}{|z|} \\
& \leq \frac{M_{\Lambda}(z, \alpha) \ln 5}{|z|}+\int_{\delta}^{\alpha} \frac{M_{\Lambda}(z, \sigma)-M_{\Lambda}(z, \delta)}{\sigma|z|} .
\end{aligned}
$$

Therefore, $\widetilde{I}_{\Lambda, \xi}(\alpha) \leq-S_{\Lambda, \xi}^{1}(\alpha) \leq M_{\Lambda, \xi}(\alpha)+\tilde{I}_{\Lambda, \xi}(\alpha)$. If $M_{\Lambda, \xi}^{1}>-\infty$, then $M_{\Lambda, \xi}=0$. Therefore, the inequalities turn into identities in the limit as $\alpha \rightarrow 0$. Thus, yet another criterion for the boundedness of the indicator $\underline{h}_{f}$ on $\partial B(0,1) \cap \Gamma$ is the condition $\inf _{\xi \in \partial B(0,1) \cap \Gamma} \widetilde{I}_{\Lambda, \xi}(\alpha)<\infty$. Formally, it is weaker than (6.5), in particular, for the reason that (6.5) involves a "uniform" upper limit over the entire cone $\Gamma$.

It should also be noted that, unlike $I_{\Lambda, \Gamma}$, the quantity $S_{\Lambda, \Gamma}^{2}(\alpha)$ is defined in terms of the modulus of a polynomial. This feature is quite convenient technically in various applications.

\section{§7. INTERPOLATION PROBLEM}

In this section, we solve a special interpolation problem in the space of entire functions of exponential type. In the last section, this solution will enable us to show that a certain sequence of exponential polynomials related to this problem forms a basis in $\mathcal{W}(\Lambda)$.

Let $\mathcal{P}$ be the space of entire functions of exponential type endowed with the topology of inductive limit of the Banach spaces

$$
\mathcal{P}_{s}=\left\{f \in H(\mathbb{C}):\|f\|_{s}=\sup _{z \in \mathbb{C}}(|f(z)| \exp (-s|z|))<\infty\right\}, \quad s \geq 1 .
$$

Let the sequence $\Lambda=\left\{\lambda_{k}, n_{k}\right\}$ be split into groups $U=\left\{U_{m}\right\}$, where $U_{m}=\left\{\lambda_{m, j}\right\}_{j=1}^{M_{m}}$, let $\gamma_{m}$ be a contour (a simple closed continuous rectifiable curve) enclosing the points of $U_{m}$, and let

$$
\omega_{m}(\lambda)=\prod_{l=1}^{M_{m}}\left(\lambda-\lambda_{m, l}\right)^{n_{m, l}}, \quad m \geq 1 .
$$

For a function $f$ analytic on $\gamma_{m}$ and inside it, we put

$$
P_{m}(\lambda, f)=\frac{1}{2 \pi i} \int_{\gamma_{m}} \frac{f(\zeta)\left(\omega_{m}(\zeta)-\omega_{m}(\lambda)\right)}{(\zeta-\lambda) \omega_{m}(\zeta)} d \zeta, \quad m \geq 1
$$

Formula (7.1) defines the well-known interpolation polynomial of degree at most $N_{m}-1$ that, together with its derivatives of order up to $n_{m, l}-1$, takes at the $\lambda_{m, l}$ the same values as $f$ and its derivatives, i.e.,

$$
P_{m}^{(n)}\left(\lambda_{m, l}, f\right)=f^{(n)}\left(\lambda_{m, l}\right), \quad l=1,2, \ldots, M_{m}, \quad n=0,1, \ldots, n_{m, l}-1 .
$$

For every $s \geq 1$, we introduce the Banach space of complex sequences

$$
R_{s}(\Lambda, U)=\left\{b=\left\{b_{m, j}\right\}_{m=1, j=1}^{\infty, N_{m}}:\|b\|_{s}=\sup _{m, j}\left(\left|b_{m, j}\right| \exp \left(-s\left|\lambda_{m, 1}\right|\right)\right)<\infty\right\} .
$$

Let $R(\Lambda, U)$ denote the inductive limit of these spaces. Also, let $\mathcal{J}(\Lambda)$ be the subset of $\mathcal{P}$ consisting of all functions that vanish at the $\lambda_{k}$ with multiplicity at least $n_{k}$. On the quotient space $\mathcal{P} / \mathcal{J}(\Lambda)$, we introduce the operator $\mathfrak{C}$ acting by the following rule: 
it takes each equivalence class $[f] \in \mathcal{P} / \mathcal{J}(\Lambda)$ to the sequence $b=\left\{b_{m, j}\right\}$ defined by the formula

$$
b_{m, j}=p_{m, j-1}(f), \quad m \geq 1, \quad j=1, \ldots, N_{m},
$$

where the $p_{m, j}(f)$ are the Fourier coefficients of the expansion of $P_{m}(\lambda, f)$ in the monomials $\left(\lambda-\lambda_{m, 1}\right)^{j} / j$ !, i.e.,

$$
P_{m}(\lambda, f)=\sum_{j=0}^{n_{m}-1} p_{m, j}(f) \frac{\left(\lambda-\lambda_{m, 1}\right)^{j}}{j !} .
$$

Let $\varphi$ be a representative of $[f]$. Then $\varphi-f$ vanishes at the points $\lambda_{m, l}$ together with all its derivatives of order up to $n_{m l}-1$ (inclusive). By (7.2), the polynomial $P_{m}(\lambda, \varphi-f)$ also has this property. Since its degree is at most $N_{m}-1$, we have $P_{m}(\lambda, \varphi-f) \equiv 0$. It follows that $p_{m, j}(\varphi-f)=p_{m, f}(\varphi)-P_{m, j}(f)=0, m \geq 1, j=0, \ldots, N_{m}-1$. Consequently, the operator $\mathfrak{C}$ is well defined. It is injective. Indeed, if $p_{m, j}(\varphi)=p_{m, j}(f)$ for all $m$ and $j$, then $P_{m}(\lambda, \varphi-f) \equiv 0$ by (7.4). In view of (7.2), $\varphi-f$ vanishes at all $\lambda_{m, l}$ with multiplicity at least $n_{m, l}$. This means that $\varphi-f \in \mathcal{J}(\Lambda)$, i.e., $[\varphi]=[f]$.

Lemma 7.1. Let $\Lambda$ be split into groups $U=\left\{U_{m}\right\}$ in such a way that $\mathcal{N}(\Lambda, U)$, $\mathcal{D}(\Lambda, U)<+\infty$, and let $s \geq 1$. There exists $C>0$ and an index $p \geq s$ such that

$$
\left|p_{m, j}(f)\right| \leq C\|f\|_{s} \exp \left(p\left|\lambda_{m, 1}\right|\right), \quad f \in \mathcal{P}_{s}, \quad m \geq 1, \quad j=0,1, \ldots, N_{m}-1 .
$$

In particular, the operator $\mathfrak{C}: \mathcal{P} / \mathcal{J}(\Lambda) \rightarrow R(\Lambda, U)$ is continuous.

Proof. By the definition of $\mathcal{N}(\Lambda, U)$ and $\mathcal{D}(\Lambda, U)$, we can find $\alpha>0$ with

$$
N_{m} \leq \alpha\left(\left|\lambda_{m, 1}\right|+1\right), \quad \max _{1 \leq j \leq M_{m}}\left|\lambda_{m, j}-\lambda_{m, 1}\right|<\alpha\left(\left|\lambda_{m, 1}\right|+1\right), \quad m \geq 1 .
$$

Let $\varphi \in \mathcal{P}_{s}$. By (7.5), we have $U_{m} \subset B\left(\lambda_{m, 1}, \alpha\left(\left|\lambda_{m, 1}\right|+1\right)\right)$. Therefore, replacing the contour $\gamma_{m}$ in (7.1) by the circle $\partial B\left(\lambda_{m, 1}, 3 \alpha\left(\left|\lambda_{m, 1}\right|+1\right)\right)$ and taking (7.5) into account, we obtain

$$
\begin{aligned}
& \max _{\lambda \in B\left(\lambda_{m, 1}, 2 \alpha\left(\left|\lambda_{m, 1}\right|+1\right)\right)}\left|P_{m}(\lambda, f)\right| \\
& \leq 3\|f\|_{s} \max _{\xi \in \partial B\left(\lambda_{m, 1}, 3 \alpha\left(\left|\lambda_{m, 1}\right|+1\right)\right)} \exp (s|\xi|) 2\left(4 \alpha\left(\left|\lambda_{m, 1}\right|+1\right)\right)^{N_{m}} \backslash\left(2 \alpha\left(\left|\lambda_{m, 1}\right|+1\right)\right)^{N_{m}} \\
& \leq 6\|f\|_{s} \exp \left(s\left((1+3 \alpha)\left|\lambda_{m, 1}\right|+3 \alpha\right)+\alpha\left(\left|\lambda_{m, 1}\right|+1\right) \ln 2\right) \leq C\|f\|_{s} \exp \left(p\left|\lambda_{m, 1}\right|\right), \quad m \geq 1,
\end{aligned}
$$

where $C=6 \exp (3 s \alpha+\alpha \ln 2)$ and $p \geq s(1+3 \alpha)+\alpha \ln 2$.

We estimate the coefficients $p_{m, j}(f)$ by using the Cauchy inequality and (7.5). We have

$$
\begin{aligned}
\left|p_{m, j}(f)\right| & \leq \frac{j !}{\left(\alpha\left(\left|\lambda_{m, 1}\right|+1\right)\right)^{j}} C\|f\|_{s} \exp \left(p\left|\lambda_{m, 1}\right|\right) \\
& \leq\left(\frac{j}{\alpha\left(\left|\lambda_{m, 1}\right|+1\right)}\right)^{j} C\|f\|_{s} \exp \left(p\left|\lambda_{m, 1}\right|\right) \\
& \leq C\|f\|_{s} \exp \left(p\left|\lambda_{m, 1}\right|\right), \quad m \geq 1, \quad j=0,1, \ldots, N_{m}-1 .
\end{aligned}
$$

Since $C$ and $p$ are independent of $f \in \mathcal{P}_{s}$, the operator $\mathfrak{C}$ is continuous, and the proof is finished.

Now we show that $\mathfrak{C}$ is a surjection. For this, we must solve the interpolation problem (7.3) for an arbitrary sequence $b \in R(\Lambda, U)$. For the first time, a similar problem arose in [14]. It was also treated in [15] and [8] in other situations. The solution will be achieved in two steps. First, we construct a sequence of polynomials that solve the problem for one $U_{m}$ each. Next, we "glue together" these solutions to obtain the required function $f \in \mathcal{P}$. 
Lemma 7.2. Let $\Lambda$ be split into groups $U=\left\{U_{m}\right\}$ in such a way that $\mathcal{N}(\Lambda, U)<+\infty$, and let $d>0, s \geq 1$. Then there exist constants $C>0$ and $p \geq s$ such that for every $b \in R_{s}(\Lambda, U)$ the polynomials

$$
G_{b, m}(\lambda)=\sum_{j=0}^{N_{m}-1} b_{m, j+1} \frac{\left(\lambda-\lambda_{m, 1}\right)^{j}}{j !}, \quad m \geq 1,
$$

possess the following properties:

1) $p_{m, j-1}\left(G_{b, m}\right)=b_{m, j}, j=1, \ldots, N_{m}$

2) $\left|G_{b, m}(\lambda)\right| \leq C\|b\|_{s} \exp \left(p\left|\lambda_{m, 1}\right|\right), \lambda \in B\left(\lambda_{m, 1}, d\left|\lambda_{m, 1}\right|\right)$.

Remark. If $\lambda_{m, 1}=0$ for some (necessarily unique) index, we agree that $m=1$. In this case $B(0, d)$ plays the role of $B\left(\lambda_{1,1}, d\left|\lambda_{1,1}\right|\right)$.

Proof. Let $b \in R_{s}(\Lambda, U)$. The degrees of the polynomials $P_{m}\left(\lambda, G_{b, m}\right)$ and $G_{b, m}(\lambda)$ do not exceed $N_{m}-1$. By (7.2), these polynomials coincide at the points $\lambda_{m, l}$, together with their derivatives of order up to $n_{m, l}-1$ inclusive. Since the number of points in $U_{m}$ (counted with multiplicities) is $N_{m}$, we have $P_{m}\left(\lambda, G_{b, m}\right) \equiv G_{b, m}(\lambda)$. This yields 1 ). We prove 2). We have

$$
\frac{\ln \left(\left(d\left|\lambda_{m, 1}\right|\right)^{j} / j !\right)}{\left|\lambda_{m, 1}\right|} \leq \frac{\ln \left(3 d\left|\lambda_{m, 1}\right| / j\right)}{\left|\lambda_{m, 1}\right| / j} \leq 3 d \sup _{x>0} \frac{\ln x}{x} \leq 2 d, \quad m \geq 1 .
$$

Consequently, for all $m \geq 1$ and $\lambda \in B\left(\lambda_{m, 1}, d\left|\lambda_{m, 1}\right|\right)$ we obtain

$$
\left|G_{b, m}(\lambda)\right| \leq\|b\|_{s} \exp \left(s\left|\lambda_{m, 1}\right|\right) \sum_{j=0}^{N_{m}-1} \frac{\left(d\left|\lambda_{m, 1}\right|\right)^{j}}{j !} \leq N_{m}\|b\|_{s} \exp \left((s+2 d)\left|\lambda_{m, 1}\right|\right) .
$$

But $\mathcal{N}(\Lambda, U)<+\infty$ by assumption. Therefore, for some $C>0$ we have

$$
N_{m} \leq \exp \left|\lambda_{m, 1}\right| \text {. }
$$

Moreover, we may assume that $\sum_{j=0}^{N_{1}-1} d^{j} / j ! \leq C$. Now, taking $p \geq s+2 d+1$, we arrive at 2).

Lemma 7.3. Suppose that $\Lambda$ is split into groups $U$ in such a way that

$$
\mathcal{N}(\Lambda, U), \mathcal{D}(\Lambda, U)<+\infty, \quad \mathcal{S}_{\Lambda}(U)>-\infty,
$$

and let $s \geq 1$. There exist constants $B>0$ and $l \geq s$ such that for every $b \in R_{s}(\Lambda, U)$ there is $\varphi \in \mathcal{P}$ with the following properties:

1) $p_{m, j-1}(\varphi)=b_{m, j}=1, \ldots, N_{m}, m \geq 1$;

2) $\|\varphi\|_{l} \leq B\|b\|_{s}$.

Proof. Let $b \in R_{s}(\Lambda, U)$. By Theorem 4.1, we may supplement $b$ if necessary by zero terms in order to be able to assume that $\Lambda$ is the multiple zero set of a function $f \in \mathcal{P}_{s}$. By Theorem 5.1, there exist $\gamma_{m, l}>0$ such that statements 1)-3) of that theorem are fulfilled and for every $\theta \in(0,1)$ there exist $b, R>0$ such that 4$)$ is true. By 3$)$, we choose $d>0$ with

$$
B_{m} \subset B\left(\lambda_{m, 1}, d\left|\lambda_{m, 1}\right|\right), \quad m \geq 1 .
$$

Since $\mathcal{N}(\Lambda, U), \mathcal{D}(\Lambda, U)<+\infty$, the definition of the set $B_{m}$ and the second inequality in 1) show that for some $\theta \in(0,1 / 2)$ and $a_{1}, a_{2}>0$ we have

$$
\left|\lambda_{m, 1}\right| \leq a_{2}+a_{1}|\lambda|, \quad \lambda \in \widetilde{B}_{m}(2 \theta), \quad m \geq 1 .
$$


Let $G_{b, m}$ be the polynomials defined in (7.6). We shall seek the function $\varphi$ in the form

$$
\varphi(\lambda)=\sum_{m=1}^{\infty} G_{b, m}(\lambda) \sigma_{m}(\lambda)-h(\lambda) f(\lambda),
$$

where $\sigma_{m}$ is an analog of a "cap" and $h$ is a solution of a special $\bar{\partial}$-problem with estimates.

First, we define $\sigma_{m}$. By item 1) in Theorem 5.1, for some $a>0$ we have $\gamma_{m, l} \geq$ $\exp \left(-a\left|\lambda_{m, 1}\right|\right), l=1, \ldots, M_{m}, m \geq 1$. Therefore, there exist functions $\sigma_{m}, m \geq 1$, with the following properties (see, e.g., [16, Theorem 1.4.1 and formula (1.4.2)]): 1) $\left.\left.\left.\sigma_{m} \in C^{\infty}(\mathbb{C}), 2\right) 0 \leq \sigma_{m}(\lambda) \leq 1, \lambda \in \mathbb{C}, 3\right) \sigma_{m}(\lambda)=1, \lambda \in \widetilde{B}_{m}(\theta), 4\right) \sigma_{m}(\lambda)=0$, $\left.\lambda \notin \widetilde{B}_{m}(2 \theta), 5\right)\left|d \sigma_{m}(\lambda) / d \bar{\lambda}\right| \leq B_{1} \exp \left(a\left|\lambda_{m, 1}\right|\right), \lambda \in \mathbb{C}$, where $B_{1}>0$ is independent of $m$.

Now, we can construct the function $h(\lambda)$. For this, consider the function

$$
\nu(\lambda)=(f(\lambda))^{-1} \sum_{m=1}^{\infty} G_{b, m}(\lambda) \frac{d \sigma_{m}(\lambda)}{d \bar{\lambda}} .
$$

By property 5) for $\sigma_{m}$, using item 2) in Lemma 7.2 and also (7.7) and (7.8), we obtain

$$
\left|G_{b, m}(\lambda) d \sigma_{m}(\lambda) / d \bar{\lambda}\right| \leq B_{2}\|b\|_{2} \exp \left((p+a) a_{1}|\lambda|\right), \quad \lambda \in \widetilde{B}_{m}(2 \theta), \quad m \geq 1,
$$

where $B_{2}=C B_{1} \exp \left((p+a) a_{2}\right)$. Now, properties 3) and 4) for $\sigma_{m}$ and item 2) in Theorem 5.1 show that $\nu(\lambda)$ is nonzero only on the sets $\widetilde{B}_{m}(2 \theta) \backslash \widetilde{B}_{m}(\theta), m \geq 1$, which are mutually disjoint. By the above and item 4) in Theorem 5.1, it follows that

$$
|\nu(\lambda)| \leq B_{2}\|b\|_{s} \exp \left(b_{1}+\left(b+(p+a) a_{1}\right)|\lambda|\right)=B_{3}\|b\|_{s} \exp (\rho|\lambda|), \quad \lambda \in \mathbb{C} .
$$

Consequently,

$$
\int_{\mathbb{C}}|\nu(\lambda)|^{2} \exp (-2(\rho+1)|\lambda|) d \tau \leq\left(B_{3}\|b\|_{2}\right)^{2} \int_{\mathbb{C}} \exp (-2|\lambda|) d \tau=\left(B_{4}\|b\|_{s}\right)^{2},
$$

where $d \tau$ is planar Lebesgue measure. Then (see [17, Chapter 3, $\S 6$, Section 2, Theorem 3.6.2]), in the space of locally square integrable functions on $\mathbb{C}$ there exists an element $h$ that satisfies $d h / d \bar{\lambda}=\nu$ (in the sense of distributions) and admits the estimate

$$
\int_{\mathbb{C}}|h(\lambda)|^{2} \exp \left(-2(\rho+2)|\lambda| d \tau \leq\left(B_{4}\|b\|_{2}\right)^{2} .\right.
$$

Thus, we have defined a function $\varphi$. We show that it has the required properties. The definitions of $\varphi, \nu$, and $h$ show that the distributional derivative of $\varphi$ with respect to $\bar{\lambda}$ is zero on $\mathbb{C}$. Consequently, $\varphi$ is an entire function. Note also that the function $h$ is analytic outside the sets $\widetilde{B}_{m}(2 \theta) \backslash \widetilde{B}_{m}(\theta), m \geq 1$. We estimate $|\varphi|$ from above. Choose $\alpha, \beta>0$ with $|f(\lambda)| \leq \beta \exp (\alpha|\lambda|), \lambda \in \mathbb{C}$. Since the sets $\widetilde{B}_{m}(2 \theta), m \geq 1$, are mutually disjoint, we invoke properties 2) and 4) of $\sigma_{m}$ to obtain

$$
\left|\sum_{m=1}^{\infty} G_{b, m}(\lambda) \sigma_{m}(\lambda)\right| \leq B_{t}\|b\|_{s} \exp \left(\alpha_{1}|\lambda|\right), \quad \lambda \in \mathbb{C},
$$

by analogy with (7.9), where $B_{5}=C \exp \left(p a_{2}\right), \alpha_{1}=p a_{1}$. It follows that

$$
|\varphi(\lambda)| \leq B_{5}\|b\|_{s} \exp \left(\alpha_{1}|\lambda|\right)+|h(\lambda)| \beta \exp (\alpha|\lambda|), \quad \lambda \in C .
$$

Now, the mean value inequality for subharmonic functions implies

$$
|\varphi(\lambda)| \leq \frac{1}{\pi} \int_{B(\lambda, 1)}|\varphi(\xi)| d \tau \leq B_{5}\|b\|_{s} \exp \left(\alpha_{1}(|\lambda|+1)\right)+\frac{\beta}{\pi} \exp (\alpha(|\lambda|+1)) \int_{B(\lambda, 1)}|h(\xi)| d \tau
$$


Using (4.10) and the Cauchy-Bunyakovskiǔ inequality, we arrive at

$\int_{B(\lambda, 1)}|h(\xi)| d \tau \leq B_{4}\|b\|_{s}\left(\int_{B(\lambda, 1)} \exp (2(\rho+2)|\xi|) d \tau\right)^{1 / 2} \leq \sqrt{\pi} B_{4}\|b\|_{s} \exp ((\rho+2)(|\lambda|+1))$.

Thus proves statement 2) of the lemma. Now, we verify statement 1 ). Since $\Lambda$ is the multiple zero set for $f$, and the function $h(\alpha)$ is analytic near $\lambda_{m, \nu}$, whereas $\sigma_{m}(\lambda)=1$, we have

$$
\varphi^{(n)}\left(\lambda_{m, \nu}\right)=G_{b, m}^{(n)}\left(\lambda_{m, \nu}\right), \quad n=0,1, \ldots, n_{m, \nu}-1, \quad m \geq 1 .
$$

By (7.2), the polynomial $P_{m}(\lambda, \varphi)-P_{m}\left(\lambda, G_{b, m}\right)$ has at least $N_{m}$ zeros (counted with multiplicity). Its degree is at most $N_{m}-1$. Thus, it is identically zero, i.e., $P_{m}(\lambda, \varphi)$ and $P_{m}\left(\lambda, G_{b, m}\right)$ coincide. To finish the proof, it remains to apply Lemma 7.2.

Since $\mathfrak{C}$ is an injection, Lemmas 7.1 and 7.3 imply the following statement.

Theorem 7.1. Let $U$ be a splitting of $\Lambda$ such that $\mathcal{N}(\Lambda, U), \mathcal{D}(\Lambda, U)<\infty$, and $\mathcal{S}_{\Lambda}(U)>$ $-\infty$. Then the operator $\mathfrak{C}: \mathcal{P} / \mathcal{J}(\Lambda) \rightarrow R(\Lambda, U)$ is an isomorphism.

\section{$\S 8$. Construction of a special entire function}

In this section we construct an entire function $g \in \mathcal{W}(\Lambda)$ that does not expand in a series (1.2) if the groups $U_{m}$ approach one another asymptotically in a sufficiently strong way. We use the method exposed in Theorem 3.1 in [18].

Theorem 8.1. Let $\Lambda$ have finite upper density and be split into groups $U=\left\{U_{m}\right\}$ in such a way that $\mathcal{D}(\Lambda, U)<+\infty, \mathcal{S}_{\Lambda}(U)=-\infty$. Then there exists $g \in \mathcal{W}(\Lambda) \subset H(\mathbb{C})$ admitting no expansion (1.2) that converges uniformly on compact subsets of $\mathbb{C}$.

Proof. We have $\mathcal{S}_{\Lambda}(U)=-\infty$ by assumption. Consequently, there exist $\delta_{p} \in(0,1 / 6)$, $p \geq 1$, and a sequence $\left\{\lambda_{m(p), l(p)}\right\}$ such that

$$
\lim _{p \rightarrow \infty} \ln \left|q_{\Lambda, U}^{m(p), l(p)}\left(\lambda_{m(p), l(p)}, \delta_{p}\right)\right| /\left|\lambda_{m(p), l(p)}\right|=-\infty .
$$

Since $\mathcal{D}(\Lambda, U)<+\infty$, we may assume that

$$
\begin{aligned}
7\left|\lambda_{m(p), l(p)}\right| / 6 & \leq \min _{1 \leq l \leq M_{m(p+1)}}\left|\lambda_{m(p+1), l}\right|, \\
5\left|\lambda_{m(p+1), l(p+1)}\right| / 6 & \geq \max _{1 \leq l \leq M_{m(p)}}\left|\lambda_{m(p), l}\right|
\end{aligned}
$$

for all $p \geq 1$. Put $B(\alpha p)=B\left(\lambda_{m(p), l(p)}, \alpha \delta_{p}\left|\lambda_{m(p), l(p)}\right|\right)$ and

$$
g_{p}(z)=\frac{1}{2 \pi i} \int_{\partial B(5 p)} \frac{\exp (\lambda z) d \lambda}{\left(\lambda-\lambda_{m(p), l(p)}\right) q_{\Lambda, U}^{m(p), l(p)}\left(\lambda, \delta_{p}\right)}, \quad p \geq 1 .
$$

By the residue theorem, we obtain

$$
g_{p}(z)=b_{m(p), l(p)} \exp \left(\lambda_{m(p), l(p)} z\right)+\sum_{\lambda_{k} \in B(p) \backslash U_{m(p)}} b_{p, k} \exp \left(\lambda_{k} z\right),
$$

where $b_{m(p) l(p)}=\left(q_{\Lambda, U}^{m(p), l(p)}\left(\lambda_{m(p), l(p)}, \delta_{p}\right)\right)^{-1}$. Since $\left|q_{\Lambda, U}^{m(p), l(p)}\left(\lambda, \delta_{p}\right)\right| \geq 1, \lambda \in \partial B(5 p)$, we have

$$
\left|g_{p}(z)\right| \leq \sup _{\lambda \in \partial B(5 p)}|\exp (\lambda z)| \leq \exp \left(\operatorname{Re}\left(\lambda_{m(p), l(p)} z\right)+5 \delta_{p}\left|\lambda_{m(p), l(p)}\right||z|\right), \quad z \in \mathbb{C} .
$$

Consider the function

$$
g(z)=\sum_{p=1}^{\infty} c_{p} g_{p}(z), \quad c_{p}=q_{\Lambda, U}^{m(p), l(p)}\left(\lambda_{m(p), l(p)}, \delta_{p}\right), \quad p \geq 1
$$


We show that the series (8.4) converges uniformly on compact subsets in $\mathbb{C}$. Let $R>0$. By (8.1), there exists $p_{0}$ such that $\left|c_{p}\right| \leq \exp \left(-2 R\left|\lambda_{m(p), l(p)}\right|\right), p \geq p_{0}$. By (8.3) and (8.2), it follows that

$$
\sum_{p=1}^{\infty}\left|c_{p}\right| \max _{|z| \leq R}\left|g_{p}(z)\right| \leq A+\sum_{p=p_{0}}^{\infty} \exp \left(\left(-2 R+R+t \delta_{p} R\right)\left|\lambda_{m(p), l(p)}\right|\right)<+\infty .
$$

Thus, $g \in \mathcal{W}(\Lambda) \subset H(\mathbb{C})$. Suppose that $g$ is representable by the series $(1.2)$ that converges uniformly on compact subsets of $\mathbb{C}$. We have $N(\Lambda)<\infty$ by assumption. Therefore, as was mentioned in the Introduction, there exists a sequence $\left\{\mu_{k, n}\right\} \subset H^{*}(\mathbb{C})$ biorthogonal to $\mathcal{E}(\Lambda)$. Since the series (1.2) and (8.4) converge in the topology of $H(\mathbb{C})$, the coefficients of $z^{n} \exp \left(\lambda_{k} z\right)$ in the two series coincide and are equal to $\mu_{k, n}(g)$.

The convergence of the series (1.2) implies that

$$
\widetilde{g}_{m(p)}(z)=\sum_{\lambda_{k} \in U_{m(p)}} \sum_{n=0}^{n_{k}-1} d_{k, n} z^{n} \exp \left(\lambda_{k} z\right) \rightarrow 0, \quad p \rightarrow \infty, \quad z \in \mathbb{C} .
$$

Let $p \geq 1$. By (8.2) and the definition of $q_{\Lambda, U}^{m(p), l(p)}$, among all summands constituting $g_{s}$, $s \geq 1$, only one has an exponent belonging to the group $U_{m(p)}$. Therefore, $\tilde{g}_{m(p)}(z)=$ $c_{p} b_{m(p), l(p)} \exp \left(\lambda_{m(p), l(p)} z\right)$. By the above,

$$
c_{p} b_{m(p), l(p)} \exp \left(\lambda_{m(p), l(p)} z\right)=\exp \left(\lambda_{m(p), l(p)} z\right) \rightarrow 0, \quad p \rightarrow \infty, \quad z \in \mathbb{C},
$$

which is impossible. Thus, our assumption is false and the theorem is proved.

\section{§. BASIS IN AN INVARIANT SPACE OF ENTIRE FUNCTIONS}

In distinction with the case of a finite spectrum, the elements of the subspace $\mathcal{W}(\Lambda)$ with infinite spectrum are not necessarily representable as linear combinations of generalized eigenvectors of the differentiation operator on $\mathcal{W}(\Lambda)$, and even as series with respect to such vectors. As was mentioned in the Introduction, such a representation may by obstructed by a strong approach of the $\lambda_{k}$ to one another as $k \rightarrow \infty$. In this situation some elements of the system $\mathcal{E}(\Lambda)=\left\{z^{n} \exp \left(\lambda_{k} z\right)\right\}$ may be excessively similar (through still linearly independent). As a result, divergent series with respect to $\mathcal{E}(\Lambda)$ arise, which become convergent after some arrangement of brackets. Thus, some functions in $\mathcal{W}(\Lambda)$ (sums of "series with brackets") do not admit an $\mathcal{E}(\Lambda)$-series expansion. However, this can be remedied by replacing functions in $\mathcal{E}(\Lambda)$ with their linear combinations. For this, we need a procedure similar (but only in the essence) to orthogonalization of a complete system in a Hilbert space, resulting in a new system which is a basis. We are going to proceed with that.

Let $\Lambda$ be split into groups $U=\left\{U_{m}\right\}$. We denote by $Q(\Lambda, U)$ the projective limit of the Banach spaces

$$
Q_{p}(\Lambda, U)=\left\{\beta=\left\{\beta_{m, j}\right\}:\|\beta\|_{p}=\sup _{m, j}\left(\left|\beta_{m, j}\right| \exp \left(p\left|\lambda_{m, 1}\right|\right)\right)<\infty\right\}, \quad p \geq 1 .
$$

On the dual space $Q^{*}(\Lambda, U)$, we define an operator $\mathfrak{U}$ that takes any functional $\nu$ to the sequence $b=\left\{b_{n, l}\right\}=\left\{\nu\left(\beta^{n, l}\right)\right\}$, where $\beta^{n, l}=\left\{\beta_{m, j}\right\} \in Q(\Lambda, U)$ is such that $\beta_{n, l}=1$ and $\beta_{m, j}=0$ if $m \neq n$ and $j \neq l$. If $N(\Lambda)<\infty$ and $\mathcal{D}(\Lambda, U)<\infty$, then the series $\sum N_{m} \exp \left(-\varepsilon\left|\lambda_{m, 1}\right|\right)$ converges for every $\varepsilon>0$. Using this, it is easy to deduce (see [14, Lemma 7]) the following statement.

Lemma 9.1. Let $\Lambda$ have finite upper density and be split into groups $U=\left\{U_{m}\right\}$ in such a way that $\mathcal{D}(\Lambda, U)<\infty$. Then $Q(\Lambda, U)$ is reflexive and $\mathfrak{U}$ is an isomorphism between 
$Q^{*}(\Lambda, U)$ and $R(\Lambda, U)$. If $\nu \in Q^{*}(\Lambda, U)$, then

$$
\nu(\beta)=\sum_{m=1, j=1}^{\infty, N_{m}} \beta_{m, j} b_{m, j}, \quad \beta=\left\{\beta_{m, j}\right\} \in Q(\Lambda, U),
$$

where $b=\{b+m, j\}=\mathfrak{U}(\nu)$.

Let $f_{z}(\zeta)=\exp (z \zeta)$, and let $p_{m, j-1}\left(f_{z}\right)$ be defined by (7.4). We put

$$
\mathcal{E}(\Lambda, U)=\left\{e_{m, j}(z)\right\}, \quad e_{m, j}(z)=p_{m, j-1}\left(f_{z}\right), \quad m \geq 1, j=1, \ldots, N_{m} .
$$

For the first time, the system $\mathcal{E}(\Lambda, U)$ was introduced in [14. In that paper, some background for that was discussed, and a different representation of the functions $e_{m, j}(z)$ was given. The collection $\left\{e_{m, j}\right\}_{j=1}^{N_{m}}$ is defined as a special basis in the $N_{m}$-dimensional space generated by the functions $z^{n} \exp \left(\lambda_{k} z\right), n=0,1, \ldots, n_{k}-1, \lambda_{k} \in U_{m}$. The matrices of transition from $\mathcal{E}(\Lambda, U)$ to $\mathcal{E}(\Lambda)$ are defined explicitly. Note that if $N(\Lambda)<\infty$, then the system $\mathcal{E}(\Lambda, U)$ possesses a biorthogonal sequence of functionals, which are linear combinations of the terms of the sequence biorthogonal to $\mathcal{E}(\Lambda)$.

Our goal is to describe conditions under which every function $g \in \mathcal{W}(\Lambda)$ admits a unique series expansion

$$
g(z)=\sum_{m, j=1}^{\infty, N_{m}} \beta_{m, l} e_{m, j}(z)
$$

convergent uniformly on the compact subsets of $\mathbb{C}$. The existence of a biorthogonal sequence ensures the uniqueness of an expansion (9.1).

First, we study some properties of the system $\mathcal{E}(\Lambda, U)$. Let $K$ be a compact subset of $\mathbb{C}$ and take $s$ such that the closure of the disk $B(0, s)$ includes $K$. Then $\left\|f_{z}\right\|_{s} \leq 1$ for $z \in K$. By Lemma 7.1, there exist constants $C>0$ and $p \geq s$ such that

$$
\sup _{z \in K}\left|e_{m, j}(z)\right| \leq C \exp \left(p\left|\lambda_{m, 1}\right|\right), \quad m \geq 1, \quad j=1, \ldots, N_{m} .
$$

In this connection, we give the following definition (see [19]). A sequence $\left\{\widetilde{e}_{m, j}(z)\right\}$ of entire functions is said to be nearly exponential (with exponents $\lambda_{m, 1}$ ) if

1) for every $s \geq 1$ there exists $a>0$ and an index $p$ such that

$$
\sup _{|z| \leq s}\left|\widetilde{e}_{m, j}(z)\right| \leq a \exp \left(p\left|\lambda_{m, 1}\right|\right), \quad m \geq 1, \quad j=1, \ldots, N_{m} ;
$$

2) for every $s \geq 1$ there exists $b>0$ and an index $p$ such that

$$
b \exp \left(s\left|\lambda_{m, 1}\right|\right) \leq \sup _{|z| \leq p}\left|\widetilde{e}_{m, j}(z)\right|, \quad m \geq 1, j=1, \ldots, N_{m} .
$$

Properties (9.3) and (9.4) allow us to give a simple description of the space of coefficients of the series

$$
\sum_{m, j=1}^{\infty, N_{m}} \beta_{m, j} \widetilde{e}_{m, j}(z)
$$

that converge in the topology of $H(\mathbb{C})$, and then deduce theorems of Abel and CauchyHadamard type (see [20]) for these series. The following statement holds (see [19, Lemmas 1 and 3]).

Lemma 9.2. Let $\Lambda$ be split into groups $U=\left\{U_{m}\right\}$, and let $N(\Lambda), \mathcal{D}(\Lambda, U)<\infty$.

1) Suppose that (9.4) is fulfilled for the system $\left\{\widetilde{e}_{m, j}(z)\right\}$ and that the series (9.5) converges uniformly on compact subsets of $\mathbb{C}$. Then $\beta=\left\{\beta_{m, j}\right\} \in Q(\Lambda, U)$. 
2) Suppose that $\left\{\widetilde{e}_{m, j}(z)\right\}$ satisfies (9.3) and $\beta=\left\{\beta_{m, j}\right\} \in Q(\Lambda, U)$. Then for every $s \geq 1$ there exists constants $p$ and $A>0$ independent of $\beta$ and such that

$$
\sum_{m, j=1}^{\infty, N_{m}}\left|\beta_{m, j}\right| \sup _{|z| \leq s}\left|\widetilde{e}_{m, j}(z)\right| \leq A\|\beta\|_{p}
$$

In particular, the series (9.5) converges absolutely and uniformly on every compact subset of $\mathbb{C}$.

We say that $\left\{\widetilde{e}_{m, j}(z)\right\}$ is a Köthe basis in $\mathcal{W}(\Lambda)$ if every function $g \in \mathcal{W}(\Lambda)$ admits a unique series expansion (9.5) convergent uniformly on the compact subsets of $\mathbb{C}$, and for every $s \geq 1$ there exists $c>0$ and an index $p$ such that

$$
\sum_{m, j=1}^{\infty, N_{m}}\left|\beta_{m, j}\right| \sup _{|z| \leq s}\left|\widetilde{e}_{m, j}(z)\right| \leq c \sup _{|z| \leq p}|g(z)|, \quad g \in \mathcal{W}(\Lambda) .
$$

We mention yet another important property of the system $\left\{\widetilde{e}_{m, j}(z)\right\}$. We say that this system possesses the Köthe group property if for every $s \geq 1$ there exists an index $p$ and a constant $C>0$ such that for every $m \geq 1$ and $h_{m}(z)=\sum_{j=1}^{N_{m}} \alpha_{m, j} \widetilde{e}_{m, j}(z)$ we have

$$
\sum_{j=1}^{N_{m}}\left|\alpha_{m, j}\right| \sup _{|z| \leq s}\left|\widetilde{e}_{m, j}(z)\right| \leq C \sup _{|z| \leq p}\left|h_{m}(z)\right| .
$$

For a usual system of linearly independent vectors in a Euclidean space, such an inequality is always fulfilled. The value of $C$ depends on the minimal angle between these vectors.

We introduce an operator $\mathfrak{B}$ from $Q(\Lambda, U)$ to $\mathcal{W}(\Lambda)$ acting by the following rule: a sequence $\beta=\left\{\beta_{m, j}\right\} \in Q(\Lambda, U)$ is taken to the sum of the series (9.1) convergent uniformly on the compact subsets of $\mathbb{C}$.

Theorem 9.1. Let $U$ be a splitting of the sequence $\Lambda$ such that $\mathcal{S}_{\Lambda}(U)>-\infty$ and $\mathcal{N}(\Lambda, U), \mathcal{D}(\Lambda, U)<\infty$. Then

1) the operator $\mathfrak{B}: Q(\Lambda, U) \rightarrow \mathcal{W}(\Lambda)$ is an isomorphism;

2) $\mathcal{E}(\Lambda, U)$ is a Köthe bases in $\mathcal{W}(\Lambda)$;

3) $\mathcal{E}(\Lambda, U)$ is a nearly exponential sequence;

4) $\mathcal{E}(\Lambda, U)$ possesses the Köthe group property.

Proof. 1) By (9.2) and item 2) of Lemma 9.2, the operator $\mathfrak{B}$ is defined on the entire space $Q(\Lambda, U)$ and is continuous. By Lemma 2.2 , the quantity $M_{\Lambda}$ is finite and, with it, $N(\Lambda)$ is also finite by Lemma 2.1. Then an expansion of the form (9.1) is unique, and $\mathfrak{B}$ is an injection.

Every function $g \in \mathcal{W}(\Lambda)$ determines a continuous linear functional on the dual space $\mathcal{W}^{*}(\Lambda)$. Since $\mathcal{W}(\Lambda) \subset H(\mathbb{C})$ is a closed subspace of a Fréchet-Schwartz space, it is reflexive and $\mathcal{W}^{*}(\Lambda)$ is isomorphic to $H^{*}(\mathbb{C}) / \mathcal{W}^{0}(\Lambda)$, where $\mathcal{W}^{0}(\Lambda)$ is the set of functionals that annihilate $\mathcal{W}^{*}(\Lambda)$ (see [21]). Passing to Laplace transforms, we obtain isomorphism between $\mathcal{W}^{*}(\Lambda)$ and $\mathcal{P} / \mathcal{J}(\Lambda)$.

Consequently, there exists a continuous linear functional $\sigma$ on $\mathcal{P} / \mathcal{J}(\Lambda)$ such that $(\sigma,[f])=(g,[\mu]),[\mu] \in H^{*}(\mathbb{C}) / W^{0}(\Lambda)$, where $f$ is the Laplace transform of $\mu$. By Theorem 7.1, there exists $\rho \in R^{*}(\Lambda, U)$ such that

$$
(\rho, \mathfrak{C}([f]))=(\sigma,[f])=(g,[\mu]), \quad[\mu] \in H^{*}(\mathbb{C}) / W^{0}(\Lambda)
$$


By Lemma 9.1, the spaces $R^{*}(\Lambda, U)=\left(\mathcal{W}^{*}(\Lambda)\right)^{*}=\mathcal{W}(\Lambda)$ and $Q(\Lambda, U)$ are isomorphic, and there exists $\beta=\left\{\beta_{m, j}\right\} \in Q(\Lambda, U)$ such that

$$
(g,[\mu])=(\rho, \mathfrak{C}([f]))=\sum_{m=1, j=1}^{\infty, N_{m}} \beta_{m, j} p_{m, j-1}(f), \quad[\mu] \in H^{*}(\mathbb{C}) / \mathcal{W}^{0}(\Lambda) .
$$

For the role of the functional $\mu \in H^{*}(\mathbb{C})$, we take the $\delta$-function at the point $z \in \mathbb{C}$. Its Laplace transform is $f_{z}=\exp (z \zeta)$. By the definition of $e_{m, j}$ we have

$$
g(z)=\left(g,\left[\delta_{z}\right]\right)=\left(\rho, \mathfrak{C}\left(\left[f_{z}\right]\right)\right)=\sum_{m=1, j=1}^{\infty, N_{m}} \beta_{m, j} e_{m, j}(z), \quad z \in \mathbb{C} .
$$

By (9.2) and item 2) of Lemma 9.2, this series converges uniformly on the compact subsets of $\mathbb{C}$. This means that $\mathfrak{B}$ is a surjection and $\mathfrak{B}^{-1}(g)=\beta$. Since $\beta$ is constructed on the basis of isomorphism between $\mathcal{W}(\Lambda)$ and $Q(\Lambda, U)$, we see that $\mathfrak{B}^{-1}$ is continuous. Thus, 1) is true.

2) By item 1) and the uniqueness of a representation (9.1), the system $\mathcal{E}(\Lambda, U)$ is a basis in $\mathcal{W}(\Lambda)$. Next, by item 2) of Lemma 9.2, for every $s \geq 1$ there exists $p$ and a constant $A>0$ that do not depend on $\beta=\mathfrak{B}^{-1}(g)$ and are such that

$$
\sum_{m, j=1}^{\infty, N_{m}}\left|\beta_{m, j}\right| \sup _{|z| \leq s}\left|\widetilde{e}_{m, j}(z)\right| \leq A\|\beta\|_{p}
$$

Since $\mathfrak{B}^{-1}$ is continuous, there exist constants $l$ and $\widetilde{c}>0$ depending only on $p$ and such that $\|\beta\|_{p} \leq \widetilde{c} \sup _{|z|<l}|g(z)|$. Consequently, $\mathcal{E}(\Lambda, U)$ is a Köthe basis.

3) Formula (9.3) follows from (9.2). We prove (9.4). Suppose it is not true. Then there exists $s$ such that for every $p \geq 0$ there exists $m(p) \geq p$ and $j(p)$ with

$$
p^{-2} \exp \left(s\left|\lambda_{m(p), 1}\right|\right) \geq \sup _{|z| \leq p}\left|e_{m(p), j(p)}(z)\right| .
$$

Put $\beta_{m(p), j(p)}=\exp \left(-s\left|\lambda_{m(p), 1}\right|\right), p \geq 1$, and $\beta_{m, j}=0$ for $m \neq m(p)$ or $j \neq j(p)$. Then

$$
\begin{aligned}
\sup _{|z| \leq l}|g(z)| & =\sup _{|z| \leq l}\left|\sum_{m=1, j=1}^{\infty, N_{m}} \beta_{m, j} e_{m, j}(z)\right| \\
& =\sup _{|z| \leq l}\left|\sum_{p=1}^{\infty} \exp \left(-s\left|\lambda_{m(p), 1}\right|\right) e_{m(p), j(p)}(z)\right| \\
& \leq \sum_{p=1}^{\infty} \exp \left(-s\left|\lambda_{m(p), 1}\right|\right) \sup _{|z| \leq l}\left|e_{m(p), j(p)}(z)\right| \\
& \leq \sum_{p=1}^{l-1} \exp \left(-s\left|\lambda_{m(p), 1}\right|\right) \sup _{|z| \leq l}\left|e_{m(p), j(p)}(z)\right|+\sum_{p=l}^{\infty} p^{-2} .
\end{aligned}
$$

Thus, any function $g \in \mathcal{W}(\Lambda)$ expands in a series (9.1) convergent uniformly on the compact subsets of $\mathbb{C}$. Since this expansion is unique, by statement 1 ) we have $\beta=$ $\left\{\beta_{m, j}\right\} \in Q(\Lambda, U)$. But this is not true. Indeed,

$$
\|\beta\|_{n}=\sup _{m, j}\left(\left|\beta_{m, j}\right| \exp \left(n\left|\lambda_{m, 1}\right|\right)\right)=\sup _{p}\left(\exp \left((n-s)\left|\lambda_{m(p), 1}\right|\right)\right)=\infty, \quad n>s .
$$

Therefore, (9.4) is true.

Statement 4) is a direct consequence of 2). 
Remark. Statements 3) and 4) of Theorem 9.1 are also true in the case $\mathcal{S}_{\Lambda}(U)=-\infty$. In the proof of Statement 3), it suffices to impose yet another condition on $m(p)$, specifically,

$$
2 \max _{1 \leq j \leq N_{m(p)}}\left|\lambda_{m(p), j}\right| \leq \min _{1 \leq j \leq N_{m(p+1)}}\left|\lambda_{m(p+1), j}\right| .
$$

Then $\mathcal{S}_{\widetilde{\Lambda}}(\tilde{U})=0$, where $\widetilde{\Lambda} \cup U_{m(p)}$ and $\widetilde{U}=\left\{U_{m(p)}\right\}$. For $\widetilde{\Lambda}$ we can prove 1), 2), and then 3$)$. As a result, we arrive at a contradiction with (9.7). In a similar way, choosing an appropriate system $\widetilde{\Lambda}$, we can prove 4$)$.

Along with $\mathcal{E}(\Lambda, U)$, we consider other systems of functions. Put

$$
\tilde{e}_{m, j}(z)=\sum_{k=1}^{N_{m}} a_{m, j, k} e_{m, k}(z), \quad m \geq 1, j=1, \ldots, N_{m} .
$$

Let $\mathcal{E}(\Lambda, U, \mathbb{A})=\left\{\widetilde{e}_{m, j}\right\}$, let $\mathbb{A}=\left\{\mathcal{A}_{m}\right\}$, and let $\mathcal{A}_{m}=\left(a_{m, j, k}\right)$ be the matrix of transition from $\left\{e_{m, k}\right\}$ to $\left\{\widetilde{e}_{m, j}\right\}$. We say that $\mathcal{E}(\Lambda, U, \mathbb{A})$ is normed if $\max _{1 \leq k \leq N_{m}}\left|a_{m, j, k}\right|=1$, $j=1, \ldots, N_{m}, m \geq 1$.

Theorem 9.2. Suppose that a sequence $\Lambda$ with finite upper density is split into groups $U=\left\{U_{m}\right\}$ in such a way that $\mathcal{D}(\Lambda, U)<+\infty$. If $\mathcal{E}(\Lambda, U, \mathbb{A})$ is a basis in $\mathcal{W}(\Lambda)$, then $\mathcal{S}_{\Lambda}(U)>-\infty$.

Proof. By assumption, every function $g \in \mathcal{W}(\Lambda)$ admits a series expansion (9.5) and, consequently, an expansion (1.2) uniformly convergent on the compact sets. Consequently, $\mathcal{S}_{\Lambda}(U)>-\infty$ by Theorem 8.1 .

The following statements are immediate consequences of Theorem 9.1 and 9.2.

Theorem 9.3. Let a sequence $\Lambda$ with finite upper density be split into groups $U=\left\{U_{m}\right\}$ in such a way that $\mathcal{D}(\Lambda, U)<+\infty$. Suppose that $\mathcal{E}(\Lambda, U)$ is a basis in $\mathcal{W}(\Lambda)$. Then $\mathcal{E}(\Lambda, U)$ is a Köthe basis.

Theorem 9.4. Let a sequence $\Lambda$ with finite upper density be split into groups $U=\left\{U_{m}\right\}$ is such a way that $\mathcal{D}(\Lambda, U)<+\infty$. Suppose that the system $E(\Lambda, U, \mathbb{A})$ is a basis in $\mathcal{W}(\Lambda)$. Then $\mathcal{E}(\Lambda, U)$ is also a basis in $\mathcal{W}(\Lambda)$.

Theorem 9.5. Let a sequence $\Lambda$ with finite upper density be split into group $U=\left\{U_{m}\right\}$ in such a way that $\mathcal{D}(\Lambda, U)<+\infty$. Then the following statements are equivalent: 1$)$ $\mathcal{E}(\Lambda, U)$ is a basis in $\mathcal{W}(\Lambda) ; 2) \mathcal{S}_{\Lambda}(U)>-\infty$.

As a particular case, Theorem 9.5 involves the solution of the fundamental principle problem for an invariant subspace of entire functions (this solution was found in Theorem 5.1 of [6]).

Corollary. Let $\mathcal{W}(\Lambda)$ be nontrivial. Then the following statements are equivalent: 1) $\mathcal{E}(\Lambda)$ is a basis in $\mathcal{W}(\Lambda)$; 2) $\mathcal{S}_{\Lambda}>-\infty$.

Now, we describe the collection of all bases of $\mathcal{W}(\Lambda)$ constructed by a given splitting of $\Lambda$ into groups. First, we prove an auxiliary statement.

Lemma 9.3. Let a sequence $\Lambda$ be split into groups $U=\left\{U_{m}\right\}$, and let $N(\Lambda), \mathcal{D}(\Lambda, U)<$ $\infty, \mathcal{S}_{\Lambda}(U)>-\infty$. If $\mathcal{E}(\Lambda, U, \mathbb{A})$ is normed, then $\mathcal{E}(\Lambda, U, \mathbb{A})$ is a nearly exponential sequence.

Proof. We fix $s \geq 1$. By (9.2), there exist constants $p$ and $C>0$ such that

$$
\sup _{|z| \leq s}\left|e_{m, j}(z)\right| \leq C \exp \left(p\left|\lambda_{m, 1}\right|\right), \quad m \geq 1, \quad j=1, \ldots, N_{m} .
$$


Since $\mathcal{E}(\Lambda, U, \mathbb{A})$ is normed and $N(\Lambda), \mathfrak{D}(\Lambda, U)<\infty$, it follows that

$$
\sup _{|z| \leq s}\left|\widetilde{e}_{m, j}(z)\right| \leq \sum_{k=1}^{N_{m}} \sup _{|z| \leq s}\left|a_{m, j, k} e_{m, k}(z)\right| \leq N_{m} C \exp \left(p\left|\lambda_{m, 1}\right|\right) \leq a \exp \left((p+1)\left|\lambda_{m, 1}\right|\right) .
$$

This yields (9.3). Now, we prove (9.4). Let $s \geq 1$. By Theorem 9.1, inequality (9.4) is fulfilled for $\mathcal{E}(\Lambda, U)$. Therefore, there exist constants $p$ and $b>0$ such that

$$
b \exp \left(s\left|\lambda_{m, 1}\right|\right) \leq \sup _{|z| \leq p}\left|e_{m, k}(z)\right|, \quad m \geq 1, \quad k=1, \ldots, N_{m} .
$$

Moreover, $\mathcal{E}(\Lambda, U)$ possesses the Köthe group property. Thus, by (9.6), we have

$$
\sum_{k=1}^{N_{m}}\left|a_{m, j, k}\right| \sup _{|z| \leq p}\left|e_{m, k}(z)\right| \leq C \sup _{|z| \leq n}\left|\widetilde{e}_{m, j}(z)\right|, \quad m \geq 1, \quad j=1, \ldots, N_{m}
$$

where $n$ and $C>0$ depend only on $p$. Since $\mathcal{E}(\Lambda, U, \mathbb{A})$ is normed, for every $m \geq 1$ and $j=1, \ldots, N_{m}$ there exists a coefficient $a_{m, j, k}$ with unit modulus. By the above,

$$
b \exp \left(s\left|\lambda_{m, 1}\right|\right) \leq \min _{k} \sup _{|z| \leq p}\left|e_{m, k}(z)\right| \leq \sum_{k=1}^{N_{m}}\left|a_{m, j, k}\right| \sup _{|z| \leq p}\left|e_{m, k}(z)\right| \leq C \sup _{|z| \leq n}\left|\widetilde{e}_{m, j}(z)\right| .
$$

This yields the required estimate.

Let $\mathcal{E}(\Lambda, U, \mathbb{A})$ be such that the matrices $\mathcal{A}_{m}, m \geq 1$, are nonsingular, and let $\mathcal{B}_{m}=$ $\left(b_{m, j, k}\right)$ be the matrix inverse to $\mathcal{A}_{m}$. Put

$$
\mathfrak{a}(\mathbb{A})=\limsup _{m \rightarrow \infty} \max _{1 \leq j, k \leq N_{m}} \ln \left|b_{m, j, k}\right| /\left|\lambda_{m, 1}\right| .
$$

Let $\mathfrak{B}(\mathbb{A})$ be the operator taking any sequence $\beta=\left\{\beta_{m, j}\right\} \in Q(\Lambda, U)$ to the sum of the series $(9.5)$ (where $\left\{\widetilde{e}_{m, j}\right\}=\mathcal{E}(\Lambda, U, \mathbb{A})$ ), convergent in the topology of $H(\mathbb{C})$.

Theorem 9.6. Let $\Lambda$ be split into groups $U=\left\{U_{m}\right\}$, and let $N(\Lambda), \mathfrak{D}(\Lambda, U)<\infty$, $\mathcal{S}_{\Lambda}(U)>-\infty$. If the system $\mathcal{E}(\Lambda, U, \mathbb{A})$ is normed, then the following statement are equivalent:

1) $\mathcal{E}(\Lambda, U, \mathbb{A})$ is a basis in $\mathcal{W}(\Lambda)$

2) $\mathfrak{B}(\mathbb{A}): Q(\Lambda, U) \rightarrow \mathcal{W}(\Lambda)$ is an isomorphism;

3) $\mathcal{E}(\Lambda, U, \mathbb{A})$ is a Köthe basis in $\mathcal{W}(\Lambda)$;

4) $\mathcal{E}(\Lambda, U, \mathbb{A})$ possesses the Köthe group property;

5) $\mathfrak{a}(\mathbb{A})<\infty$.

Proof. 1) $\Longrightarrow 2$ ). By Lemma $9.3, \mathcal{E}(\Lambda, U, \mathbb{A})$ is a nearly exponential sequence. By $(9.4)$ and item 1) in Lemma 9.2, the operator $\mathfrak{B}(\mathbb{A})$ is a surjection. But by (9.3), item 2) in Lemma 9.2, and the uniqueness of an expansion with respect to the basis, the operator $\mathfrak{B}(\mathbb{A})$ is defined on the entire space $Q(\Lambda, U)$ and is injective. By the Banach inverse mapping theorem for Fréchet spaces, $\mathfrak{B}(\mathbb{A})$ is an isomorphism.

$2) \Longrightarrow 3$. By statement 2) and item 1) in Lemma $9.2, \mathcal{E}(\Lambda, U, \mathbb{A})$ is a basis in $\mathcal{W}(\Lambda)$. The inequality required for the Köthe basis property follows from the inequality in item 2 ) of Lemma 9.2 and the continuity of the operator inverse to $\mathfrak{B}(\mathbb{A})$.

The implication 3$) \Longrightarrow 4$ ) is obvious.

$4) \Longrightarrow 5)$. By (9.6), the identity $\sum \alpha_{m, j}(z) \equiv 0$ is impossible unless the $\alpha_{m, j}$ are all zero. Therefore, the matrices $\mathcal{A}_{m}\left(a_{m, j, k}\right), m \geq 1$, are nonsingular. By (9.6), for every $s \geq 1$ there exist constants $p$ and $C>0$ such that

$$
\sum_{k=1}^{N_{m}}\left|b_{m, j, k}\right| \sup _{|z| \leq s}\left|\widetilde{e}_{m, k}(z)\right| \leq C \sup _{|z| \leq p}\left|e_{m, j}(z)\right|, \quad m \geq 1, \quad j=1, \ldots, N_{m}
$$


It follows that

$\ln \left|b_{m, j, k}\right| \leq \ln \left(\sup _{|z| \leq p}\left|e_{m, j}(z)\right|\right)-\ln \left(\sup _{|z| \leq s}\left|\widetilde{e}_{m, k}(z)\right|\right)+\ln C, \quad m \geq 1, j, k=1, \ldots, N_{m}$.

By Lemma $9.3, \mathcal{E}(\Lambda, U)$ and $\mathcal{E}(\Lambda, U, \mathbb{A})$ are nearly exponential sequences. Thus, there exist $n, s$ and $a, b>0$ with

$$
\begin{aligned}
& b \exp \left(\left|\lambda_{m, 1}\right|\right) \leq \sup _{|z| \leq s}\left|\tilde{e}_{m, k}(z)\right|, \\
& \sup _{|z| \leq p}\left|e_{m, j}(z)\right| \leq a \exp \left(n\left|\lambda_{m, 1}\right|\right), \quad m \geq 1, \quad j, k=1, \ldots, N_{m} .
\end{aligned}
$$

Therefore, we have

$$
\ln \left|b_{m, j, k}\right| \leq n\left|\lambda_{m, 1}\right|-\left|\lambda_{m, 1}\right|+\ln a C-\ln b, \quad m \geq 1, \quad j, k=1, \ldots, N_{m} .
$$

Consequently, $\mathfrak{a}(\mathbb{A}) \leq n-1$.

$5) \Longrightarrow 1$ ). Let $g \in \mathcal{W}(\Lambda)$. If $g$ admits an expansion of the form (9.5) convergent uniformly on the compact subsets of $\mathbb{C}$, then this expansion is unique. Indeed, since the matrices $\mathcal{A}_{m}\left(a_{m, j, k}\right), m \geq 1$, are nonsingular, the system $\mathcal{E}(\Lambda, U, \mathbb{A}$ ) (along with $\mathcal{E}(\Lambda, U))$ possesses a biorthogonal sequence of functionals. By Theorem 9.1, the function $g$ expands in a series (9.1), and for every $n \geq 1$ we have

$$
\sum_{s=1, j=1}^{\infty, N_{m}}\left|\beta_{m, j}\right| \sup _{|z| \leq n}\left|e_{m, j}(z)\right|<\infty .
$$

By 5), there exists $l$ and a constant $B>0$ with

$$
\left|b_{m, j, k}\right| \leq B \exp \left(l\left|\lambda_{m, 1}\right|\right), \quad m \geq 1, \quad j, k=1, \ldots, N_{m} .
$$

Since $N(\Lambda), \mathcal{D}(\Lambda, U)<\infty$, we have $N_{m} \leq c \exp \left|\lambda_{m, 1}\right|, m \geq 1$. Taking (9.3) and (9.4) into account, we obtain

$$
\begin{aligned}
\sum_{m=1, j=1}^{\infty, N_{m}}\left|\beta_{m, j}\right| \sum_{k=1}^{N_{m}} \sup _{|z| \leq s}\left|b_{m, j, k} \widetilde{e}_{m, k}(z)\right| & \leq B a c \sum_{m=1, j=1}^{\infty, N_{m}}\left|\beta_{m, j}\right| \exp \left((l+p+1)\left|\lambda_{m, 1}\right|\right) \\
& \leq B a b^{-1} c \sum_{m=1, j=1}^{\infty, N_{m}}\left|\beta_{m, j}\right| \sup _{|z| \leq n}\left|e_{m, j}(z)\right|<\infty .
\end{aligned}
$$

Consequently,

$$
\begin{aligned}
\sum_{m=1, k=1}^{\infty, N_{m}} \tilde{e}_{m, k}(z) \sum_{j=1}^{N_{m}} \beta_{m, j} b_{m, j, k} & =\sum_{m=1, j=1}^{\infty, N_{m}} \beta_{m, j} \sum_{k=1}^{N_{m}} b_{m, j, k} \widetilde{e}_{m, k}(z) \\
& =\sum_{m=1, j=1}^{\infty, N_{m}} \beta_{m, j} e_{m, j}(z)=g(z),
\end{aligned}
$$

where the first series converges uniformly on the compact subsets of $\mathbb{C}$. This finishes the proof.

Theorem 9.6 yields a description of all possible bases in the subspace $\mathcal{W}(\Lambda)$ that consist of linear combinations of generalized eigenvectors of the differentiation operator formed inside the groups $U_{m}$ of exponents, under the condition that these groups are fairly well separated. By Theorem 9.2, there are no other bases of this type. Furthermore, all these bases are Köthe bases automatically. Next, the space of coefficients is completely described. By Theorem 2.1, required partitions always exist, moreover, their relative diameter can be made arbitrarily small. The following statement holds. 
Theorem 9.7. Let $\mathcal{W}(\Lambda)$ be a nontrivial invariant subspace in $H(\mathbb{C})$. For every $d>0$ there exists a partition $U$ of $\Lambda$ such that $\mathcal{D}(\Lambda, U)<d$ and the system $\mathcal{E}(\Lambda, U)$ is a basis in $\mathcal{W}(\Lambda)$.

The finiteness of the upper density (the nontriviality of $\mathcal{W}(\Lambda)$ ) may be insufficient for the existence of bases with respect to minimal partitions (i.e., partitions of zero relative diameter). An additional assumption is required. An ultimate case of such splitting (trivial splittings) was analyzed completely in the corollary to Theorem 9.2. In the general case, the situation is clarified with the help of Theorem 3.2.

Theorem 9.8. Let $\mathcal{W}(\Lambda)$ be a nontrivial invariant subspace in $H(\mathbb{C})$. The following statements are equivalent.

1) There exists a partition $U$ of $\Lambda$ into relatively small groups such that $\mathcal{E}(\Lambda, U)$ is a basis in $\mathcal{W}(\Lambda)$.

2) $S_{\Lambda}^{1}>-\infty$.

Consider also the situation where $\mathcal{W}(\Lambda)$ is the space of solutions on a convolution equation. The following theorem is a consequence of Theorem 9.8 and Corollary 2 to Theorem 6.1.

Theorem 9.9. Let $\mathcal{W}(\Lambda)$ be the space of solutions of a homogeneous convolution equation with characteristic function $f$. The following statements are equivalent.

1) There exists a partition $U$ of $\Lambda$ into relatively small groups such that $\mathcal{E}(\Lambda, U)$ is a basis in $\mathcal{W}(\Lambda)$.

2) $S_{\Lambda}^{1}>-\infty$.

3) There exists $a>0$ such that $\underline{h}_{f}(z) \geq a|z|, z \in \mathbb{C}$.

In conclusion, we present some more examples to elucidate the results.

Let $\Lambda=\left\{\lambda_{k}, n_{k}\right\}$, where $\lambda_{k-1}=k, \lambda_{2 k}=k+\xi_{k}, n_{k}=1, k \geq 1$, and $\ln \left|\xi_{k}\right| / k \rightarrow-\infty$ $k \rightarrow \infty$. The sequence $\Lambda$ has density 2. The last relation shows that $\mathcal{S}_{\Lambda}=-\infty$. By the corollary to Theorem 9.5 , the system $\mathcal{E}(\Lambda)$ is not a basis in $\mathcal{W}(\Lambda)$.

Consider the partition $U=\left\{U_{k}\right\}, U_{k}=\left\{\lambda_{2 k-1}, \lambda_{2 k}\right\}, k \geq 1$. Since $\lambda_{2 k+1}-\lambda_{2 k-1}=1$, arguing as in the example at the end of $\S 3$ we readily deduce that $\mathcal{S}_{\Lambda}(U)=0$. By Theorem 9.1, $\mathcal{E}(\Lambda, U)=\left\{e_{k, 1}, e_{k, 2}\right\}_{k=1}^{\infty}$ is a Köthe basis in $\mathcal{W}(\Lambda)$. By formulas (7.2) and (7.4), for $f(\zeta)=f_{z}(\zeta)=e^{\zeta z}$ we obtain

$$
e_{k, 1}(z)=e^{k z}, \quad e_{k, 2}(z)=\left(e^{\left(k+\xi_{k}\right) z}-e^{k z}\right) / \xi_{k}, \quad k \geq 1 .
$$

Now, let $n_{2 k-1}=2, n_{2 k}=1$, and let $\lambda_{k}, k \geq 1$, be the same as above. The relation $\mathcal{S}_{\Lambda}(U)=0$ is still fulfilled. By Theorem $9.1, \mathcal{E}(\Lambda, U)=\left\{e_{k, 1}, e_{k, 2}, e_{k, 3}\right\}$ is a Köthe basis in $\mathcal{W}(\Lambda)$. This partition is minimal (in the sense that the points $\lambda_{2 k-1}$ and $\lambda_{2 k}$ cannot be put in different groups) among all partitions with this property. Using (7.2) and (7.4) once again, it is easy to show that

$$
e_{k, 1}(z)=e^{k z}, \quad e_{k, 2}(z)=z e^{k z}, \quad e_{k, 3}(z)=2\left(e^{\left(k+\xi_{k}\right) z}-e^{k z}\left(1+\xi_{k}\right)\right) / \xi_{k}^{2}, \quad k \geq 1 .
$$

All other bases in $\mathcal{W}(\Lambda)$ with respect to the groups $U_{k}$ were described in Theorem 9.6. Let $\mathcal{E}(\Lambda, U, \mathbb{A})=\left\{\widetilde{e}_{k, 1}, \widetilde{e}_{k, 2}, \widetilde{e}_{k, 3}\right\}$, where

$$
\tilde{e}_{k, 1}(z)=e^{k z}, \quad \widetilde{e}_{k, 2}(z)=z e^{k z}, \quad \widetilde{e}_{k, 3}(z)=\left(e^{\left(k+\xi_{k}\right) z}-e^{k z}\right) / \xi_{k}, \quad k \geq 1 .
$$

If $\left|\xi_{k}\right| \leq 1, k \geq 1$, then the system $\mathcal{E}(\Lambda, U, \mathbb{A})$ is normalized. It can easily be shown that $\mathfrak{a}(\mathbb{A})=\lim _{k \rightarrow \infty}-\ln \left|\xi_{k}\right| / k=\infty$. Then by Theorem 9.6 the system $\mathcal{E}(\Lambda, U, \mathbb{A})$ is not a basis in $\mathcal{W}(\Lambda)$. This is related to the fact that the functions $\widetilde{e}_{k, 2}$ and $\widetilde{e}_{k, 3}$ behave quite similarly. If we replace $\widetilde{e}_{k, 3}$ with $\left(e^{\left(k+\xi_{k}\right) z}-e^{k z}\right) / \xi_{k}^{2}$, then the system ceases to be normalized; after normalization, we return to the system $\mathcal{E}(\Lambda, U, \mathbb{A})$. 


\section{REFERENCES}

[1] A. F. Leont'ev, Entire functions. Series of exponentials, Nauka, Moscow, 1983. (Russian) MR753827 $(86 \mathrm{j}: 30005)$

[2] V. V. Napalkov, Convolution equations in multidimensional spaces, Nauka, Moscow, 1982. (Russian) MR678923 (86g:46054)

[3] A. F. Leont'ev, Sequences of polynomials of exponentials, Nauka, Moscow, 1980. (Russian) $\operatorname{MR} 577300(81 \mathrm{~m}: 30002)$

[4] L. Schwartz, Théorie générale des fonctions moyenne-périodiques, Ann. of Math.(2) 48 (1947), no. 4, 857-929. MR0023948(9:428c)

[5] O. A. Gel'fond, Linear differential equations of infinite order with coefficients and asymptotic periods of entire functions, Tr. Mat. Inst. Steklova 38 (1951), 42-67. (Russian) MR0047776 (13:929a)

[6] A. S. Krivosheev, The fundamental principle for invariant subspaces in convex domains, Izv. Ross. Akad. Nauk Ser. Mat. 68 (2004), no. 2, 71-136; English transl., Izv. Math. 68 (2004), no. 2, 291-353. MR2058001 (2006g:30061)

[7] I. F. Krasichkov, Lower bound for entire functions of finite order, Sibirsk. Mat. Zh. 6 (1965), no. 4, 840-861. (Russian) MR0193236 (33:1457)

[8] A. S. Krivosheev and O. A. Krivosheeva, A basis in an invariant subspace of analytic functions, Mat. Sb. 204 (2013), no. 12, 49-104; English transl., Sb. Math. 204 (2013), no. 11-12, 1475-1796. MR.3185085

[9] B. Ya. Levin, Distribution of zeros of entire functions, Gosudarstv. Izdat. Tehn.-Teor. Lit., Moscow, 1956. MR0087740 (19:402c) English transl., Amer. Math. Soc., Providence, RI, 1964. MR0156975 $(28: 217)$

[10] P. Lelong and L. Gruman, Entire functions of several complex variables, Grundlehren Math. Wiss., Bd. 282, Springer-Verlag, Berlin, 1986. MR837659 (87j:32001)

[11] V. S. Azarin, The indicators of an entire function and the regularity of the growth of the Fourier coefficients of the logarithm of its modulus, Funktsional. Anal. i Prilozhen. 9 (1975), no. 1, 47-48; English transl., Funct. Anal. Appl. 9 (1975), no. 1, 41-42. MR0367202 (51:3444)

[12] A. S. Krivosheev and V. V. Napalkov, Complex analysis and convolution operators, Uspekhi Mat. Nauk 47 (1992), no. 6, 3-58; English transl., Russian Math. Surveys 47 (1992), no. 6, 1-56. MR.1209144(94e:32003)

[13] A. S. Krivosheev, Indicators of entire functions and the continuation of solutions of a homogeneous convolution equation, Mat. Sb. 184 (1993), no. 8, 81-108; English transl., Sb. Math. 79 (1994), no. 2, 401-423. MR1239760 (94k:32002)

[14] _ Basis by "relatively small clusters", Ufim. Mat. Zh. 2 (2010), no. 2, 67-69. (Russian)

[15] _ An almost exponential sequence of exponential polynomials, Ufim. Mat. Zh. 4 (2012), no. 1, 88-106; English transl., Ufa Math. J. 4 (2012), no. 1, 82-100.

[16] L. Hörmander, The analysis of linear partial differential operators. I. Distribution theory and Fourier analysis, Grundlehren Math. Wiss., Bd. 256, Springer-Verlag, Berlin, 1983. MR717035 (85g:35002a)

[17] L. I. Ronkin, Introduction to the theory of entire functions of several variables, Nauka, Moscow, 1971. MR0320357 (47:8896) English transl., Transl. Math. Monogr., vol. 44, Amer. Math. Soc., Providence, RI, 1974. MR0346175 (49:10901)

[18] O. A. Krivosheeva, Singular points of the sum of series of exponential monomials on the boundary of the convergence domain, Algebra i Analiz 23 (2011), no. 2, 162-205; English transl., St. Petersburg Math. J. 23 (2012), no. 2, 321-350. MR2841675 (2012h:30006)

[19] A. S. Krivosheev, An almost exponential basis, Ufim. Mat. Zh. 2 (2010), no. 1, 87-96. (Russian)

[20] O. A. Krivosheeva, Convergence domain for series of exponential polynomials, Ufim. Mat. Zh. 5 (2013), no. 4, 84-90; English transl., Ufa Math. J. 5 (2013), no. 4, 82-87.

[21] A. Grothendieck, Sur les espaces $(F)$ et $(D F)$, Summa Brasil. Math. 3 (1954), 57-123. MR0075542 $(17: 765 \mathrm{~b})$

Institute of Mathematics With Computer Centrum, Russian Academy of Sciences, ul. CherNYSHEVSKOGO 112, 450048 Ufa, Russia

Baskhir State University, ul. Zaki Validi 32, 450076 Ufa, Russia

E-mail address: kriolesya2006@yandex.ru

Received 5/FEB/2014 